\title{
LOW-FREQUENCY-INDUCED SYNAPTIC POTENTIATION: A PARADIGM SHIFT IN THE \\ FIELD OF MEMORY-RELATED PLASTICITY MECHANISMS?
}

by

Diala Sleiman Habib

A thesis submitted to the Centre for Neuroscience Studies

In conformity with the requirements for

The degree of Doctor of Philosophy

Queen's University

Kingston, Ontario, Canada

(October, 2010)

Copyright $@$ Diala Sleiman Habib, 2010 


\begin{abstract}
It is assumed that plasticity involving up-and down regulation of synaptic strength (i.e., long-term potentiation, LTP; long-term depression, LTD) mediates learning and memory processes. Typically, high-frequency stimulation (HFS) of afferent fibers results in LTP, while lowfrequency stimulation (LFS) elicits LTD. In stark contrast to this "HFS- LTP vs. LFS-LTD" dogma, the present thesis characterizes a novel form of LFS-induced LTP in the septohippocampal system. The first set of experiments show that alternating, single pulse stimulation $(1 \mathrm{~Hz})$ of the medial septum (MS) and CA3 hippocampal $(\mathrm{H})$ commissural fibres results in a long-lasting potentiation of field excitatory postsynaptic potentials (fEPSPs) in CA1 of urethane-anesthetised rats (MS-H-LTP). MS-H LTP is long lasting ( $>5 \mathrm{~h})$, requires a specific inter-stimulus interval of $1 \mathrm{~s}$ between MS and CA3 stimulation, saturates with repeated stimulation episodes and depends on NMDA receptor activation. In the third chapter (review) I suggest that LFS protocols may more accurately mimic some oscillatory activity patterns $(\sim 1 \mathrm{~Hz})$ present in hippocampal and neocortical circuits during sleep-related memory consolidation. Moreover, I compare the mechanisms underlying classical, HFS-LTP to those mediating MS-H LTP as well as several other types of LFS-LTP in the hippocampus and amygdala in vitro. Subsequently, I investigated cellular mechanisms of MS-H LTP and their similarity to classical HFS-LTP via drug application at the CA1 recording site and showed that MS-H LTP depends on protein kinase A and protein synthesis. This surprising similarity between mechanisms mediating HFS-LTP and MS-H LTP was further supported by occlusion experiments whereby LFS and HFS, delivered to the same animal, competed for the available synaptic potentiation of CA3-CA1 synapses. The final experiments showed that MS-H LTP is compromised in early aged rats, while similar levels of potentiation are expressed in the juvenile and adult hippocampus. Interestingly,
\end{abstract}


MS-H LTP could not be induced (i.e., was occluded) $3 \mathrm{~h}$ after training on the hidden platform version of the Morris water maze, while it was unaltered at 8 and $24 \mathrm{~h}$ intervals. This thesis characterizes a novel form of hippocampal plasticity at the cellular, synaptic and behavioural level and suggests that LFS-LTP may mediate processes of sleep-related memory consolidation. 


\section{Co-Authorship}

In all cases, I (Diala Habib) participated in the study design, data acquisition, data analysis, and manuscript preparation. Hans C. Dringenberg contributed to the study design, data analysis, and manuscript preparation.

Chapter 2 has been published in its entirety, and can be cited as:

Habib, D and Dringenberg, H.C. (2009) Alternating Low-Frequency Stimulation of Medial Septal and Commissural Fibers Induces NMDA-Dependent, Long-Lasting Potentiation of Hippocampal Synapses in Urethane-Anesthetized Rats. Hippocampus 19: 299-307.

Chapter 3 has been published in its entirety, and can be cited as:

Habib, D and Dringenberg, H.C. (2009) Low-frequency induced synaptic potentiation: A paradigm shift in the field of memory-related plasticity mechanisms? Hippocampus 20: 29-35.

Chapter $\mathbf{4}$ has been published in its entirety, and can be cited as:

Habib, D and Dringenberg, H.C. Surprising similarity between mechanisms mediating low (1 $\mathrm{Hz})$ - and high-frequency $(100 \mathrm{~Hz})$ - induced, long-lasting synaptic potentiation in CA1 of the intact hippocampus. Neuroscience 170: 489-496. 


\section{Acknowledgements}

First and foremost, I would like to thank my supervisor Dr. Hans Dringenberg for his tremendous support and guidance these past five years. My first meeting with Hans was a few weeks after I had already accepted Queen's offer into their graduate program and what started off as instinctdriven decision ended up being the best one I could have made for my career. Hans is a talented scientist with an infinite amount of patience for his students, especially for those of us who in the beginning stages didn't have the slightest clue about what we were doing, not even enough to develop a poster for their first conference presentation. I hope to lead by his example one day, as a scientist and mentor, and I will consider myself to be successful if I eventually become even a quarter of the scientist he is today. Hans, I sincerely thank you for all that you have taught me and you can rest assure that I will still pick up the phone and call when I won't be able to make sense of my data, which inevitably is bound to happen in my career.

I would like to take this opportunity to thank my thesis advisory committee Dr. Reynolds, Dr. Rose and Dr. Beninger for all their guidance, support and constructive feedback throughout my graduate studies. Also, I would like to thank Lisa Miller-Willberforce for getting me over my fear of rats and more importantly for all her guidance and supervision on animal care these past five years. I could have not done it without you Lisa!

I would like to thank my colleagues in the Dringenberg lab: Min-Ching, for teaching me all the technical skills I know today, Jenny, for our refreshing conversations in the lab that often times had nothing to do with science, Peter, for his encouragement and passion for hippocampal electrophysiology ha!, Audrey, for reminding me of the importance of remaining calm when experiments aren't working well and last but not least, Claudia for her infinite enthusiasm and positive attitude.

Thank you to the rest of my colleagues on the fourth floor, Scott Hayton for his enlighten scientific ideas, Jonathan Beuk and Tomek Bananiskowski for their wonderful sense of humor and their ability to always make me laugh. I wish you all the best of luck in finishing your doctoral degrees. 
To my dear friends Alisha Hemraj, Niousha Ghazni, Emily Hawkin, Apostolia Petropoulou, Megan Mahoney, Danielle Malone and Jordan Letich, I want to thank you for being such a strong support system while I was here in Kingston. You all are like my sisters away from home and I will forever remember our wonderful times together, I love you all very much!

To my wonderful husband-to-be, Ramzy, I want to thank you for your continuous support and emotional stability, especially these past two years. You have been immensely patient with me during this chaotic time of my life. Thank you elbi from the bottom of heart.

I want to thank my uncles Simon and George and my aunt Afaf for being the older brothers and sister I never had, and for encouraging me to continuously move forward in my education. Uncle Hassan, I would like to especially thank you for your continuous support and encouragement, I love you for all that you have done for me, and I only hope you go after your own dreams with as much intensity as you pushed me to go after mine.

I would like to take this opportunity to thank my brothers Anthony and Basil for all the laughter, love and support these past five years. I love you both very much, and I hope that your futures are bright and filled with success and happiness!

I dedicate this thesis to my mother, Nada, and my father, Sleiman Habib. Thank you so much for your love, support and dedication throughout my life. You continuously encouraged me to try to be the best I can be and I would not be where I am today if it was not for the both of you. 


\section{Table of Contents}

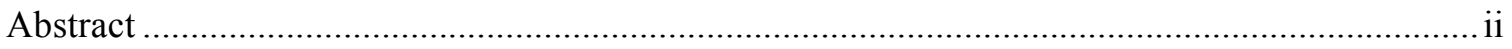

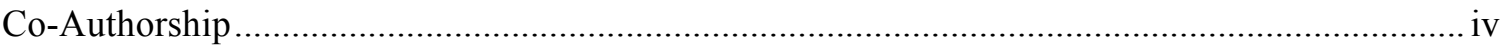

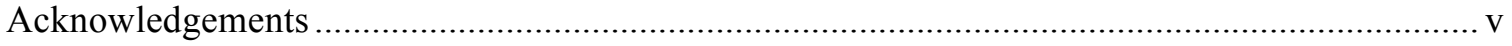

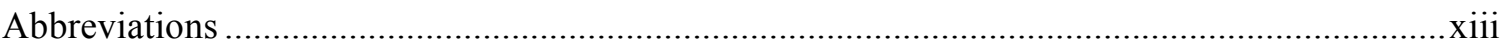

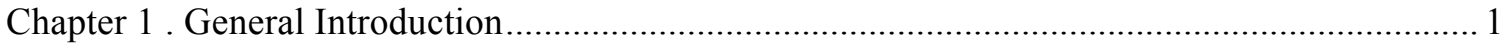

1.1 Long-term potentiation (LTP): Induction protocols and mechanisms ................................ 1

1.2 Common induction protocols used to elicit LTP in the hippocampus ................................ 2

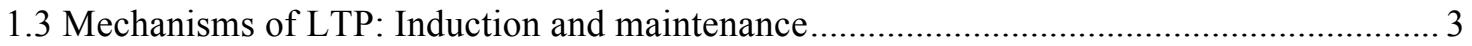

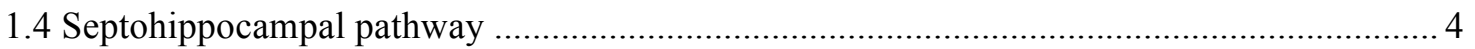

1.4.1 Projections from medial septum (MS) to hippocampus................................................ 4

1.4.2 Modulation of LTP by the septohippocampal pathway ................................................. 6

1.4.3 Role of the septohippocampal system in spatial memory ............................................. 8

1.4.4 The septohippocampal system in the aged brain: Behavioural and LTP studies ............ 9

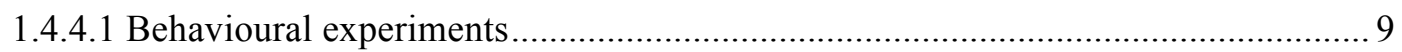

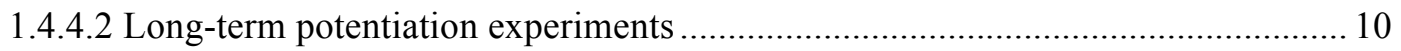

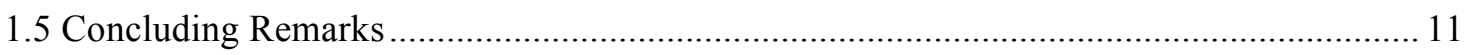

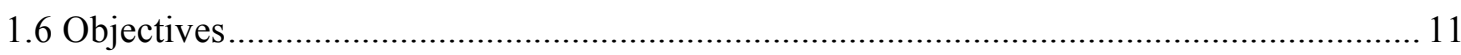

Chapter 2. Alternating low-frequency stimulation of medial septum and hippocampal fibers induces a long-lasting synaptic potentiation of hippocampal synapses (MS-H LTP) ................... 13

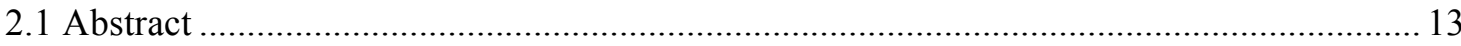

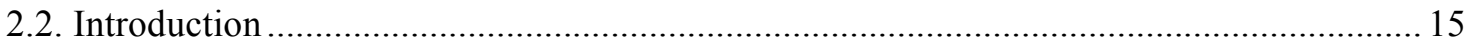

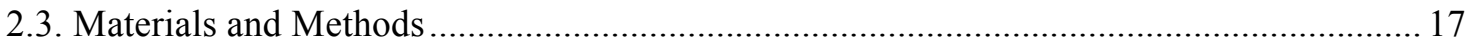

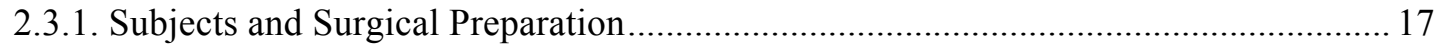

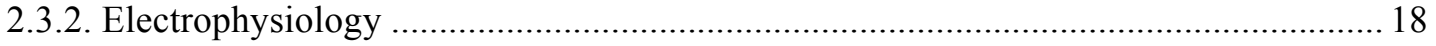

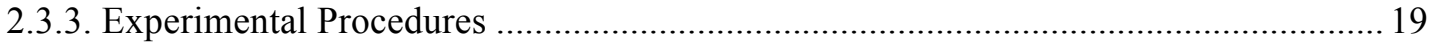

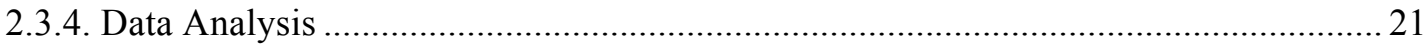

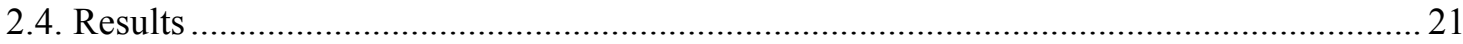

2.4.1. Alternating single pulse stimulation of the MS and CA3 elicits an increase in fEPSP

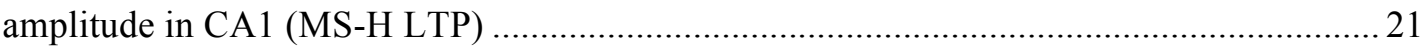

2.4.2. MS-H LTP is dependent on NMDA, but not cholinergic receptor activation ............. 29 
2.4.3. Enhancement of fEPSP amplitude is associated with decreases in PPF ratio 34

2.5. Discussion

Chapter 3 . Low and high frequency induced synaptic potentiation: A comparative review of induction protocols, mechanisms and implications. 42

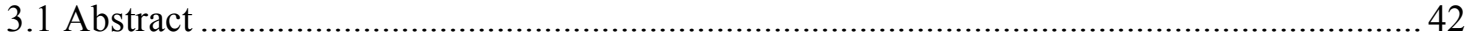

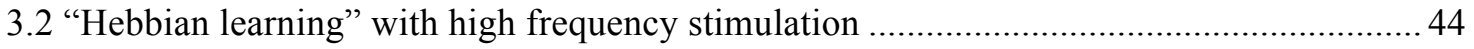

3.3 High frequency induced LTP mechanisms: The general consensus ................................... 44

3.4 From high to low: Have HFS protocols met their match? .................................................. 45

3.5 HFS-LTP vs. LFS-LTP mechanisms: How similar are they? ............................................ 48

3.6 LFS-LTP: Relevance to endogenous hippocampal activity .............................................. 50

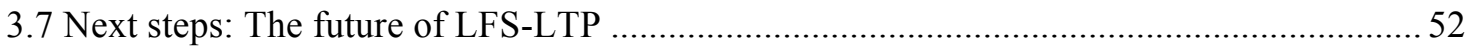

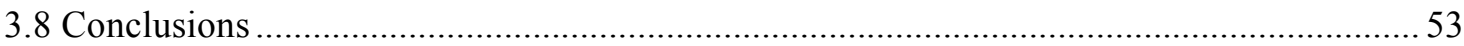

Chapter 4 . Surprising similarity between mechanisms mediating low $(1 \mathrm{~Hz})$-and high $(100 \mathrm{~Hz})-$ induced long-lasting synaptic potentiation in CA1 of the intact hippocampus ..............................55

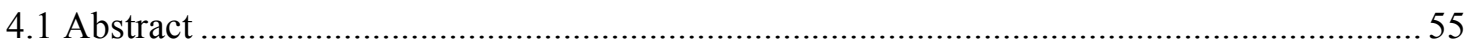

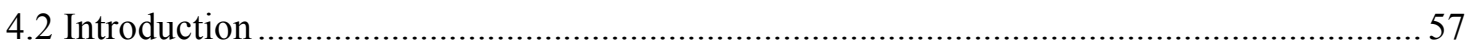

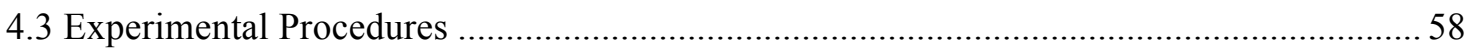

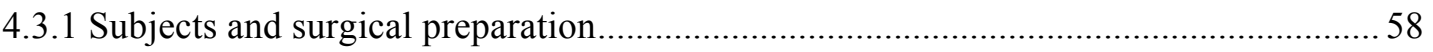

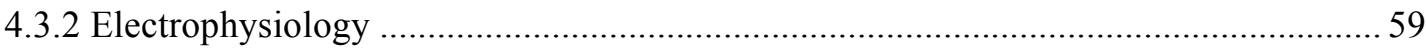

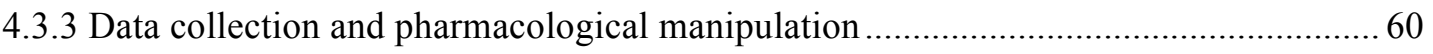

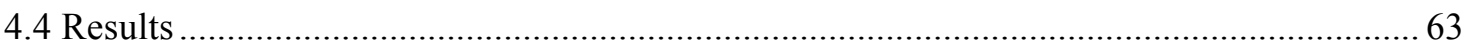

4.4.1 Synaptic enhancement following alternating MS-CA3 single pulse stimulation (MS-H

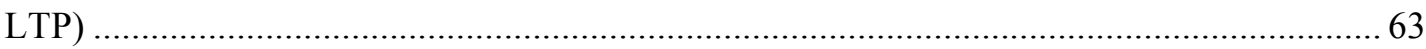

4.4.2 MS-H LTP is dependent on protein synthesis and PKA activation ............................... 63

4.4.3 Competition between LTP induced by LFS $(1 \mathrm{~Hz})$ and HFS $(100 \mathrm{~Hz})$........................ 70

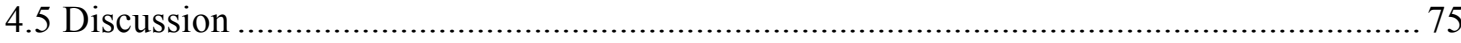

Chapter 5 . Influence of age and behavioural experience on the expression of MS-H LTP........ 79

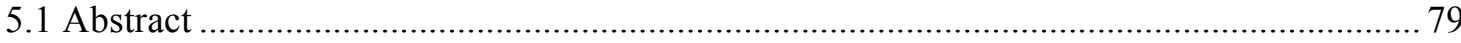

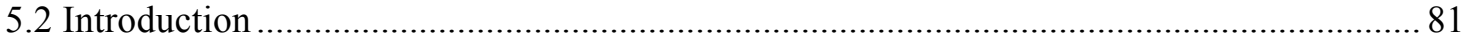

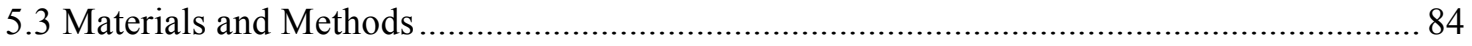

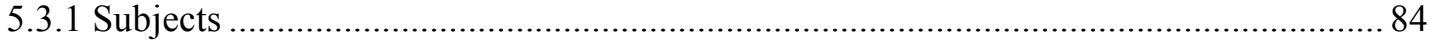

5.3.2 Experiment 1: Influence of age on expression of MS-H LTP .................................... 84

viii 


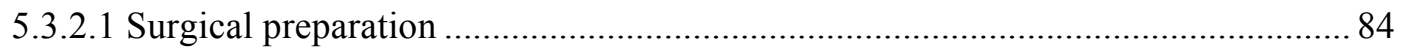

5.3.2.2 Electrophysiology and Experimental Procedures ................................................. 85

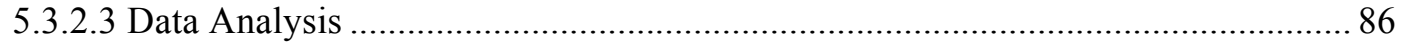

5.3.3 Experiment 2: Influence of behavioral experience (water maze training) on expression

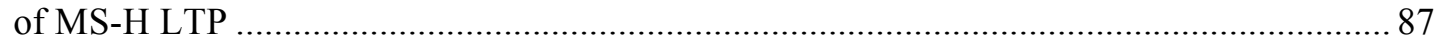

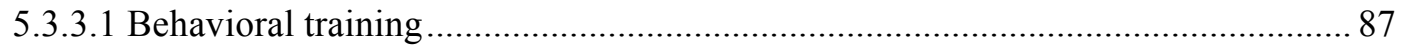

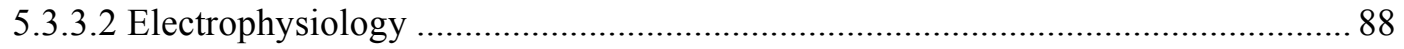

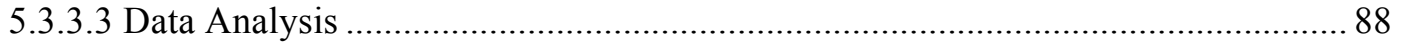

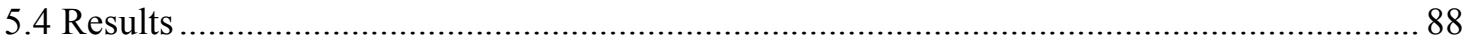

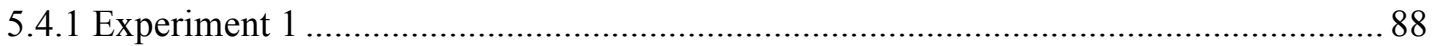

5.4.1.1 MS-H LTP in the juvenile (27-33 days), adult (3-4 months) and early aged (14

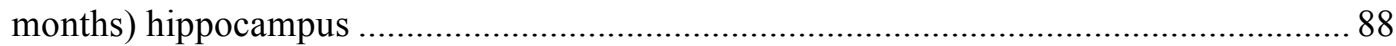

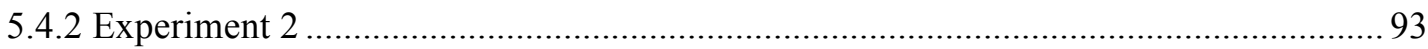

5.4.2.1 Effects of water maze training on MS-H LTP .......................................................... 93

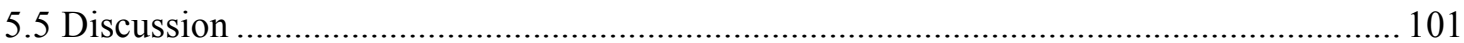

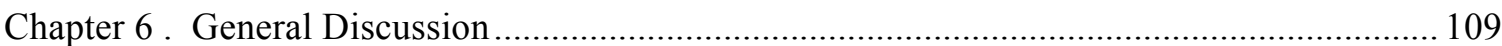

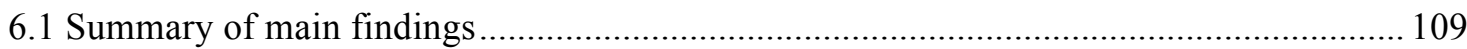

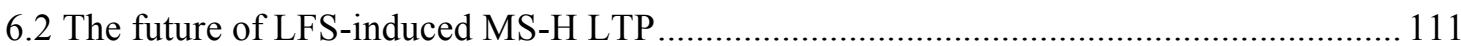

6.2.1 Can MS-H LTP be expressed in freely moving animals? ......................................... 111

6.2.2 Do activity patterns similar to the MS-CA3 induction protocol occur endogenously in

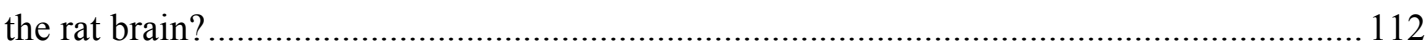

6.2.3 Do alternating LFS patterns of $1 \mathrm{~Hz}$ effectively induce LFS-LTP in areas outside the

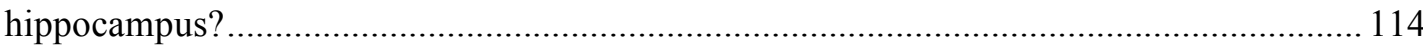

6.2.4 Do forms of LFS-LTP mediate early consolidation of striatal-dependent learning tasks?

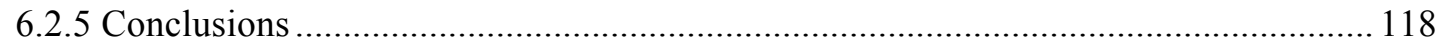

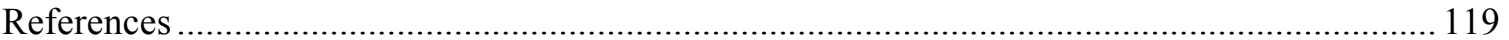




\section{List of Figures}

Figure 1. Effects of alternating medial septum (MS)-CA3 stimulation on amplitude of field excitatory postsynaptic potentials (fEPSPs) recorded in CA1 of urethane-anaesthetized rats. ..... 24

Figure 2. The effects of various interstimulus intervals (ISIs) between alternating stimulation of the medial septum (MS) and CA3 area on amplitude of CA1 field excitatory postsynaptic potentials (fEPSPs) averaged between 1.5 and $2 \mathrm{~h}$ following the alternating stimulation.

Figure 3. The effects of multiple induction episodes of alternating stimulation of the medial septum (MS) and CA3 area on amplitude of CA1 field excitatory postsynaptic potentials (fEPSPs)

Figure 4. Effect of MK-801 and AP-V treatment on changes in field excitatory postsynaptic potential (fEPSP) amplitude following alternating medial septum (MS)-CA3 stimulation.

Figure 5. The effects of scopolamine and mecamylamine on enhancement in field excitatory postsynaptic potential (fEPSP) amplitude in CA1 following medial septum (MS)-CA3 alternating stimulation.

Figure 6. Changes in paired pulse facilitation (PPF) ratio and field excitatory postsynaptic potential (fEPSP) amplitude in CA1 following medial septum (MS)-CA3 alternating stimulation.

Figure 7. The effect of alternating stimulation of the medial septum (MS) and CA3 area on amplitude of CA1 field excitatory postsynaptic potentials (fEPSPs) in the stratum radiatum..... 65

Figure 8. Local application of anisomycin or H89 prevents enhancement of field excitatory postsynaptic potential (fEPSP) amplitude following alternating medial septum (MS)-CA3

stimulation. 68

Figure 9. Competition between LTP induced by alternating medial septum (MS)-CA3 stimulation and high frequency stimulation (HFS) of CA3-CA1 synapses. .72 
Figure 10. The effects of alternating medial septum (MS)-CA3 stimulation on amplitude of CA1 field excitatory postsynaptic potentials (fEPSPs) in 27-33 day (juvenile), 3-4 month (adult) and 14-month-old (early aged) rats.

Figure 11. Average escape latency across 12 trails for rats trained on the hidden $(n=20)$ and visible platform $(n=7)$ version of the Morris water maze task.

Figure 12. Effects of water maze training on MS-H LTP in the urethane-anesthetized preparation. .96

Figure 13. Input/output curves of rats exposed to water maze training. 100 


\section{List of Tables}

Table 1. Summary of mechanisms underlying low frequency stimulation (LFS)-induced longlasting synaptic potentiation in the hippocampus and amygdala. 


\section{Abbreviations}

\begin{tabular}{|c|c|}
\hline $\mathrm{aCSF}$ & artificial cerebrospinal fluid \\
\hline AMPA & amino-3-hydroxy-5-methyl-4-isoxazolepropionic acid \\
\hline ANOVA & analysis of variance \\
\hline BLA & basolateral amygdala \\
\hline $\mathrm{Ca}^{2+}$ & calcium \\
\hline CaMKII & calcium -calmodulin-dependent protein kinase II \\
\hline cAMP & cyclic adenosine monophosphate \\
\hline CREB & cAMP response element binding protein \\
\hline DB & diagonal band of Broca \\
\hline DMSO & dimethyl sulfoxide \\
\hline EPSP & excitatory postsynaptic potential \\
\hline fEPSP & field excitatory postsynaptic potential \\
\hline $\mathrm{H}$ & hippocampal \\
\hline HFS & high-frequency stimulation \\
\hline i.p. & intraperitoneal \\
\hline ISI & interstimulus interval \\
\hline LFS & low-frequency stimulation \\
\hline LTD & long-term depression \\
\hline LTP & long-term potentiation \\
\hline MWM & Morris water maze \\
\hline MS & medial septum \\
\hline NMDA & N-methyl-D-aspartate \\
\hline
\end{tabular}




$\begin{array}{ll}\text { NMDAR } & \text { N-methyl-D-aspartate receptor } \\ \text { PKA } & \text { protein kinase A } \\ \text { PKC } & \text { protein kinase C } \\ \text { RNA } & \text { ribonucleic acid } \\ \text { S.E.M. } & \text { standard error of the mean } \\ \text { SH } & \text { septohippocampal } \\ \text { TBS } & \text { theta burst stimulation } \\ \text { VLGUT } & \text { vesicular glutamate transporter }\end{array}$




\section{Chapter 1. General Introduction}

\subsection{Long-term potentiation (LTP): Induction protocols and mechanisms}

In the past several decades, there has been considerable evidence to suggest that mechanisms mediating learning and memory involve activity-dependent changes in synaptic connectivity. The guiding principle for such synaptic modifications was initially proposed by Donald Hebb (1949) in his book, The Organization of Behaviour. According to Hebb's visionary postulate, the co-activation of a pre- and postsynaptic cell will ultimately increase the strength of the connection between the two neurons.

Currently, long-term potentiation (LTP), a long-lasting increase in strength of synapses, typically elicited by high frequency stimulation (HFS) of afferent fibers, is one of the many experimental models used to study the synaptic modifications that underlie learning and memory. Typical forms of LTP induced by a brief burst of HFS exhibit key properties initially described by Hebb's postulate, that the co-activation of pre- and postsynaptic neurons is responsible for the induction of synaptic changes that take place during information storage (Hebb, 1949). Moreover, LTP exhibits common cellular and molecular properties to those mechanisms known to mediate learning and memory consolidation (e.g., protein synthesis dependency and long lasting maintenance). Consequently, extensive research in the hippocampus and extra-hippocampal systems has validated LTP as a model for studying the synaptic modifications that underlie acquisition of information and memory encoding in various experimental preparations. 


\subsection{Common induction protocols used to elicit LTP in the hippocampus}

The initial LTP study (Bliss and Lómo, 1973) characterized a long-lasting synaptic potentiation in the dentate gyrus in response to HFS to afferent perforant path fibers $(15 \mathrm{~Hz}$ stimulation for $15-20 \mathrm{~s}$, or $100 \mathrm{~Hz}$ train for 3-4 s), with $100 \mathrm{~Hz}$ stimulation trains being most effective in eliciting this phenomenon. Consequently, this pattern of stimulation continues to be among the most frequently utilized induction protocols for eliciting synaptic potentiation in hippocampal and extrahippocampal systems (Raymond, 2007).

Currently, there exist several variations of the first LTP induction protocol used by Bliss and Lómo (1973), all typically involving high frequency trains of stimulation (100 Hz, tetanization) that reliably induce LTP in the hippocampal formation. For example, short (20-40 ms) trains of stimulation at $200-400 \mathrm{~Hz}$, generally separated by a few seconds are commonly used to induce LTP at perforant path-dentate gyrus synapses (Raymond, 2007). A common induction protocol used to induce long-lasting synaptic potentiation in CA1 pyramidal cells also consists of a brief burst of $100 \mathrm{~Hz}$ stimulation for 1 second (HFS) that can be repeated up to 6 times, with more bursts resulting in greater LTP in vivo (Raymond, 2007). Remarkably, repeated HFS trains (up to 50 times) have been shown to induce synaptic potentiation in the dentate gyrus that persists for at least one year from the last induction episode in freely moving animal models (Abraham et al., 2002).

Since the initial discovery of LTP in the hippocampus, researchers have attempted to establish induction protocols that closely resemble endogenous neuronal firing patterns of neurons or afferent fibers to the hippocampal formation. For example, Stäubli and Lynch (1987) identified a pattern of stimulation consisting of brief bursts of HFS, which are repeated at $5 \mathrm{~Hz}$, mimicking the endogenous theta rhythms $(3-12 \mathrm{~Hz})$ present in the hippocampus during specific 
types of behavior (e.g., walking, postural adjustments; Vanderwolf, 1969, 1988; Buzsáki, 2002). Collectively, these commonly used HFS protocols are reliable in the induction of LTP in the hippocampus and have been instrumental in deciphering mechanisms mediating the induction, consolidation and maintenance of long-lasting synaptic enhancement.

\subsection{Mechanisms of LTP: Induction and maintenance}

Commonly, LTP induced by HFS can be divided into distinct temporal processes, with an initial, rapid induction phase (seconds to minutes) which is followed by a delayed, long lasting stabilization and maintenance phase ( $>1$ hour).

Induction Phase: The cellular and molecular mechanisms underlying long-lasting synaptic potentiation have been characterized with great detail in several neural networks. The mechanisms mediating "classical" HFS-induced LTP in CA1 pyramidal cells, in particular, have received significant attention due to its critical role in specific forms of hippocampal-dependent learning (e.g., spatial learning, declarative memory; Jarrard, 1993; Eichenbaum, 2006). The induction of long lasting synaptic enhancement at CA3-CA1 synapses elicited by HFS of presynaptic axons is dependent on initial activation of postsynaptic amino-3-hydroxy-5-methyl-4isoxazolepropionic acid (AMPA), metabrotropic, and N-methyl-D-aspartate receptors (NMDARs; Bliss and Collingridge, 1993; Malenka and Nicoll, 1999; Riedel et al., 2003). The removal of the voltage-dependent magnesium block of NMDARs upon depolarization of the membrane subsequently allows calcium $\left(\mathrm{Ca}^{2+}\right)$ entry into dendritic spines, resulting in a rise in

intracellular $\mathrm{Ca}^{2+}$, consequent activation of $\mathrm{Ca}^{2+}$-calmodulin-dependent protein kinase II (CaMKII) and activity of protein kinase C (PKC; Lynch et al., 1983; Nicoll and Malenka, 1999).

Maintenance Phase: The initiation of the maintenance phase of hippocampal LTP relies on intracellular events that lead to the phosphorylation and re-distribution of AMPA receptors 
and activity of cAMP response element binding protein (CREB), that ultimately result in mRNA transcription and subsequent protein synthesis (Roberson et al. 1999; Kandel and Squire, 2000; Ahmed and Frey, 2005; Miyamoto, 2006). These mechanisms are thought to ultimately lead to structural remodeling, subsequent growth of new dendritic spines and enlargement of pre-existing spines (Luscher et al. 2000; Malenka and Bear, 2004). The maintenance phase of LTP has also been suggested to not only depend on postsynaptic mechanisms, but may involve presynaptic contributions as well. While evidence for these putative presynaptic mechanisms is lacking in vivo, evidence in vitro suggests that a retrograde messenger is released from the postsynaptic dendrite and diffuses back across the synapse to reach the presynaptic axon terminal (see Medina and Izquierdo, 1995). Here, this retrograde signal appears to result in an increased level of $\mathrm{Ca}^{2+}$ and PKC activation, which ultimately result in an increased probability of vesicle release from the presynaptic terminal (Kleschevnikov and Routtenberg, 2001). Some of the candidate retrograde messengers in the hippocampus include archidonic acid (Williams et al., 1989), nitric oxide, and carbon monoxide (Williams et al., 1989; Böhme et al., 1991). However, the lack of data supporting the precise contributions of presynaptic mechanisms in vivo has lead to an unclear consensus regarding their role in the maintenance of synaptic plasticity in the mammalian brain.

\subsection{Septohippocampal pathway}

\subsubsection{Projections from medial septum (MS) to hippocampus}

The mechanisms mediating hippocampal synaptic plasticity are modulated by heterosynaptic afferent fibers emanating from specific cortical and subcortical regions, such as the basal forebrain area (Jay et al., 1996; Hasselmo, 1999). For example, a major source of hippocampal input originates from ascending fibers located in the medial septum (MS) and the 
adjacent vertical limb of the diagonal band of Broca (DB), together making up the septohippocampal (SH) pathway (Köhler et al., 1984; Amaral and Kurz, 1985). The septal neurons innervate the hippocampus through three focal routes that includes the fimbria, dorsal fornix and the supracallosal striae, while a fourth ventral septal route passes through the amygdala complex and terminates primarily in the subuculum (Milner and Amaral, 1984; Gage et al., 1984; Dutar et al., 1995). The MS contains diverse neuronal populations and, thus, supplies a heterogeneous connectivity to the hippocampal formation including parallel cholinergic, GABAergic and glutamatergic fibers (Dudar, 1977; Dutar et al., 1995; Sotty et al., 2003). The majority of cholinergic cells make synaptic connections primarily with dendritic spines of excitatory and inhibitory neurons in the CA3, CA1 and dentate gyrus of the hippocampus (Houser et al., 1983; Wainer et al., 1984; Léránth and Frotscher, 1987). In contrast, GABAergic projections seem to exhibit a higher degree of target cell specificity and mainly terminate on distinct populations of GABAergic nonpyramidal neurons (Freund and Antal, 1988). Moreover, the hippocampus provides a monosynaptic projection back to the MS/DB that originates primarily from GABAergic neurons of the stratum oriens of CA1/CA3 area and synapses preferentially on GABAergic and, to a lesser extent, on cholinergic neurons (Tóth et al., 1993). It appears that the majority of the anatomical work of the SH pathway typically focused on GABA and acetylcholine because, until very recently, the existence of non-cholinergic/non-GABAergic septal neurons had not been investigated.

Interestingly, more recent electrophysiological and anatomical evidence raises the possibility that SH projections may also provide the hippocampus with glutamatergic transmission inputs (Sotty et al., 2003; Colom et al., 2005; Bland et al., 2007). Evidence from multiplex reverse transcription-polymerase chain reactions have characterized a subpopulation of 
SH neurons that express mRNAs for vesicular glutamate transporters (VGLUT1 and VGLUT2), presumed to be contained in neurons that have the capacity to release glutamate (Danik et al., 2003; Sotty et al., 2003). Additional work using an intact preparation of the septum in vitro showed that glutamatergic neurons in the MS/DB can generate excitatory input to local cholinergic and GABAergic neurons in the presence of cholinergic agonists (Manseau et al., 2005). While, these highly novel findings suggest that the glutamatergic SH projections modulate endogenous hippocampal activity, at present the role of this putitative projection system requires further investigation. Collectively, the data summarized here clearly demonstrate the heterogeneity of the SH pathway, and extensive work has shown the importance of these projections in modulating plasticity at hippocampal synapses.

\subsubsection{Modulation of LTP by the septohippocampal pathway}

Several experimental studies have provided evidence to suggest that inputs from the MS modulate LTP and excitability in the hippocampus in vitro and in intact brain preparations (Krnjević and Ropert, 1982; Huerta and Lisman, 1993; Leung et al., 2003; Ovsepian et al., 2004; Ovsepian, 2006; Dringenberg et al., 2008). While all three major fiber systems contribute to the cellular activity and plasticity of hippocampal neurons, currently, the majority of studies has focused on the influence of cholinergic afferent fibers on the facilitation of hippocampal LTP, effects that can be mediated by muscarinic or nicotinic receptor mechanisms (Markram and Segal, 1990; Huerta and Lisman, 1993; Auerbach and Segal, 1994; Fujii et al.,1999; Matsuyama et al., 2000; Leung et al., 2003; Ovsepian et al., 2004; Ji et al., 2001; Dringenberg et al., 2008).

Interestingly, studies on hippocampal neurons in vitro have shown that acetylcholine exerts its effects on synaptic facilitation in part by modulating hippocampal oscillatory theta activity, rhythmic patterns that are prominent during waking/exploratory behaviours (Huerta and 
Lisman, 1993). In fact, some evidence suggests that in the presence of cholinergic-induced hippocampal theta activity, changes in synaptic plasticity are more prominent under these conditions such that a HFS is no longer required for LTP induction (Huerta and Lisman, 1993). It appears that the modulatory influence of acetylcholine on endogenous hippocampal rhythms is stronger during walking behaviours compared to awake immobility, slow-wave and rapid-eye movement sleep in freely moving rodents (Hasselmo, 1999; Leung et al., 2003). These and similar investigations have provided support for the notion that hippocampal LTP is enhanced in the presence of elevated acetylcholine release, a form of facilitation apparently related to specific behavioral states.

The GABAergic fibers that parallel these prominent cholinergic projections have also been shown to influence hippocampal excitability. For example, electrical stimulation of the MS markedly enhances CA1 population spikes evoked by Schaffer collateral/commissural stimulation through a disinhibitory, GABAergic mechanism (Ovsepian, 2006). These indirect influences mediated by GABAergic disinhibition may provide an additional mechanism by which the MS modulates hippocampal LTP and other forms of plasticity.

While the majority of studies have examined the modulatory effects of acetylcholine and to some degree GABAergic influences on hippocampal LTP, this does not exclude a possible contribution of glutamatergic afferent fibers on to the facilitation of hippocampal plasticity. For example, there is some evidence to suggest that HFS of the MS elicits a long-lasting synaptic facilitation in CA1, an effect abolished by NMDAR antagonists in urethane-anesthetized rats (Markevich et al., 1997). Thus, it appears that these glutamatergic fiber projections from the MS may contribute to mechanisms of hippocampal LTP and thus, merit further investigation. 
Nonetheless, the data summarized here clearly demonstrate a vital role of heterosynaptic medial septal afferent fibers on mechanisms in the modulation of plasticity in the hippocampus.

\subsubsection{Role of the septohippocampal system in spatial memory}

Several reports have confirmed a pivotal role of the hippocampal formation in learning and memory that is partly dependent on afferent fibers originating in the medial septal area (Gray and McNaughton, 1983; Squire, 1992; Dutar et al., 1995). The earlier lines of evidence supporting the role of the hippocampus in spatial memory showed that selective hippocampal lesions disrupt animals' performance in the Morris water maze (MWM), a well established test of spatial orientation and memory (Morris, 1981). In this task, a rat is placed in a circular pool filled with opaque water containing a hidden escape platform just below the surface of the water in a fixed location. Visual cues, such as colored objects, placed around the pool in plain sight of the animal allow it to form an association of the fixed visual cues with the hidden escape platform (spatial memory). Animals with ventral and dorsal hippocampal lesions display longer latencies in locating the hidden escape platform, demonstrating deficits in spatial memory that are not present in control non-lesioned subjects (Morris et al., 1982).

These deficits in performance caused by lesions to hippocampal circuitry were later shown to be partly due to a disruption of cholinergic input from the MS, as animals with selective lesions of cholinergic cell bodies in the MS display MWM deficits that are similar to those observed with direct hippocampal damage (Kelsey and Landry, 1988; Riekkinen et al., 1990). Interestingly, rats with cholinergic medial septal lesions improve their escape latency with training, while their initial heading errors upon entering the maze, a proposed index of accuracy in their spatial map, remains compromised (Sutherland et al., 1983; Kelsey and Landry, 1988). Further, introducing a visual cue that marks the location of the hidden escape platform (i.e., visual 
platform version of MWM task), a procedure that minimizes the need for spatial memory, completely eliminates performance deficits in medial septal lesioned rats (Kelsey and Landry, 1988). Thus, it appears that SH cholinergic projections facilitate the formation of a spatial map (reference memory) that represents the location of the platform with respect to the distal cues surrounding the pool. The role of these cholinergic inputs in spatial learning has been further demonstrated by evidence showing that septal cholinergic transplants restore cholinergic function and spatial learning abilities in SH lesioned rats (Dunnett et al., 1982; Low et al., 1982; Segal et al., 1989). It is noteworthy, however, to mention that fimbria/fornix lesions disrupting both afferent and efferent fibers to the hippocampus also affect non-cholinergic innervation such as GABA and glutamatergic transmission. However, the contribution of these non-cholinergic inputs from the MS to the hippocampus in spatial memory is not well established. Nevertheless, the series of data summarized above demonstrate the importance of the SH system in learning and spatial memory and moreover, is well studied in the aged mammalian brain.

\subsubsection{The septohippocampal system in the aged brain: Behavioural and LTP studies}

\subsubsection{Behavioural experiments}

It is well known that aged rats are consistently impaired in learning the location of the hidden escape platform, a deficit in spatial memory that is not often observed in the visible platform version of the MWM task (Frick et al., 1995; Rosenzweig et al., 1997; Shen et al., 1997). It appears that this age-related behavioural deficit is similar to the impairments observed after septal fimbria/fornix lesions in young rats (Bresnahan, 1992) suggesting that the SH system may contribute to age-related decline in spatial memory. Moreover, reports have shown that deficits in spatial memory are correlated with the age-related decreases in the number and size of 
cholinergic neurons in the MS (Fischer et al., 1989; 1992; Nilsson and Gage, 1993). Conversely, intrahippocampal implantation of cells grafted from the septal region of fetal rats, rich in cholinergic neurons, has been shown to reverse age-related spatial memory decline (Gage and Björklund, 1986). Correlations between age-related changes in neural systems and deficits in spatial memory have also been extended to glutamatergic dysfunction and deficits in hippocampal NMDAR activity (Magnusson, 1998). However, whether or not NMDAR dysfunction in the aged hippocampus is partly due to deficits in glutamatergic or other noncholinergic input from the MS is currently unknown. On the other hand, these data summarized above are consistent with the view that impaired function in the cholinergic system and its projections to the hippocampus contributes to age-related deficits in learning and memory.

\subsubsection{Long-term potentiation experiments}

The age-related physiological changes that occur in the hippocampal circuitry not only contribute to cognitive decline as previously mentioned, but also appear to underlie age-related deficits in the induction and maintenance of hippocampal LTP (Rosenzweig and Barnes, 2003; see also Bergado and Almaguer, 2002). Several reports have shown that aged animals show deficits in NMDAR-dependent LTP induction using robust, HFS protocols (e.g., 100-200 Hz) at CA3-CA1 synapses in vitro (Deupree et al., 1993; Rosenzweig et al., 1997). Specifically, aged animals appear to show a rapid decline in LTP and reduced depolarization of neurons in CA1, deficits that may in part be due to changes in baseline levels of membrane excitability, calcium regulation and/or overall loss of synapses in the hippocampal network (Deupree et al., 1993;

Rosenzweig et al., 1997; Rosensweig and Barnes, 2003). Further, several reports have shown a rapid decay in LTP in aged rats relative to adults in vitro and in intact brain preparations (Barnes, 1979; Barnes and McNaughton. 1980; Bach et al., 1999). These data suggest that aging also 
appears to affect protein synthesis-dependent mechanisms mediating the maintenance of longlasting synaptic potentiation. Interestingly, these age-dependent changes in synaptic potentiation correlated with age-related impairments in learning and spatial memory (Barnes and McNaughton, 1985; Rosenzweig et al., 1997).

In summary, the MS exerts modulatory influences on hippocampal plasticity as seen at the behavioural, synaptic and cellular level in adult animals. It appears that physiological changes in the aging hippocampus involve modifications in these afferent fiber projections, and may contribute to age-related impairments in spatial memory and deficits in hippocampal synaptic potentiation.

\subsection{Concluding Remarks}

Here I have provided a brief overview of the common induction protocols used to elicit LTP in the hippocampal formation. I describe the well-established cellular and molecular mechanism mediating LTP and discuss the modulatory influence of the MS on hippocampal plasticity. Evidently, the SH system plays a role in spatial memory and age-related modifications in this system may contribute to the cognitive decline and corresponding deficits of hippocampal synaptic potentiation seen in the aged mammalian brain.

\subsection{Objectives}

The majority of experimental protocols use patterns of HFS to elicit classical LTP in both hippocampal and extra-hippocampal systems. In contrast, protocols employing LFS $(<5 \mathrm{~Hz})$ typically result in minimal LTP or in long-term depression (LTD) of hippocampal synapses (Bramham and Srebro, 1987; Christie and Abraham, 1992; Christie et al., 1994; Thiels et al., 1994; Massey and Bashir, 2007). The overall objective of this thesis was to challenge this long- 
standing dogma of "HFS-LTP vs. LFS-LTD” using combined in vivo electrophysiology and behavioural studies.

The main objective of the first empirical paper (chapter 2) was to determine whether LFS $(\sim 1 \mathrm{~Hz})$ of the MS and CA3 hippocampal (H) fibers can elicit long-lasting synaptic potentiation in CA1 in urethane anesthetised rats. These initial series of experiments led to the discovery and characterization of LFS-induced MS-H LTP. Next, I wrote a review paper (Chapter 3) with the objective to discuss the potential functional significance of LFS-LTP in sleep-related memory consolidation. In addition I compared the well-known synaptic and cellular mechanisms underlying classical, high-frequency-induced LTP to those mediating the more recentlydiscovered phenomena of LFS-LTP in the hippocampus and amygdala. Subsequently, in my second empirical paper (chapter 4) my main objective was to investigate the overlap in cellular mechanisms of MS-H LTP and their similarity to classical HFS-LTP by means of in vivo microdialysis and occlusion experiments. Finally, the last objective of this thesis (chapter 5) was to examine age-dependent changes in MS-H LTP, as well as to decipher the behavioural significance of this novel form of high-frequency independent, long-lasting synaptic potentiation. 


\title{
Chapter 2. Alternating low-frequency stimulation of medial septum and hippocampal fibers induces a long-lasting synaptic potentiation of hippocampal synapses (MS-H LTP)
}

\author{
As published in "Alternating Low-Frequency Stimulation of Medial Septal and Commissural \\ Fibers Induces NMDA-Dependent, Long-Lasting Potentiation of Hippocampal Synapses in \\ Urethane-Anesthetized Rats" by Habib, D \& Dringenberg, HC. 2009. Hippocampus, Vol. 19, \\ 299-307.
}

\subsection{Abstract}

Recent evidence indicates that some synapses exhibit long-lasting synaptic potentiation in response to low frequency $(1 \mathrm{~Hz})$ stimulation, similar to long-term potentiation (LTP) following high-frequency induction protocols. Here, we characterize a form of long-lasting synaptic potentiation in the hippocampal CA1 area following alternating, single pulse stimulation of the medial septum (MS) and hippocampal CA3 commissural fibers (MS-H LTP). In urethaneanesthetized rats, alternating single pulse stimulation of the MS and CA3 (50 pulses each at 0.5 $\mathrm{Hz}, 1000 \mathrm{~ms}$ interstimulus interval [ISI]) produced gradual increases of field excitatory postsynaptic potential (fEPSP) amplitude in CA1 ( $123 \%$ of baseline), while MS or CA3 stimulation alone was ineffective. The fEPSP enhancement was long-lasting $(>4 \mathrm{~h})$ and repeated 
episodes of alternating MS-CA3 stimulation tended to result in greater levels of potentiation than those elicited by a single episode. Surprisingly, ISIs of 500, 750, and $1500 \mathrm{~ms}$ did not result in significant changes in fEPSP amplitude, while an ISI of $100 \mathrm{~ms}$ produced synaptic depression. MS-H LTP was resistant to systemic administration of nicotinic and muscarinic receptor antagonists (scopolamine, mecamylamine), but abolished by systemic MK-801 (0.5 mg/kg, i.p.) or local CA1 application of AP-V (10 mM), indicative of a critical role of hippocampal NMDA receptors in this effect. Paired-pulse facilitation experiments revealed a gradually developing, significant inverse correlation between fEPSP enhancement and decrease in paired pulse facilitation ratio, suggesting a role of changes in presynaptic transmitter release. Together, these data demonstrate a novel form of long-lasting synaptic enhancement in CA1 neurons in response to low frequency activity in separate afferent systems, activity which might mimic some aspects of natural discharge patterns during the acquisition or consolidation of memory processes in hippocampal circuits. 


\subsection{Introduction}

The hippocampus receives diffuse fiber inputs from basal forebrain areas that have been shown to contribute to learning and memory processes (Hasselmo, 1999; Hasselmo and Giocomo, 2006). The input to the hippocampal formation from the medial septum (MS) is comprised of at least three distinct, parallel neurochemical pathways, consisting of cholinergic, GABAergic and glutamatergic fibers (Dudar, 1977; Dutar et al., 1995; Sotty et al., 2003). While all three fiber systems likely contribute to cellular activity and plasticity of hippocampal neurons, to date, the majority of work has examined the influence of cholinergic and GABAergic inputs to the hippocampus. Cholinergic fibers from the MS to the hippocampus are known to facilitate excitability and long-lasting plasticity (e.g., long-term potentiation, LTP) induction at hippocampal synapses in vitro and in vivo (Krnjević and Ropert, 1982; Markram and Segal, 1990; Huerta and Lisman, 1993; Auerbach and Segal, 1994; Leung et al., 2003; Ovsepian et al., 2004; Dringenberg et al., 2008), effects that can be mediated by both muscarinic (Huerta and Lisman, 1993; Leung et al., 2003; Ovsepian et al., 2004; Dringenberg et al., 2008) or nicotinic receptor mechanisms (Fujii et al., 1999; Matsuyama et al., 2000; Ji et al., 2001). These and similar investigations have provided support for the notion that hippocampal plasticity/LTP is enhanced in the presence of elevated acetylcholine release.

GABAergic inputs from the MS to the hippocampal formation have also been shown to modulate hippocampal excitability. For example, electrical stimulation of the MS markedly enhances CA1 population spikes evoked by Schaffer collateral/commissural stimulation through a disinhibitory, GABAergic mechanism (Krnjević et al., 1988; Ovsepian, 2006). These indirect 
influences on pyramidal cell excitability could provide an additional mechanism to modulate long-lasting plasticity at hippocampal synapses.

More recently, electrophysiological and anatomical studies have raised the possibility that septohippocampal projections might also release glutamate as a transmitter substance in the hippocampus (Sotty et al., 2003; Colom et al., 2005; Bland et al., 2007). At present, the role of this putative projection system in the regulation of hippocampal activity has not been examined. Nevertheless, the data summarized above clearly support the notion that different hippocampal afferents originating in the MS play an important role in modulating activity and plasticity processes at hippocampal synapses.

The majority of studies examining hippocampal plasticity and its modulation by extrahippocampal systems have used typical LTP protocols that require high frequency- or theta burst stimulation of glutamatergic afferents to induce long-lasting changes in synaptic efficacy (e.g., Larson et al., 1986; Larson and Lynch, 1988; Huerta and Lisman, 1993; Schulz et al., 1994; Leung et al., 2003; Ovsepian et al., 2004). In contrast, lower frequency stimulation (LFS) produces minimal or no LTP, or may result in long-term depression (LTD) (Bramham and Srebro, 1987; Christie and Abraham, 1992; Christie et al., 1994; Thiels et al., 1994; Wasling et al., 2002; Massey and Bashir, 2007). However, recent evidence indicates that some synapses can show long-lasting synaptic potentiation in response to LFS (1 Hz). Lanté et al. (2006a, b) showed that, in hippocampal slices, $1 \mathrm{~Hz}$ stimulation of the CA1 area produces a slow-onset synaptic potentiation in close proximity to the stimulation site, an effect that was not observed in CA3. Similarly, Huang and Kandel (2007) demonstrated a slow-onset, protein synthesis-dependent potentiation of synaptic efficacy at cortico-amygdala synapses following $1 \mathrm{~Hz}$ stimulation, which required co-activation of glutamatergic and neuromodulatory (serotonergic, dopaminergic, and 
noradrenergic) signals. Collectively, these data suggest that, in specific fiber systems, the initiation of LTP-like processes can occur in the absence of high-frequency activation of glutamatergic synapses.

At present, the extent to which lower frequency inputs are effective in inducing synaptic potentiation in different forebrain systems, and the contributions of neuromodulatory signals to such forms of plasticity, are not well understood. Here, we characterize a form of long-lasting hippocampal synaptic plasticity in the CA1 area following alternating, single pulse stimulation $(0.5 \mathrm{~Hz})$ of the MS and Schaffer collateral/commissural pathway in vivo (MS-H LTP).

Surprisingly, MS-H LTP is resistant to both muscarinic and nicotinic receptor antagonists, but is abolished by hippocampal NMDA receptor blockade. These experiments demonstrate that longlasting synaptic enhancement can be induced by repeated, infrequent co-activation of separate afferent systems to the hippocampal CA1 area in vivo.

\subsection{Materials and Methods}

\subsubsection{Subjects and Surgical Preparation}

All experiments were performed on male Long-Evans rats (320-450 g) housed in a colony room (12/12-h reverse light cycle) with food and water available ad libitum. The procedures were conducted in accordance with the guidelines of the Canadian Council on Animal Care and approved by the Queen's University Animal Care Committee. Each rat was used for only one experiment.

Experimental procedures were conducted under deep urethane anesthesia $(1.5 \mathrm{~g} / \mathrm{kg}$, intraperitoneal [i.p.], administered in three $0.5 \mathrm{~g} / \mathrm{kg}$ doses every $20 \mathrm{~min}$ and supplemented with $0.2-0.6 \mathrm{~g} / \mathrm{kg}$ as required prior to the onset of data collection). Rats were placed in a stereotaxic 
apparatus and body temperature was maintained between 36 and $37^{\circ} \mathrm{C}$ by means of an electrical heating blanket. An incision was made to expose the skull surface and small skull holes were drilled above the CA3 region (AP -4.16, ML -3.0, V -4.0), the contralateral CA1 area (for recordings in stratum radiatum: $\mathrm{AP}-4.16, \mathrm{ML}+3.0, \mathrm{~V}-3.0$; for stratum oriens: $\mathrm{AP}-4.30, \mathrm{ML}-$ 3.0, $\mathrm{V}-2.5$ ), and at bregma point (AP 0.0, ML 0.0, V - 6.5) to gain access to the MS. Skull holes for ground and reference electrodes (jewellery screws attached to miniature connectors) were made in the bone overlying the prefrontal cortex and cerebellum, respectively. An additional skull hole for a septal stimulation return electrode (jewellery screw) was drilled adjacent to bregma. All stereotaxic measurements were based on the anatomical work of Paxinos and Watson (1998). Final ventral placements of the CA3 stimulation and the CA1 recording electrodes were adjusted to elicit maximal amplitude field excitatory post-synaptic potentials (fEPSPs) and paired-pulse facilitation (100 ms interstimulus interval, ISI) in area CA1 in response to contralateral CA3 stimulation.

\subsubsection{Electrophysiology}

Stimulation of CA3 $(0.2 \mathrm{~ms}$ pulses every $30 \mathrm{~s}$, intensity adjusted to yield $50-60 \%$ of maximal fEPSP amplitude) was provided by a concentric bipolar electrode (Rhodes Medical Instruments Series 100, David Kopf, Tujunga, CA, USA) connected to a stimulus isolation unit providing a constant current output (PowerLab/16 s system with ML 180 Stimulus Isolator, AD Instruments, Toronto, Canada). MS stimulation was provided through a monopolar electrode (Teflon insulated stainless steel, 125 - $\mu \mathrm{m}$ tip diameter) delivering single, negative constant-current pulses (0.2 ms duration, $1.0 \mathrm{~mA})$.

Typically, fEPSPs were recorded in stratum radiatum of CA1, but some animals had a second electrode placed in stratum oriens. All fEPSPs were differentially recorded (Teflon 
insulated stainless steel wire, $125-\mu \mathrm{m}$ tip diameter) against a reference electrode placed in the bone overlaying the cerebellum. The CA1 signals were amplified, filtered $(0.3-1 \mathrm{kHz})$, digitized $(10 \mathrm{kHz})$, and stored for subsequent off-line analysis using the PowerLab system running Scope software (v. 4.0.2).

In a subset of animals, paired-pulse stimuli ( $0.2 \mathrm{~ms}$ pulse duration, $50 \mathrm{msec}$ ISI, every 30 $\mathrm{s}$, intensity adjusted to yield $50-60 \%$ of maximal fEPSP amplitude) were used throughout the experiment, rather than single test pulses, as described above.

\subsubsection{Experimental Procedures}

Prior to formal data collection, input-output curves were established for each animal by recording fEPSPs in CA1 in response to CA3 stimulation between 0.1 and $1.0 \mathrm{~mA}(0.1 \mathrm{~mA}$ increments). Based on these input-output curves, a stimulation intensity eliciting between $50-60 \%$ of the maximal fEPSP amplitude was chosen for the subsequent experiment. For each rat, 60 initial baseline fEPSPs (every $30 \mathrm{~s}$ ) were recorded. Subsequently, the MS-CA3 alternating stimulation protocol was delivered, consisting of 50 single pulses to both the MS and CA3 area, delivered at $0.5 \mathrm{~Hz}$, with the ISI between successive MS and CA3 pulses ranging from 100-1500 ms. Typically, recordings of fEPSPs in CA1 (every $30 \mathrm{~s}$, as in baseline) in response to single pulse CA3 stimulation continued for two hours following the alternating stimulation protocol. However, in a subset of animals, recordings continued for a total of four hours. Further, in an additional group of animals, the effect of repeated episodes of alternating MS-CA3 stimulation was examined to assess the saturation point of synaptic potentiation. We also studied the effects of repeated single pulse stimulation ( 50 pulses at $0.5 \mathrm{~Hz}$, as above) of either the MS or CA3 alone to examine if co-activation of these areas is required to elicit synaptic potentiation. 
For experiments assessing the role of cholinergic (muscarinic and nicotinic) and NMDA receptors in synaptic changes elicited by alternating MS-CA3 stimulation (using an ISI of 1000 $\mathrm{ms})$, different groups of rats received scopolamine ( $5 \mathrm{mg} / \mathrm{kg}$, i.p. $)$, mecamylamine $(5 \mathrm{mg} / \mathrm{kg}$, i.p) or MK-801 (0.5 mg/kg, i.p.). All drugs (obtained from Sigma/Research Biochemicals, Oakville, Ontario, Canada) were dissolved in saline and administered 30 min prior to the commencement of baseline fEPSP recordings. In cases when antagonists produced significant changes to MS-CA3 induced synaptic enhancement, additional experiments were performed to verify that drugs did not exert significant, suppressant effects on baseline (i.e., non-potentiated) fEPSPs.

Local drug application in CA1 was performed using a reverse microdialysis probe (Mab. 2.14.4; 2-mm active polyethersulfane membrane length, 35-kDa cutoff; S.P.E. Ltd, North York, ON,Canada) placed immediately adjacent to the recording electrode, with the probe tip extending about $1 \mathrm{~mm}$ below the tip of the electrode. The dialysis probe was connected to a Hamilton microsyringe driven by a microdialysis pump (CMA/102, CMA Microdialysis, Solna, Sweden) and continuously perfused with artificial cerebral spinal fluid (aCSF) $(1.0 \mu \mathrm{L} / \mathrm{min})$ composed of (in mM): $118.3 \mathrm{NaCl}, 4.7 \mathrm{KCl}, 1.2 \mathrm{MgSO} 4,1.2 \mathrm{NaH} 2 \mathrm{PO} 4,2.5 \mathrm{CaCl} 2,10.0$ glucose, 22.1

NaHCO3. For the purpose of NMDA receptor blockade, aCSF containing dl-amino-5phosphonopentanoic acid (AP-V) (10 mM, Sigma/RBI) was applied. Reverse dialysis allows local drug application by means of concentration-driven diffusion of drug molecules through the dialysis probe membrane into the surrounding neural tissue, with estimated drug concentrations outside the membrane reaching approximately $10 \%$ of aCSF concentrations (e.g. Oldford \& Castro-Alamancos, 2003). 
At the end of each experiment, rats were perfused through the heart with $10 \%$ formalin, their brains were extracted, and standard histological techniques were used to verify all electrode placements. Data obtained with inaccurate placements were excluded from the data analysis.

\subsubsection{Data Analysis}

All data are expressed as a mean \pm standard error of mean. The maximal fEPSP amplitude was analyzed offline by the Scope software. Subsequently, amplitude data were averaged over 10-min intervals, and these averages were normalized by dividing all data for each rat by the average baseline (pre-MS-CA3 alternating stimulation) amplitude of that animal. Paired pulse facilitation (PPF) ratio is expressed as the maximum amplitude of the second fEPSP divided by the amplitude of the first fEPSP in a pair. The change in PPF ratio over time was calculated as that PPF average in the last $30 \mathrm{~min}$ of the total recording period minus the initial PPF ratio during baseline (prior to alternating MS-CA3 stimulation). For statistical analyses, analyses of variance (ANOVA) and, where statistically appropriate, simple effects tests were computed using the software package CLR ANOVA (v.1.1, Clear Lake Research Inc., Houston, TX). A linear regression test was used for correlational analyses.

\subsection{Results}

\subsubsection{Alternating single pulse stimulation of the MS and CA3 elicits an increase in fEPSP amplitude in CA1 (MS-H LTP)}

Single pulses applied to area CA3 produced positive-going fEPSPs (latency to peak about 10-11 ms) recorded in the contralateral stratum radiatum of the CA1 area, while fEPSPs recorded in stratum oriens consisted of phase-reversed, negative-going potentials (see Fig. 1). These fEPSP 
characteristics are equivalent to those previously reported as apical and basilar dendritic fEPSPs on CA1 pyramidal cells (Leung et al., 2003; Ovsepian et al., 2004). The dipole field in these recordings is typically attributed to excitation at the basal dendrites after stimulation of the CA3 afferents (Leung et al., 2003). The data reported below refer to recordings taken in stratum radiatum, except where noted otherwise.

In rats receiving only $\mathrm{CA} 3$ stimulation throughout the experiment $(n=8$; single test pulses every $30 \mathrm{~s}$ plus 50 single pulses at $0.5 \mathrm{~Hz}$ during the induction phase), fEPSP amplitude in stratum radiatum remained relatively stable throughout the entire recording period (Fig.1; average fEPSP amplitude of $105 \pm 4.0 \%$ of baseline during the last $30 \mathrm{~min}$ of the experiment). However, alternating single pulses applied to the MS and CA3 $(n=14$; a total of 50 pulses at $0.5 \mathrm{~Hz}$ for each, starting with MS and followed by CA3 stimulation, $1000 \mathrm{~ms}$ ISI) produced a gradual, significant increase of fEPSP amplitude over the $2 \mathrm{~h}$ period following MS-CA3 stimulation (Fig. 1; average fEPSP amplitude of $123 \pm 4.5 \%$ of baseline during the final $30 \mathrm{~min}$ ). In a subset of these experiments $(n=4)$, the stability of synaptic enhancement was assessed by continuing fEPSP recordings over a $4 \mathrm{~h}$ period after induction. In these experiments, enhancement was maintained for the entire recording period (average of 120\% of baseline between 3.5 and $4 \mathrm{~h}$ following induction; data not shown). In an additional control group, stimulation of the MS (50 pulses at $0.5 \mathrm{~Hz} ; n=6$ ) in the absence of CA3 pulses during induction did not elicit significant changes in fEPSP amplitude (104\% of baseline during the last $30 \mathrm{~min}$; simple effect of time, $F_{14}$, ${ }_{70}=1.202, P=0.2942 ;$ data not shown $)$.

A potentiation effect very similar to that in stratum radiatum was also observed for fEPSPs recorded in stratum oriens $(n=7)$, where MS-CA3 stimulation again elicited a gradually developing increase in fEPSP amplitude to a maximum of $123 \%$ of baseline during the last 30 
min of the experiment (Fig. 1). Thus, these experiments demonstrate that MS-H LTP constitutes a long-lasting form of synaptic potentiation that requires co-activation of medial septal and CA3

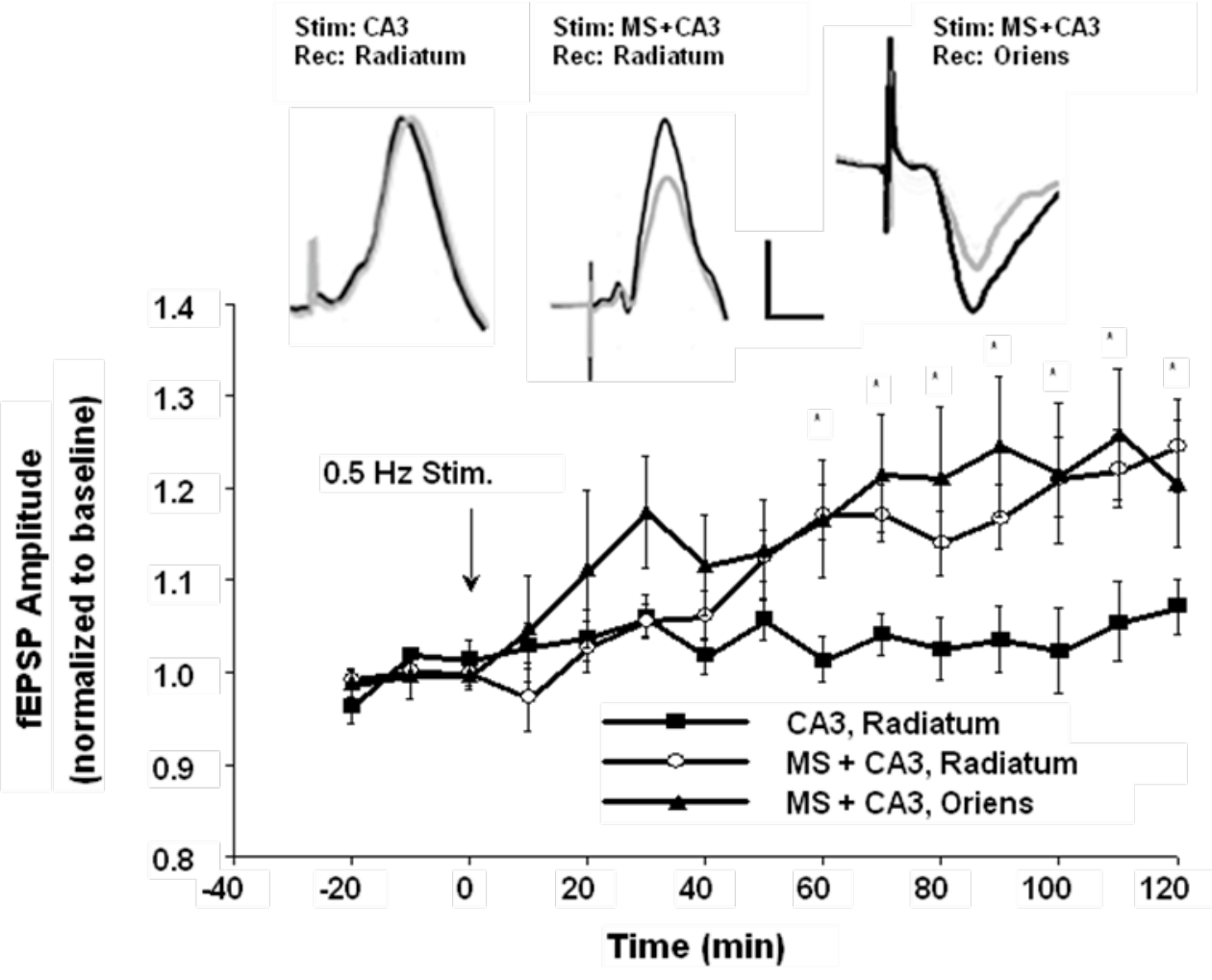


Figure 1. Effects of alternating medial septum (MS)-CA3 stimulation on amplitude of field excitatory postsynaptic potentials (fEPSPs) recorded in CA1 of urethane-anaesthetized rats. Alternating single pulse stimulation (Alternating Stim., at arrow) of the MS and CA3 area (a total of 50 pulses at $0.5 \mathrm{~Hz}$ for both, starting with MS and followed by CA3 stimulation, $1000 \mathrm{~ms}$ inter-stimulus interval) resulted in a gradual increase in fEPSP amplitude in both strata radiatum $(\mathrm{n}=14)$ and oriens $(\mathrm{n}=7)$ over the $2 \mathrm{~h}$ period following alternating stimulation. Single pulses applied to CA3 in the absence of MS stimulation failed to induce synaptic enhancement in stratum radiatum ( 50 single pulses to $\mathrm{CA} 3,0.5 \mathrm{~Hz} ; \mathrm{n}=8)$. Inserts depict typical fEPSPs during baseline (grey) and at the end of the experiment (black) in animals receiving MS and CA3 stimulation (Stim.) for recordings (Rec.) in stratum radiatum (middle), stratum oriens (right), as well as control animals receiving only CA3 stimulation (left, recordings in stratum radiatum). Note the enhanced fEPSP amplitude in rats receiving the alternating protocol (fEPSPs are averages of 10 individual sweeps, calibration is $10 \mathrm{~ms}$ and $1 \mathrm{mV}$ ). Statistics: MS + CA3, radiatum group vs. CA3, radiatum group: effect of group, $F_{1,20}=6.2, P=0.02$; effect of time, $F_{14,280}=8.3$, $P<0.0001$; group by time interaction, $F_{14,280}=4.9, P<0.0001$; simple effect of time significant for animals receiving MS-CA3 stimulation $\left(F_{14,350}=15.0, P<0.0001\right)$ but not for animals receiving CA3 stimulation alone $\left(F_{14,98}=1.2, P=0.276\right) .{ }^{*}$ denote significant $(P<0.05)$ group differences. Stratum oriens group also showed a significant effect of time, $F_{14,84}=4.28, \mathrm{P}<$ 0.0001, and the level of potentiation did not differ from that seen in the stratum radiatum group, $F$ $1,19=0.69, P=0.415$ 
fibers to elicit enhancement of synaptic responses of CA1 neurons. Next, we examined the effects of changing the ISI between alternating MS-CA3 pulses (delivered at $0.5 \mathrm{~Hz}$, as above, all ISIs refer to interval between the initial MS pulse and the subsequent CA3 pulse) during induction. Surprisingly, ISIs of $500(n=4), 750(n=5)$, and $1500 \mathrm{~ms}(n=6)$ did not result in significant changes of fEPSP amplitude relative to baseline, while an ISI of $100 \mathrm{~ms}(n=5)$ produced significant synaptic depression (Fig. 2). Thus, relatively strict temporal requirements apply to the induction of MS-H LTP, with ISIs of about $1000 \mathrm{~ms}$ apparently required in order to elicit this form of synaptic potentiation.

In an attempt to examine whether repeated episodes of alternating MS-CA3 stimulation can elicit greater levels of potentiation than those seen with a single induction episode, we assessed the effect of multiple MS-CA3 induction episodes, delivered at $2 \mathrm{~h}$ intervals (Fig. 3; two induction episodes over $4 \mathrm{~h}, n=15$, three induction episodes over $6 \mathrm{~h}, n=5)$. Amplitude of fEPSPs averaged over 1.5 to $2 \mathrm{~h}$ after each of the first, second, and third induction episodes were $15 \%, 31 \%$, and $31 \%$, respectively (Fig. 3; insert). Consequently, two induction episodes resulted in the maximal enhancement of synaptic responses of CA1 neurons. 


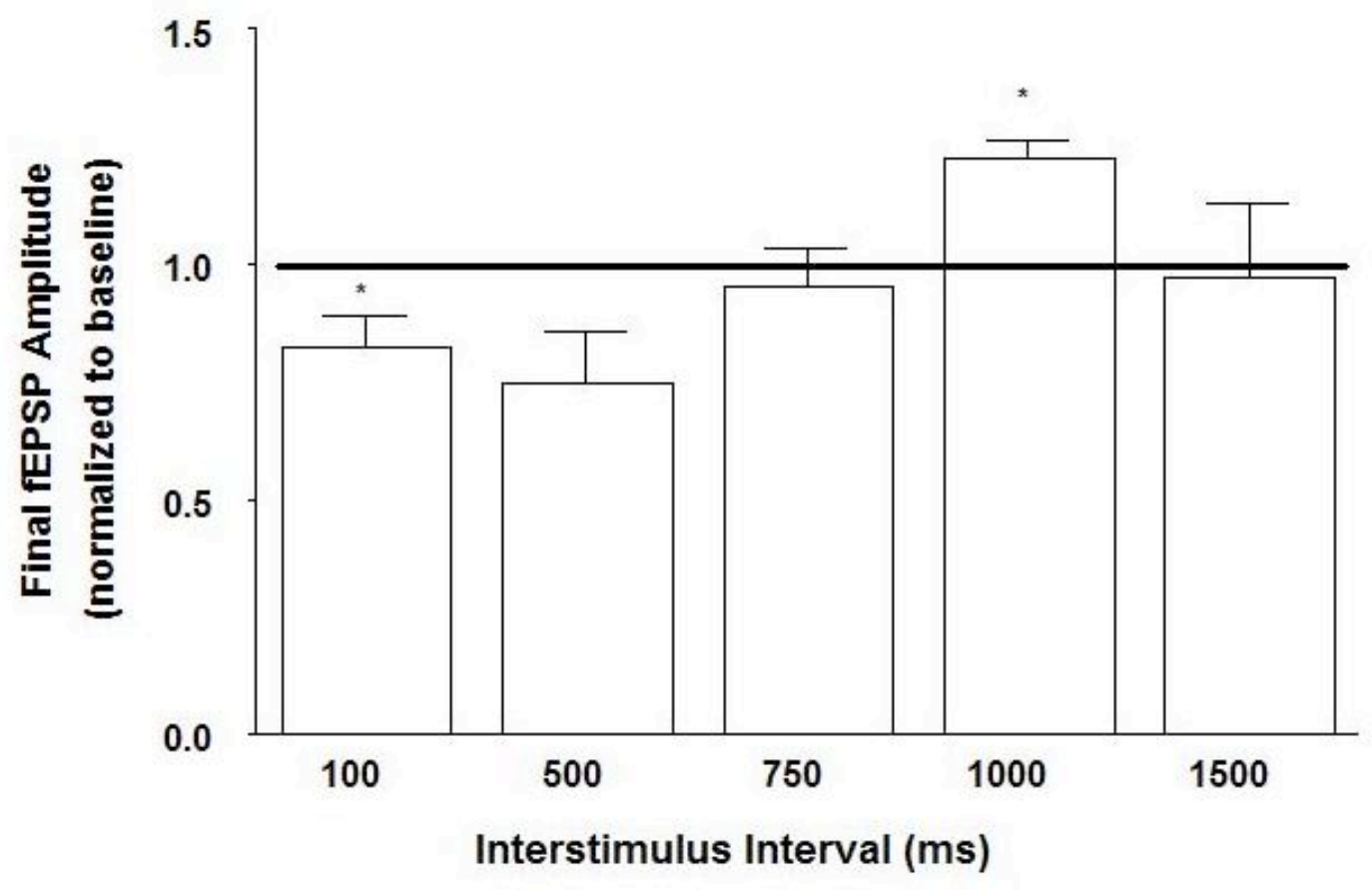


Figure 2. The effects of various interstimulus intervals (ISIs) between alternating stimulation of the medial septum (MS) and CA3 area on amplitude of CA1 field excitatory postsynaptic potentials (fEPSPs) averaged between 1.5 and $2 \mathrm{~h}$ following the alternating stimulation.

ISIs of $100 \mathrm{~ms}(n=5)$ and $1000 \mathrm{~ms}(n=14)$ resulted in significant synaptic depression and enhancement, respectively. All other ISIs (500 ms, $n=4 ; 750 \mathrm{~ms}, n=5 ; 1500 \mathrm{~ms}, n=6)$ did not induce significant changes in fEPSP amplitude. Statistics: effect of time comparing fEPSP amplitude values averaged over the $30 \mathrm{~min}$ baseline period and the last $30 \mathrm{~min}$ of recording (i.e., 1.5 to $2 \mathrm{~h}$ following induction), $100 \mathrm{~ms}, F_{1,4}=19.6, P=0.01 ; 500 \mathrm{~ms}, F_{1,3}=5.4, P=0.102 ; 750$ ms, $F_{1,4}=0.4, P=0.592 ; 1000 \mathrm{~ms}, F_{1,13}=26.8, P=0.0002 ; 1500 \mathrm{~ms} F_{1,5}=0.04, P=0.856 . *$ denotes significant changes from baseline fEPSP amplitude. 


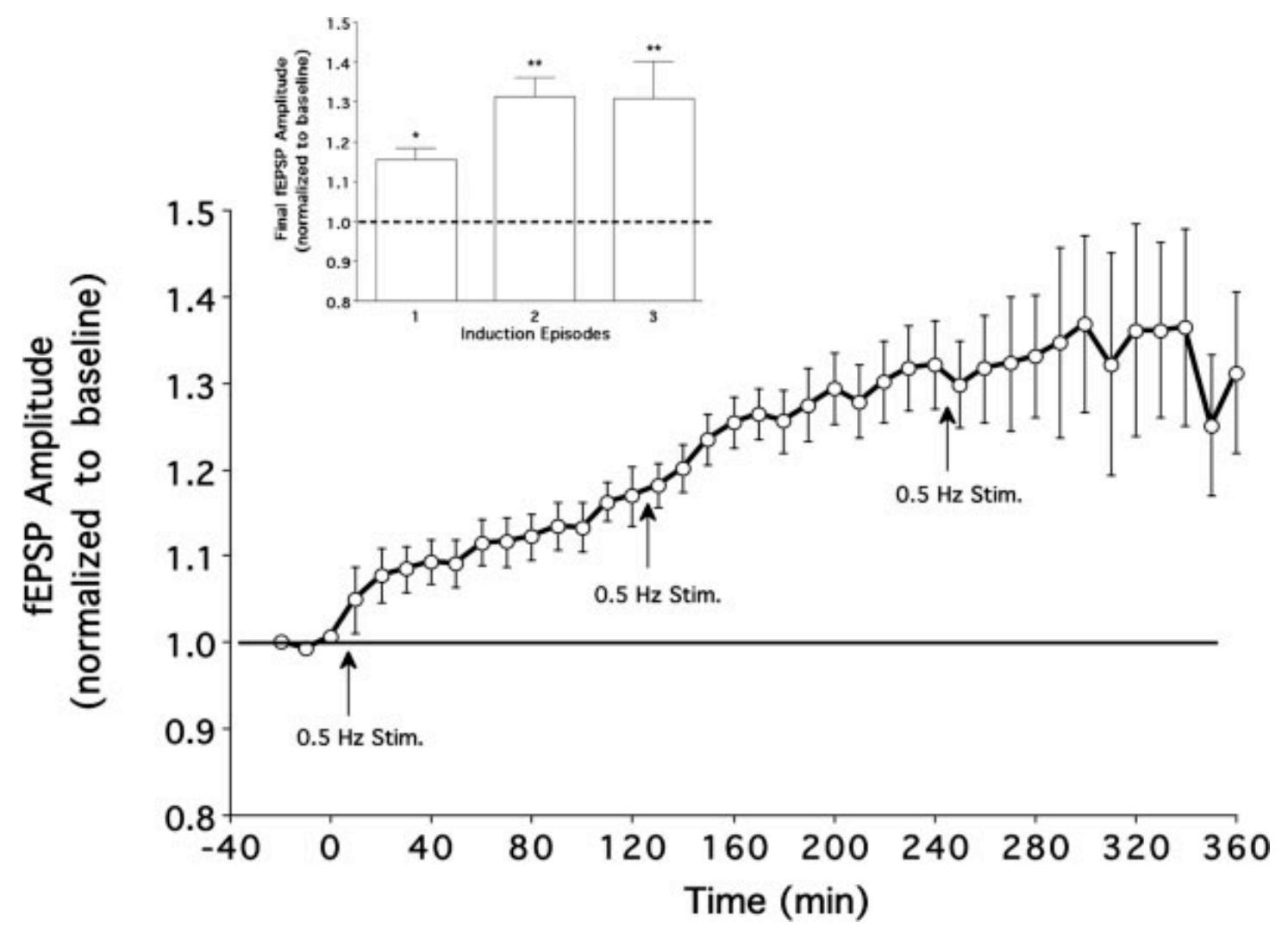

Figure 3. The effects of multiple induction episodes of alternating stimulation of the medial septum (MS) and CA3 area on amplitude of CA1 field excitatory postsynaptic potentials (fEPSPs).

Repetition of the alternating MS-CA3 stimulation protocol (at arrows) every $2 \mathrm{~h}$ (50 MS-CA3 single pulse stimulations, $1000 \mathrm{~ms}$ interstimulus interval, $0.5 \mathrm{~Hz}$ per induction) enhanced fEPSP amplitude to $115 \%, 131 \%$, and $131 \%$ of baseline during the last half hour following one, two, and three induction episodes, respectively. Statistics: effect of time for $4 \mathrm{~h}, F_{26,234}=13.5, P<0.001, n$ $\left.=15 ; 6 \mathrm{~h}, F_{38,152}=8.4, P<0.001, n=5\right)$; Insert shows fEPSP amplitude averaged over 1.5 to $2 \mathrm{~h}$ following each of the three induction episodes; * and ** indicates significant differences $(P<$ 0.05 ) from baseline and baseline plus one induction episode, respectively. 


\subsubsection{MS-H LTP is dependent on NMDA, but not cholinergic receptor activation}

Systemic administration of the NMDA receptor antagonist MK-801 (0.5 mg/kg, i.p., given 30 min prior to the onset of baseline recordings; $n=6$ ) completely prevented synaptic potentiation elicited by MS-CA3 alternating stimulation (Fig. 4). In fact, it appeared as if the alternating stimulation protocol resulted in synaptic depression after MK-801 treatment, even though this effect did not reach statistical significance (see figure caption for statistics). Control experiments showed that, in rats receiving only CA3 stimulation without additional MS stimuli ( $n$ $=4)$, MK-801 administration did not significantly suppress fEPSP amplitude over time $(F$ 's $<0.8$, $P>0.7$ for effects of time, group, and interaction comparing rats receiving only CA3 stimulation without drug treatment vs. MK-801, data not shown). Thus, nonspecific depressant effects on CA3-CA1 transmission during the course of the experiment do not account for the inhibition of synaptic enhancement following MK-801 treatment.

Further experiments were conducted to assess the effects of systemic cholinergic receptor blockade (Fig. 5). Surprisingly, enhancement of fEPSP amplitude elicited by MS-CA3 alternating stimulation was not significantly affected by administration of mecamylamine $(5 \mathrm{mg} / \mathrm{kg}$, i.p., administered $30 \mathrm{~min}$ prior to onset of baseline recordings, $\mathrm{n}=9)$ or scopolamine $(5 \mathrm{mg} / \mathrm{kg}$, same administration regime, $\mathrm{n}=7$ ). Thus, it appears that muscarinic or nicotinic receptor stimulation is not required for the enhancement of synaptic strength seen in these experiments. 


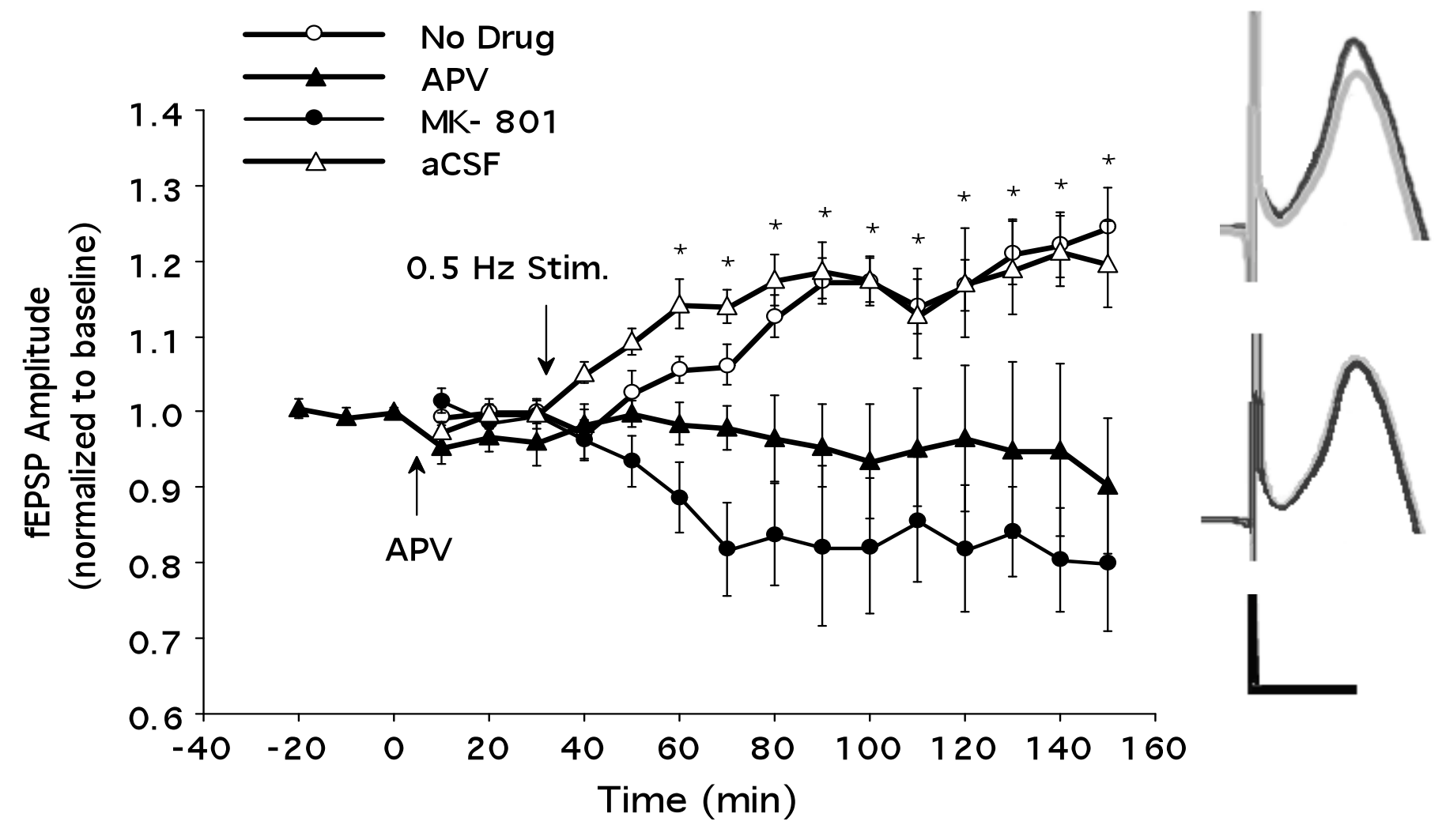


Figure 4. Effect of MK-801 and AP-V treatment on changes in field excitatory postsynaptic potential (fEPSP) amplitude following alternating medial septum (MS)-CA3 stimulation. Systemic administration of MK-801 $(0.5 \mathrm{mg} / \mathrm{kg}$, i.p., $n=6) 30 \mathrm{~min}$ prior to onset of baseline recordings prevented synaptic enhancement during the $2 \mathrm{~h}$ period following alternating MS-CA3 stimulation (at arrow; 50 single MS-CA3 pulses at $0.5 \mathrm{~Hz}, 1000 \mathrm{~ms}$ interstimulus interval). No drug group $(n=14)$ is same as in Fig. 1. In the presence of artificial cerebrospinal fluid (aCSF, $n$ $=5$, applied in CA1 by reverse microdialysis), alternating MS-CA3 stimulation resulted in a gradual increase in fEPSP amplitude that was not significantly different from the no drug group. In contrast, local application of AP-V in CA1 $(10 \mathrm{mM}, n=5)$ completely blocked the increase in fEPSP amplitude elicited by MS-CA3 stimulation. Inserts depict fEPSPs during baseline (grey) and at the end of the experiment (black) for an animal receiving CA1 application of aCSF (top) or AP-V (bottom). Note the blockade of synaptic potentiation in the rat receiving AP-V (fEPSPs are averages of 10 individual sweeps, calibration is $10 \mathrm{~ms}$ and $0.5 \mathrm{mV}$ ). Statistics: MK-801 vs. No Drug, effect of group, $F_{1,18}=16.7, P=0.0007$; effect of time, $F_{14,252}=1.5, P=0.331$; group by time interaction, $F_{14,252}=7.6, P<0.0001$; simple effect of time for MK-801 treated rats, $F_{14,252}=$ $1.3, P=0.182$. AP-V vs. aCSF, effect of group, $F_{1,8}=8.9, P=0.017$; effect of time, $F_{14,112}=$ $2.4, P=0.860$; group by time interaction, $F_{14,112}=2.4, P=0.005$; simple effect of time for aCSF, $F_{14,56}=6.8, P<0.0001 ; \mathrm{AP}-\mathrm{V}, F_{14,56}=0.2, P=0.998, *$ indicates significant $(P<0.05)$ difference between aCSF and AP-V groups. 


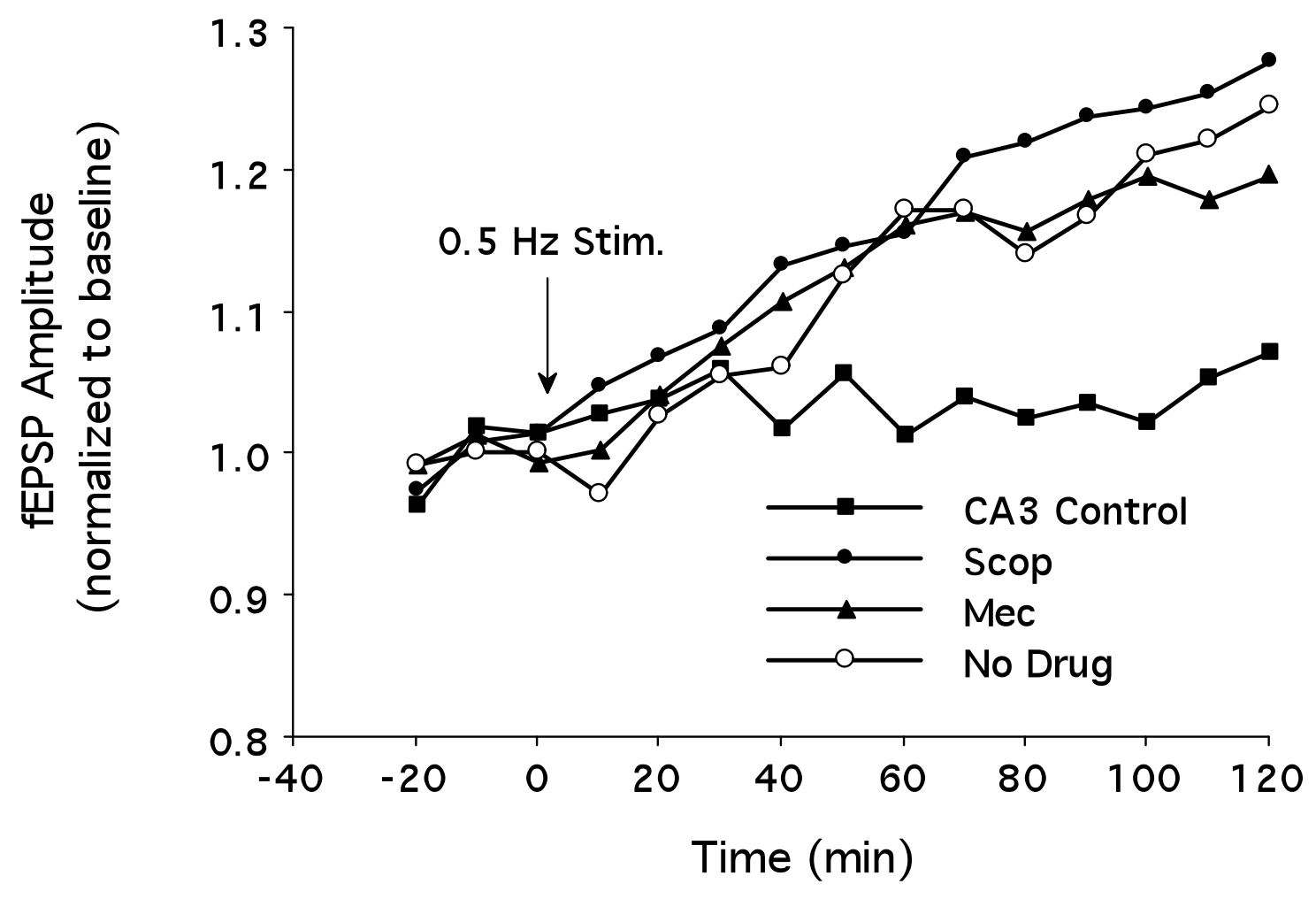


Figure 5. The effects of scopolamine and mecamylamine on enhancement in field excitatory postsynaptic potential (fEPSP) amplitude in CA1 following medial septum (MS)-CA3 alternating stimulation.

Enhancement of fEPSP amplitude elicited by MS-CA3 stimulation (at arrow, same parameters as in Fig. 1) was resistant to administration of scopolamine (Scop., $5 \mathrm{mg} / \mathrm{kg}$, i.p., $\mathrm{n}=7$ ) or mecamylamine (Mec., $5 \mathrm{mg} / \mathrm{kg}$, i.p., $\mathrm{n}=9$ ). Both drugs were administered $30 \mathrm{~min}$ before the onset of baseline recordings. Statistics: ANOVA comparing mecamylamine and untreated rats, effect of group, $F_{1,21}=0.003, P=0.962$, effect of time, $F_{14,294}=20.2, P<0.001$, group by time interaction, $F_{14,294}=0.4, P=0.962 ;$ scopolamine vs. untreated rats, effect of group, $F_{1,19}=1.0, P$ $=0.34$, effect of time, $F_{14,266}=20.1, \mathrm{P}<0.001$, group by time interaction, $F_{14,266}=0.5, P=$ 0.922; simple effects of time significant for untreated rats and rats receiving scopolamine or mecamylamine, $F>8.0, P<0.001$; No Drug group and CA3 control group are same as in Fig.1; error bars omitted for clarity. 


\subsubsection{Enhancement of fEPSP amplitude is associated with decreases in PPF ratio.}

In order to assess the relative contributions of pre- and postsynaptic changes in fEPSP enhancement following MS-CA3 alternating stimulation, we examined changes in PPF ratio (two test pulses throughout the experiment, $50 \mathrm{~ms} \mathrm{ISI,} n=10$ ) in a subset of animals that received two MS-CA3 induction episodes $2 \mathrm{~h}$ apart. In these experiments, fEPSP amplitude increased to $115 \%$ and $127 \%$ of baseline for the first and second induction episodes, respectively, while PPF ratio was $104 \%$ and $93 \%$ of the baseline ratio at these time points (Fig. 6A). The raw PPF ratios were 1.89 during baseline, 1.97 following one induction episode, and 1.76 following the second induction episode, calculated as amplitude of the second fEPSP/amplitude of the first fEPSP in a pair (all values are averages over the last $30 \mathrm{~min}$ of the $2 \mathrm{~h}$ period following each of the two induction episodes). Correlation analyses of these data revealed that there was no significant relation between fEPSP amplitude and PPF measures after one induction episode (Fig. 6B). However, there was a significant, inverse correlation between the fEPSP enhancement and PPF ratio after the second induction episode, i.e., the final $30 \mathrm{~min}$ of the experiment (Fig. 6C). At this time point, changes in PPF ratio accounted for $46 \%$ of the total enhancement of fEPSP amplitude induced by alternating MS-CA3 stimulation (see figure caption for statistics). 


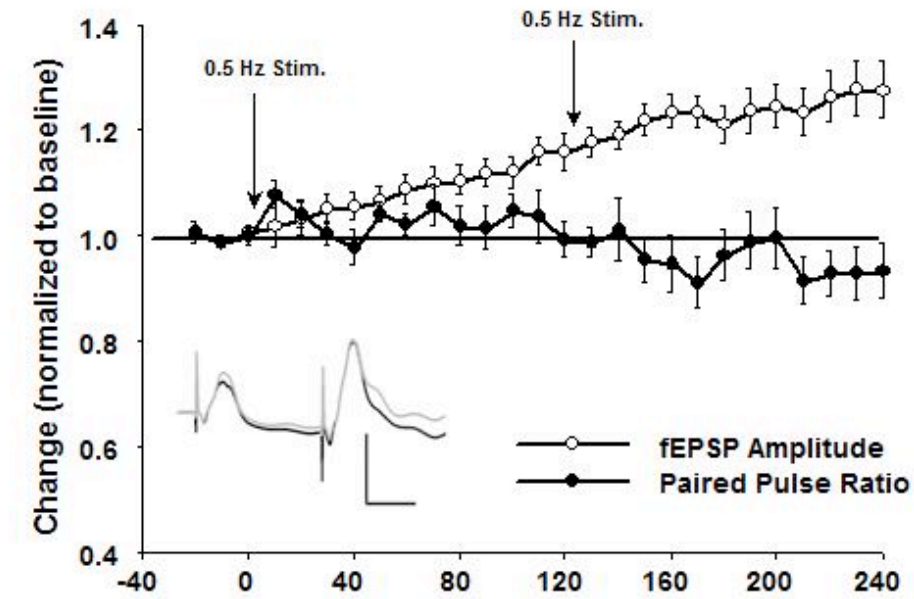

(A)

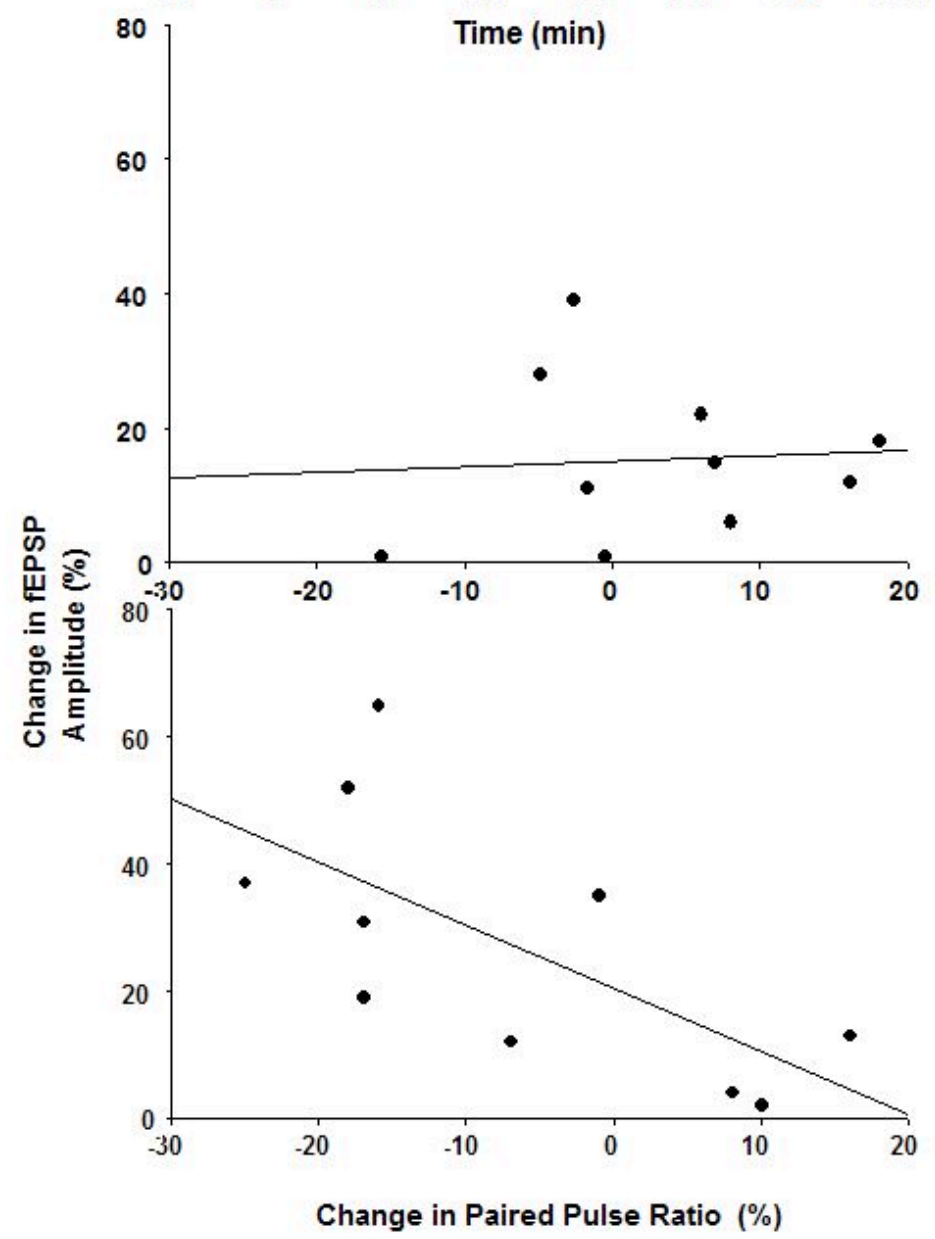

(B) 
Figure 6. Changes in paired pulse facilitation (PPF) ratio and field excitatory postsynaptic potential (fEPSP) amplitude in CA1 following medial septum (MS)-CA3 alternating stimulation.

(A) Two episodes of MS-CA3 stimulation (at arrows, same parameters as in Fig. 1) resulted in a significant enhancement of fEPSP amplitude, and a significant decrease in the PPF ratio $(n=10$, paired pulses delivered throughout the experiment, $50 \mathrm{~ms}$ interstimulus interval, data are a subset of animals shown in Fig. 3). Inserts depict fEPSPs during baseline (black) and at the end of the experiment (grey). Note the increase in fEPSP amplitude in response to the first stimulation pulse, accounting for the decrease in PPF ratio (fEPSPs are averages of 10 individual sweeps, calibration is $20 \mathrm{~ms}$ and $1 \mathrm{mV}$ ). (B) Correlation between change in fEPSP amplitude and PPF ratio after one induction episode (values averaged between 1.5-2 h following the first induction; PPF ratio change $=$ final PPF ratio minus initial PPF ratio (averaged during baseline)/initial PPF ratio *100). (C) Correlation between changes in fEPSP amplitude and PPF ratio $1.5-2 \mathrm{~h}$ after the second induction episode (data calculated as in panel B). Statistics: (A) fEPSP amplitude, effect of time, $F_{26,234}=$ 13.5, $P<0.001$; PPF ratio, effect of time, $F_{26,234}=2.2, P=0.001$; (B) The best-fit linear regression line is plotted, $\mathrm{y}=15.065+0.079776 \mathrm{x}, r=0.07, r^{2}=0.005 ;$ (C) $\mathrm{y}=20.312-0.99828 \mathrm{x}, r=-0.68$, $r^{2}=0.46, P=0.03$ 


\subsection{Discussion}

The majority of studies examining plasticity in the hippocampal formation have used LTP protocols that require high frequency stimulation of glutamatergic afferents to induce longlasting changes in synaptic efficacy. Here, we characterize a novel form of long-lasting synaptic plasticity, present in both strata radiatum and oriens of CA1, elicited by alternating, LFS of the MS and CA3 area (MS-H LTP). MS-H LTP has a slow onset, can be saturated with repeated induction episodes, and appears to be optimal at unusually long (1000 ms) ISIs between successive MS and CA3 stimuli. Pharmacological experiments demonstrate a critical role of hippocampal NMDA receptor activation, but apparently no critical involvement of cholinergic mechanisms with the induction protocol used here. Interestingly, data from the paired-pulse experiments suggest that MS-H LTP is comprised of two processes, with the initial synaptic enhancement occurring in the absence of changes in PPF ratio, while later enhancement is related to a decrease in PPF ratio. Consequently, initial postsynaptic changes might be followed by changes in transmitter release from terminals of presynaptic CA3 neurons. To the best of our knowledge, these experiments are the first to describe long-lasting enhancement at CA3-CA1 hippocampal synapses using low frequency, alternating stimulation of two separate afferent systems in an intact in vivo preparation.

Typically, enhancement of hippocampal synapses is induced by various forms of high frequency or theta-burst stimulation protocols, while LFS (e.g., $1 \mathrm{~Hz}$ ) generally results in synaptic depression (Malenka and Bear, 2004; Massey and Bashir, 2007; see also Thiels et al., 1994). However, recent evidence has shown that some synapses exhibit long-lasting potentiation in response to LFS protocols. Lanté et al. (2006a,b) showed that, in hippocampal slices, $1 \mathrm{~Hz}$ stimulation of the CA1 area applied for 5 min produced a gradual, slow-onset synaptic 
potentiation of local CA1 synapses in close proximity to the stimulation site. This enhancement of fEPSPs occurred in area CA1, but not CA3 or dentate gyrus, and was independent of NMDA receptor activation (Lanté et al. 2006a, b). Similarly, Huang and Kandel (2007) described a slowdeveloping, NMDA-independent form of synaptic potentiation following $1 \mathrm{~Hz}$ stimulation of cortico-lateral amygdala fibers in vitro, a form of LTP that requires the co-activation of glutamatergic and various neuromodulatory inputs including serotonin, dopamine and noradrenalin. Together, these findings suggest that long-lasting synaptic potentiation can be elicited by low frequency stimulation, results that are intriguing since low frequency activity mimics some aspects of spontaneous oscillatory patterns present in hippocampal neurons (Vanderwolf, 1969; Buzsáki et al., 1983; Buzsáki, 1989).

Interestingly, while previous work suggests that hippocampal and amygdaloid LTP elicited by LFS stimulation is not dependent on NMDA receptors activation (Lanté et al. 2006a,b; Huang and Kandel, 2007), our findings indicate that MS-H LTP does not occur in the presence of the NMDA receptor antagonist MK-801 (systemic) or AP-V (locally applied in CA1). Consequently, it appears that multiple receptor and signaling mechanisms can trigger LTP-like synaptic enhancement in response to low frequency inputs. Based on our data, we speculate that the repeated co-activation of septal and commissural inputs constitutes an effective mechanism of NMDA receptor activation in CA1. Electrophysiological and anatomical studies have provided evidence for glutamatergic projections from the MS to the hippocampal formation (Sotty et al., 2003; Colom et al., 2005), which may play a role in cellular synchrony and generation of the hippocampal theta rhythm (Bland et al., 2007) independent of cholinergic and GABAergic projections. Whether this glutamatergic projection system plays a role in the induction of MS-H LTP is unknown, even though this hypothesis is consistent with the lack of effectiveness of high 
doses of cholinergic (muscarinic and nicotinic) receptors antagonists to suppress this form of synaptic potentiation. Clearly, for the induction protocol used here, engagement of cholinergic fibers between MS and CA1 is not a critical prerequisite for MS-H LTP to occur, even though it is possible that changes to the protocol might reveal potential cholinergic contributions to MS-H LTP. Work by Straube and Frey (2003) has shown that the relative contributions of NMDA and other, neuromodulatory signals to the induction of heterosynaptic LTP depend on strength of the induction protocol, with weaker protocols showing greater dependence on neuromodulatory LTP reinforcement (see also Huang and Kandel, 2007). Whether changes to the stimulation parameters used for the alternating MS-CA3 protocol reveal an involvement of cholinergic mechanisms remains to be determined.

To us, the most surprising finding of these experiments was the fact that unusually long ISIs (1000 ms) between consecutive septal and CA3 stimuli were required to elicit MS-H LTP. This time window is well beyond those normally assumed to be effective in inducing NMDA receptor-dependent types of synaptic strengthening such as LTP and spike-time dependent plasticity (Larson and Lynch, 1988; Bennett, 2000; Dan and Poo, 2004; Malenka and Bear, 2004; Bi and Rubin, 2005; Yao and Dan, 2005; see also Massey and Bashir, 2007). However, there are some types of synaptic facilitation that exhibit fairly broad temporal windows for the integration of separate synaptic inputs to trigger NMDA-dependent plasticity. Popescu et al. (2007) characterized a form of heterosynaptic facilitation induced by pairing of corticostriatal and amygdala inputs onto single, striatal medium spiny neurons in vitro. Long-lasting ( $>30 \mathrm{~min}$ ) potentiation following repeated pairing of these convergent inputs was NMDA-dependent and also showed a surprisingly broad time window between cortical and amygdaloid stimuli of up to $500 \mathrm{~ms}$. Thus, there is growing evidence that some forms of NMDA-dependent plasticity exhibit 
highly unconventional temporal dynamics with regard to the integration of synaptic inputs that are effective in eliciting long-lasting potentiation. The fact that we observed significant synaptic depression at short (100 ms) ISIs between MS and CA3 pulses is consistent with other reports showing that low frequency paired stimulation protocols are an effective means to induce LTD at commissural-CA1 synapse (Thiels et al., 1994).

To date, the relative contributions of pre- and postsynaptic mechanisms to different forms of hippocampal LTP continue to remain controversial (Bekkers and Stevens, 1990; Malinow and Tsien, 1990; Nicoll and Malenka, 1999; Maruki et al., 2001; Bredt and Nicoll, 2003; Malenka and Bear, 2004). We analyzed changes in the ratio of PPF, a form of short-term plasticity primarily associated with successively increasing calcium levels in the presynaptic terminal, resulting in an increased probability of transmitter release (Katz and Miledi, 1968; Foster and McNaughton, 1991; Schulz et al., 1994, 1995). A number of in vitro studies have demonstrated a relation between LTP magnitude and changes in PPF ratio, indicative of presynaptic contributions to LTP (Voronin and Kuhnt, 1990; Schulz et al., 1995, Schulz, 1997; Schulz and Fitzgibbons, 1997). Similarly, the data from our paired-pulse analysis indicated that, during the later phases of the experiment, there was an inverse correlation between the change in PPF ratio and the magnitude of synaptic enhancement. This correlation developed gradually during the course of the experiment since it was not present during the first two hours following the initial induction episode. These data indicate that changes in presynaptic functioning contribute to MS-H LTP. Given that rats used for the PPF experiments received two separate induction episodes, it remains to be determined whether the change in PPF ratios is the result of the repeated alternating stimulation given to these animals, or whether one induction episode is sufficient to engage presynaptic mechanisms. It is of interest to note, however, that the distinct temporal dynamics of 
changes in PPF ratios noted here fit well with recent evidence regarding the role of pre- and postsynaptic plasticity loci in LTP expression. Bayazitov et al. (2007) showed that, for LTP at CA3-CA1 synapses in vitro, presynaptic changes during the early phase are minimal, but become more prominent during the later phase of LTP ( $>1 \mathrm{~h}$ post induction). These experiments also indicate that NMDA receptor activation is crucial for the postsynaptic component, while voltage gated calcium channels are necessary for the expression of the delayed, presynaptic component of 'compound LTP'. Finally, Bayazitov et al. (2007) showed that the cAMP-protein kinase A cascade is necessary for the presynaptic changes associated with compound LTP, either by acting directly in presynaptic terminals to enhance transmitter release, or by influencing the production of a retrograde messenger in the postsynaptic cells. Interestingly, the cAMP-protein kinase A cascade also appears to be critical for plasticity induced by low-frequency stimulation in the hippocampus (Lanté et al., 2006a) and amygdala (Huang and Kandel, 2007). Whether any of these mechanisms are involved in MS-H LTP remains to be determined.

In summary, the present experiments describe a novel form of synaptic enhancement elicited by alternating, low frequency, single pulse stimulation of CA3 and medial septal fibers. This form of LTP is NMDA-receptor dependent, apparently does not require activation of cholinergic receptors, and may involve temporally distinct, pre- and postsynaptic mechanisms following induction. These data reinforce recent findings that long-lasting synaptic enhancement can occur in response to low frequency activity patterns, which might mimic some aspects of natural neuronal oscillations present during phases of acquisition or consolidation of memory processes (e.g., Buzsáki, 1989). 


\section{Chapter 3. Low and high frequency induced synaptic potentiation: A comparative review of induction protocols, mechanisms and implications.}

As published in "Low-frequency induced synaptic potentiation: A paradigm shift in the field of memory-related plasticity mechanisms" by Habib, D \& Dringenberg, HC. 2010. Hippocampus, Vol. 20, 29-35.

\subsection{Abstract}

Long-term potentiation (LTP) and long-term depression (LTD) are two forms of synaptic plasticity thought to play functional roles in learning and memory processes. It is generally assumed that the direction of synaptic modifications (i.e., up- or down-regulation of synaptic strength) depends on the specific pattern of afferent inputs, with high frequency activity or stimulation effectively inducing LTP, while low-frequency patterns often elicit LTD. This dogma ("high frequency-LTP, low frequency-LTD") has recently been challenged by evidence demonstrating low frequency stimulation (LFS)-induced synaptic potentiation in the rodent hippocampus and amygdala. Extensive work in the past decades has focused on deciphering the mechanisms by which high frequency stimulation of afferent fiber systems results in LTP. With this review, we will compare and contrast the well-known synaptic and cellular mechanisms underlying classical, high-frequency-induced LTP to those mediating the more recently discovered phenomena of LFS-induced synaptic enhancement. In addition, we argue that LFS 
protocols provide a means to more accurately mimic some endogenous, oscillatory activity patterns present in hippocampal and extra-hippocampal (especially neocortical) circuits during periods of memory consolidation. Consequently, LFS-induced synaptic potentiation offers a novel and important avenue to investigate cellular and systems-level mechanisms mediating the encoding, consolidation, and transfer of information throughout multiple forebrain networks implicated in learning and memory processes. 


\section{2 "Hebbian learning" with high frequency stimulation}

In 1949, the Canadian psychologist D.O. Hebb formalized his visionary postulate proposing that correlated activity in presynaptic and postsynaptic neurons is responsible for the induction of synaptic changes that take place during information storage in neuronal circuits (Hebb, 1949). Long-term potentiation (LTP) exemplifies this type of 'Hebbian Learning', as it requires coincident pre- and postsynaptic activity to result in long-lasting increases in synaptic efficacy. Experimentally, this coincident activity can be effectively mimicked by applying highfrequency (tetanic) stimulation (HFS) to glutamatergic synapses, resulting in the arrival of presynaptic signals at a partially depolarized postsynaptic membrane. Consequently, HFS (typically $100 \mathrm{~Hz}$ ) has been established as a standard protocol to induce long-lasting increases in synaptic efficacy at hippocampal and extra-hippocampal synapses (Bliss and Lómo, 1973;

Raymond, 2007; Caporale and Dan, 2008). Other commonly used induction protocols such as theta-burst stimulation (TBS) are also comprised of high frequency components that are repeated at a theta rhythm of about $5 \mathrm{~Hz}$ (Larson et al., 1986; Stäubli and Lynch, 1987). Even though the synaptic activity induced by these stimulation protocols is unlikely to completely mimic endogenous activity patterns, they have been instrumental in deciphering mechanisms underlying the induction, consolidation, and maintenance of classical LTP.

\subsection{High frequency induced LTP mechanisms: The general consensus}

The cellular and molecular mechanisms of LTP have been elucidated in great detail, in particular for area CA1 of the hippocampus. LTP in CA1 pyramidal cells induced by HFS of Schaffer collaterals/commissural fibers requires an initial activation of alpha-amino-3-hydroxy-5methyl-4-isoxazolepropionic acid (AMPA), metabotropic, and N-methyl-D-aspartate receptors (NMDAR; Bliss and Collingridge, 1993; Malenka and Nicoll, 1999; Riedel et al., 2003), which 
are often co-localized in individual, postsynaptic dendritic spines (He et al., 1998; Lissin et al., 1998). Depolarization of the postsynaptic membrane in response to AMPA receptor activation results in the removal of the voltage-dependent magnesium block of NMDA channels, allowing calcium $\left(\mathrm{Ca}^{2+}\right)$ entry into dendritic spines. The resulting rise in intracellular $\mathrm{Ca} 2+$ levels triggers the local activity of $\mathrm{Ca}^{2+}$-calmodulin-dependent protein kinase II (CaMKII) and activation of protein kinase C (PKC; Lynch et al., 1983; Malenka et al., 1988; Fukunaga et al., 1995; Izquierdo and Medina, 1995; Nicoll and Malenka, 1999; Lisman et al., 2002; for discussions of other kinases including protein kinase A (PKA) and extracellular regulated signal kinase, see Impey et al., 1998; Selcher et al., 2003; Gelinas et al., 2008). These intracellular events, leading to the phosphorylation of AMPA receptors and cAMP response element binding protein (CREB; Roberson et al., 1999; Ahmed and Frey, 2005), can ultimately result in downstream mRNA transcription and subsequent protein synthesis, processes critical for the initiation of enduring forms of synaptic plasticity(Kandel and Squire, 2000; Miyamoto, 2006; Abraham and Williams, 2008; also see Kleschevnikov and Routtenberg, 2001; Emptage et al., 2003 for discussions of presynaptic mechanisms in LTP maintenance, including protein kinase $\mathrm{C}$ and changes in presynaptic transmitter release). The unifying theme of a critical role of postsynaptic-NMDARCa2+-dependent mechanisms demonstrated for HFS-induced LTP in the CA1 area extends to LTP in extra-hippocampal brain systems, including amygdala and neocortex (Huang and Kandel, 1998; Blair et al., 2001; Heynen and Bear, 2001; Speechley et al., 2007).

\subsection{From high to low: Have HFS protocols met their match?}

It is clear that HFS or TBS provide effective means to induce classical, NMDARdependent LTP. In contrast, protocols employing low frequency stimulation (LFS) typically result in minimal or no LTP, or in long-term depression (LTD) of hippocampal synapses (Bramham and 
Srebro, 1987; Christie and Abraham, 1992; Christie et al., 1994; Thiels et al., 1994; Wasling et al., 2002; Massey and Bashir, 2007). However, a series of recent reports throws into question whether the "HFS-LTP, LFS-LTD" dichotomy fully captures the entire spectrum and directions of synaptic modifications that are expressed at hippocampal and some extra-hippocampal synapses (see Table 1 for summary of findings). In two initial reports, Li et al. $(1998,2001)$ characterized a surprising form of synaptic potentiation following LFS of inputs to the amygdala. Their studies revealed that, in amygdala slices, prolonged LFS ( $1 \mathrm{~Hz}, 15 \mathrm{~min})$ of fibers in the external capsule (EC) elicits a slow onset, gradually progressing enhancement of excitatory postsynaptic potential (EPSP) amplitude in basolateral amygdala neurons, which persisted for over 30 min following delivery of the LFS protocol (Li et al., 1998; 2001). More recently, Huang and Kandel (2007) examined synaptic potentiation induced by LFS in the cortical-lateral amygdala (LA) pathway in vitro, confirming that LFS can elicit gradually developing synaptic potentiation in the LA that reached stable levels at approximately 2-3 $\mathrm{h}$ after induction (Huang and Kandel, 2007). Importantly, LFS-induced LTP (LFS-LTP) is not restricted to corticalamygdala synapses, as similar phenomena have now been characterized at some hippocampal synapses. Michel Vignes and his colleagues (Lanté et al., 2006 a, b) demonstrate that application of LFS ( $1 \mathrm{~Hz}, 5 \mathrm{~min})$ to area CA1 produces a gradual, slow-onset form of potentiation of CA1 synapses in vitro, lasting for at least $30 \mathrm{~min}$ post induction. Interestingly, this form of LFS-LTP appears to be specific to the CA1 field, since the same LFS protocol did not elicit potentiation when applied to either CA3 or the dentate gyrus (Lanté et al., 2006 a,b). Together, these highly counterintuitive data sets were the first to demonstrate the phenomenon of high frequencyindependent enhancement of synaptic strength in the forebrain. 
Table 1. Summary of mechanisms underlying low frequency stimulation (LFS)-induced long-lasting synaptic potentiation in the hippocampus and amygdala.

EC (external capsule), LA (lateral amygdala), mGluR5 (metabotropic glutamate receptor subtype 5), NMDA (N-methyl-D-aspartic acid), GluR5 (glutamate receptor 5), 5-HT4 (5-

hydroxytryptamine receptor 4), D1 (dopamine receptor subtype 1), $\beta$-adrenergic (beta-adrenergic receptor), $\mathrm{Ca}^{2+}$ (calcium), cAMP (cyclic adenosine monophosphate), PKA (protein kinase A), MAP (Mitogen-activated protein).

\begin{tabular}{|c|c|c|c|}
\hline Anatomical Region & $\begin{array}{c}\text { Stimulation } \\
\text { Protocol }\end{array}$ & Membrane Receptors & $\begin{array}{c}\text { Second } \\
\text { Messengers }\end{array}$ \\
\hline $\begin{array}{l}\text { CA1 (Lanté et al. } 2006 \\
\text { a,b) }\end{array}$ & $1 \mathrm{~Hz}, 3-5 \mathrm{~min}$ & $\begin{array}{l}\text { mGluR 5-dependent } \\
\text { NMDA-independent }\end{array}$ & $\begin{array}{l}\text { Rise in } \\
\text { intracellular } \mathrm{Ca}^{2+} \\
\rightarrow \text { cAMP } \rightarrow \text { PKA } \\
\text { MAP } \\
\text { independent }\end{array}$ \\
\hline $\begin{array}{l}\text { CA1 (Habib \& } \\
\text { Dringenberg, in press) }\end{array}$ & $\begin{array}{l}1 \mathrm{~Hz}(1: 40 \mathrm{~min}) \\
\text { (alternating MS } \\
\text { and } \mathrm{CA} 3 \mathrm{stim} \text {, } \\
0.5 \mathrm{~Hz} \text { each) }\end{array}$ & $\begin{array}{l}\text { NMDA-dependent } \\
\text { nicotinic or muscarinic } \\
\text { independent }\end{array}$ & Unknown \\
\hline $\begin{array}{l}\text { EC-LA pathway (Li et } \\
\text { al., 2001) }\end{array}$ & $1 \mathrm{~Hz}, 15 \min$ & $\begin{array}{l}\text { GluR5-dependent } \\
\text { NMDA-independent }\end{array}$ & $\mathrm{Ca}^{2+}$ influx \\
\hline $\begin{array}{l}\text { EC-LA pathway ( Huang } \\
\text { \& Kandel, 2007) }\end{array}$ & $1 \mathrm{~Hz}, 15 \mathrm{~min}$ & $\begin{array}{l}\text { 5-HT4, D1 and } \beta- \\
\text { adrenergic dependent } \\
\text { NMDA-independent }\end{array}$ & $\begin{array}{l}\text { PKA } \rightarrow \text { new } \\
\text { protein synthesis }\end{array}$ \\
\hline
\end{tabular}


The different form LFS-LTP at synapses between Schaffer collateral/commissural fibers and CA1 neurons has recently been characterized under in vivo conditions using urethane anesthetized preparations. Habib and Dringenberg (2009), using alternating single pulse stimulation of the medial septum (MS) and CA3 (50 pulses each at $0.5 \mathrm{~Hz}, 1000 \mathrm{~ms}$ interstimulus interval [ISI]], noted a gradual increase of fEPSP amplitude recorded in both strata radiatum and oriens of CA1. Many of the characteristics of this type of in vivo synaptic potentiation elicited by low-frequency, alternating MS- hippocampal stimulation (MS-H LTP) mirror those of classical HFS-LTP, including the long-lasting maintenance phase $(>4 \mathrm{~h})$, the effectiveness of repeated induction episodes to result in saturation of synaptic strengthening, and its dependence on NMDA receptor activation, as discussed below.

In toto, the evidence summarized here provides initial, but compelling evidence for the existence of forms of long-lasting synaptic potentiation that do not require classic, high-frequency parameters for their effective induction in hippocampal and extra- hippocampal systems.

\subsection{HFS-LTP vs. LFS-LTP mechanisms: How similar are they?}

The studies reviewed above provide evidence that some forms of LFS-induced synaptic potentiation rely on mechanisms quite distinct from those known to mediate classical HFS-LTP. Remarkably, the LFS-induced enhancement of fEPSP amplitude characterized in CA1 by Lanté et al. $(2006 \mathrm{a}, \mathrm{b})$ is independent of NMDA receptors, but appears to involve glutamate binding to mGluR5 receptors, the release of $\mathrm{Ca} 2+$ from internal stores, and PKA activation. Similarly, LFSinduced synaptic facilitation in lateral and basolateral amygdala neurons is independent of NMDA-receptors (Li et al., 2001; Huang and Kandel, 2007). Rather, potentiation in the basolateral amygdala requires activation of kainate-type ionotropic glutamate receptors (GluR5; 
Li et al., 2001), while LF-induced long-lasting synaptic enhancement in the EC-LA pathway depends on co- activation of several neuromodulatory receptor types, including serotonin 5-HT4, dopamine D1 and $\beta$-adrenergic receptors (Huang and Kandel, 2007). In addition, Huang and Kandel (2007) established that their form of LFS induced plasticity requires PKA activation and the synthesis of new proteins, similar to mechanisms mediating the long- lasting maintenance of classical LTP at amygdaloid and other synapses (Huang et al., 2000; Okulski et al., 2002; Huang and Kandel, 2007). It will be of interest to determine whether mechanisms of LFS-induced synaptic enhancement show parallels to forms of HFS-induced LTP that are independent of NMDARs but require opening of voltage-gated calcium channels for their induction (e.g., mossy fiber-CA3 synapses; Fisher and Johnston, 1990; Jaffe and Johnston, 1990; Nicoll and Schmitz, 2005).

The fact that several forms LFS-LTP are independent of NMDAR activation is, of course, surprising, in light of the paramount role of this receptor as coincidence-detection of preand post-synaptic activity and induction of numerous forms of synaptic plasticity. Consequently, the tight temporal constraints governing NMDA-dependent, coincidence-based plasticity may not apply to LFS-LTP, explaining the effectiveness of LFS to induce synaptic enhancement. On the other hand, intracellular mechanisms engaged by HFS and LFS appear to share commonalities, with a central role played by free, intracellular $\mathrm{Ca}^{2+}$ as a final, common signal triggering further, downstream changes.

Contrary to the mechanisms of LFS-induced synaptic enhancement in CA1 and amygdala in vitro, synaptic enhancement in CA1 elicited by alternating LFS of commissural and medial septal afferents in vivo does not occur in the presence of local APV application, indicative of a critical role of NMDAR activation (Habib and Dringenberg, 2009). Surprisingly, even though the 
MS provides the major cholinergic input to the hippocampal formation, MS-H LTP does not appear to require cholinergic (muscarinic and nicotinic) receptor activation. Whether other neuromodulatory signals are involved in this type of plasticity has not been examined (see Huang and Kandel, 2007).

The difference regarding the role of NMDAR-activation for LFS-LTP in CA1 seen by Dringenberg and Habib (2009) and Lanté et al. (2006 a,b) may indicate the existence of several, fundamentally different forms of LFS-induced synaptic enhancement. Further, the fact that some forms of LFS-LTP appear to be NMDAR- dependent is counterintuitive, given that afferent stimulation at a frequency of $1 \mathrm{~Hz}$ is well beyond the time window thought to be effective in eliciting NMDA-dependent potentiation (Bennett, 2000; Dan and Poo, 2004; Malenka and Bear, 2004; Bi and Rubin, 2005; Yao and Dan, 2005; see also Massey and Bashir, 2007). It is noteworthy, however, that some forms of NMDA-dependent potentiation exhibit surprisingly wide temporal windows for the integration of synaptic inputs to trigger synaptic enhancement (Popescu et al., 2007). Whether or not the alternating single pulse stimulation to MS and Schaffer collaterals/commissural fibers triggers intracellular mechanisms similar to classical LTP or other forms of LFS-induced potentiation is currently unknown.

\subsection{LFS-LTP: Relevance to endogenous hippocampal activity}

A prominent feature of hippocampal activity is the prevalence of several, distinct oscillatory patterns that are tightly linked to specific behavioral states and exert important influences over the expression of synaptic plasticity (Buzsáki, 2002; Hyman et al., 2003; Axmacher et al., 2006). Clearly, induction protocols used to initiate modifications of hippocampal synaptic efficacy should mimic activity patterns present in the hippocampus and/or its major afferents systems. For example, TBS, a highly effective LTP induction protocol (Larson et al., 
1986; Stäubli \& Lynch, 1987), mimics theta (3-12 Hz) activity present in the hippocampus during specific types of behavior (walking, postural adjustments, head movements, REM sleep, "Type 1 behavior"; Vanderwolf, 1969, 1988; Buzsáki, 2002). However, other prominent hippocampal oscillatory patterns occur at frequencies significantly below the theta range. Large amplitude, irregular activity (LIA) during behavior such as immobility, grooming, licking, or slow wave sleep (“Type 2 behavior”, Vanderwolf, 1969, 1988) contains sharp wave complexes, events of significant cellular excitation that are repeated at frequencies between 0.02 to $3 \mathrm{~Hz}$ (Buzsáki, 1989). Further, a highly regular, slow oscillatory pattern of $\sim 1 \mathrm{~Hz}$ has recently been characterized in the hippocampus of sleeping and anesthetized rats (Wolansky et al. 2006; Schall et al., 2008), activity that parallels the slow, neocortical oscillations present in slow wave sleep (Steriade et al., 1993). Importantly, these endogenous activity patterns occur at frequencies overlapping with those used for LFS protocols in the studies summarized above. Consequently, it is tempting to ask whether the mechanisms underlying LFS-LTP are of relevance to those mediating the influence of slow oscillations on the encoding, consolidation, and transfer of memory traces in hippocampal and neocortical ensembles.

The role of the hippocampus in memory-related functions is not only engaged during wakefulness and information acquisition, but also during sleep and related consolidation processes whereby memory traces are believed to be transferred to specific neocortical areas (Buzsáki, 1989; McGaugh, 2000). A prominent hypothesis suggests that rhythmic population synchrony serves as a mechanism to promote plasticity and memory consolidation during slow wave sleep (SWS; Buzsáki, 1989; Sipas and Wilson, 1998; Wolansky et al., 2006). Remarkably, a potentially causal relationship between slow rhythms and memory consolidation in neocortical and hippocampal networks is gradually emerging (Huber et al., 2004; Peigneux et al., 2004; 
Marshall et al., 2006; Hoffman et al., 2007). For example, performance on an implicit motor task in humans improved during sleep, an effect that correlated with an increase in power of slow wave activity $(<4 \mathrm{~Hz})$, which was restricted to the location of cortical networks known to mediate visual-motor learning (Huber et al., 2004). Even more impressively, transcranial magnetic stimulation of humans after the onset of stage II sleep can improve performance on a declarative task, an effect that occurred with stimulation at a frequency of $0.75 \mathrm{~Hz}$, while theta frequencies were ineffective (Marshall et al., 2006). Clearly, these studies support the notion that slow oscillatory patterns have an important, possibly causal role in synaptic changes that serve to consolidate specific types of memories during SWS. The fact that replay of cellular activity from waking to subsequent SWS occurs predominantly in phase with low-frequency activity patterns (i.e., during hippocampal sharp waves; Kudrimoti et al., 1999) is consistent with this hypothesis.

\subsection{Next steps: The future of LFS-LTP}

Evidently, the phenomenon of LFS-induced synaptic potentiation and the cellular and molecular mechanisms mediating this form of plasticity require a much more complete characterization. It appears that some commonalities exist among different types of LFS-induced synaptic potentiation, especially with regard to the role of intracellular $\mathrm{Ca}^{2+}$ as a final, common signal triggering further, downstream changes. At the same time, important differences have been reported regarding some mechanisms underlying LFS-induced potentiation (e.g., NMDAdependent and independent). This might suggest the existence of distinct types of LFS-induced plasticity, similar to HFS- induced LTP, an assumption that requires assessment based on a thorough and systematic analysis of the various receptor and intracellular mechanisms engaged in different types of LFS plasticity. Also, given that the majority of studies to date have used in vitro preparations, it is imperative to examine and characterize this phenomenon in the intact brain. 
In addition to mechanistic studies, deciphering the functional, behavioral significance of LFS-induced synaptic potentiation remains a major challenge. As discussed, the frequency bands of slow rhythmic activity thought to underlie memory consolidation closely correspond to the range of frequencies used to elicit LFS-induced LTP in the hippocampus and amygdala, suggesting that LFS-LTP may serve as cellular mechanism for learning and state-dependent memory consolidation. A critical test of this hypothesis will require an integrative approach using intact, in vivo preparations, allowing the inclusion of behavioral assessments to establish more direct links between synaptic changes and the process of memory consolidation. In this regard, rodent models will serve as a critical bridge between in vitro slice work and the literature on slow-wave oscillations and sleep-dependent consolidation in humans. Ultimately, work on LFSLTP may provide a platform for understanding the cellular processes mediating the influence of endogenous, low frequency oscillations on plasticity in relation to memory consolidation and transfer between hippocampal and neocortical networks.

\subsection{Conclusions}

Since the discovery of LTP, application of HFS of glutamatergic synapses has provided a highly effective method for eliciting long-lasting increases in synaptic efficacy, while lowfrequency activity patterns are assumed to produce synaptic depression at hippocampal and extrahippocampal synapses. Here, we have summarized evidence for the existence of high frequencyindependent forms of synaptic potentiation, some of which may depend on cellular and molecular mechanism distinct from those mediating classical LTP. Clearly, extensive studies are needed to mechanistically characterize LFS-induced potentiation, and decipher its functional roles in memory consolidation. Given the increased recognition that low frequency neural events play a critical role in consolidation processes, we anticipate that the emerging field of LFS-LTP will 
bring an important paradigm shift to the study of plasticity processes in relation to learning and memory functions in the mammalian forebrain. 


\section{Chapter 4. Surprising similarity between mechanisms mediating low (1 Hz)-and high $(100 \mathrm{~Hz})$-induced long-lasting synaptic potentiation in \\ CA1 of the intact hippocampus}

As published in "Surprising similarity between mechanisms mediating low (1 Hz)-and high (100 Hz)induced long-lasting synaptic potentiation in CAl of the intact hippocampus " by Habib, D \& Dringenberg, HC. 2010. Neuroscience Vol. 170, 489-496.

\subsection{Abstract}

It is generally assumed that long lasting synaptic potentiation (long-term potentiation, LTP) and depression (long-term depression, LTD) result from distinct patterns of afferent activity, with high and low frequency activity favoring LTP and LTD, respectively. However, a novel form of N-methyl-D-aspartate (NMDA) receptor-dependent synaptic potentiation in the hippocampal CA1 area in vivo induced by low frequency afferent stimulation has recently been demonstrated. Here, we further characterize the mechanisms mediating this low frequency stimulation (LFS)induced LTP in area CA1 of intact, urethane-anesthetized preparations. Consistent with previous reports, alternating, low frequency $(1 \mathrm{~Hz})$ stimulation of CA1 afferents originating in the contralateral CA3 area and the medial septum resulted in gradually developing, long lasting $(>2$ hours) LTP of field excitatory postsynaptic potentials (fEPSPs) recorded in CA1. Local application of the protein synthesis inhibitor anisomycin in CA1 blocked LFS-induced LTP, as did application of H89, an inhibitor of protein kinase A. Given the apparent overlap in molecular mechanisms mediating LFS-LTP and "classic" high-frequency stimulation (HFS)-induced LTP in 
CA1, we examined the relation between these forms of LTP by means of occlusion experiments. LFS, delivered to synapses saturated by initial HFS, resulted in a gradually developing LTD, rather than the normally seen LTP. Conversely, initial induction of LFS-LTP reduced the amount of subsequent HFS-LTP. Together, these experiments reveal a surprising similarity in the molecular mechanisms (dependence on NMDA receptors, protein kinase A, protein synthesis) mediating LTP induced by highly distinct (1 vs. $100 \mathrm{~Hz}$ ) induction protocols. Importantly, these findings further challenge the "high-frequency-LTP, low-frequency LTD" dogma by demonstrating that this dichotomy does not account for all types of plasticity phenomena at central synapses. 


\subsection{Introduction}

It is generally accepted that high-frequency stimulation (HFS) of glutamatergic fibers provides an effective method for eliciting long-lasting increases in synaptic efficacy (e.g., longterm potentiation, LTP) while low-frequency stimulation (LFS) protocols typically induce longterm depression (LTD; Bliss and Lómo, 1973; Raymond, 2007; Caporale and Dan, 2008). Surprisingly, however, several reports have now confirmed the existence of LFS-induced synaptic potentiation (LFS-LTP) in the amygdala and hippocampal formation (Mayford et al., 1995; Thomas et al., 1996; Li et al., 1998; Lanté et al., 2006a, b; Huang and Kandel, 2007; Habib and Dringenberg, 2009). In the hippocampal CA1 area in vitro, $1 \mathrm{~Hz}$ stimulation of local CA1 synapses results in a gradually developing, relatively stable ( $>30 \mathrm{~min})$ potentiation of field excitatory postsynaptic potentials (fEPSPs; Lanté et al., 2006a, b). This form of LFS-LTP is independent of N-methyl-D-aspartic (NMDA) receptors and requires mGluR5 activation, the release of $\mathrm{Ca}^{2+}$ from internal stores, and protein kinase A (PKA; Lanté et al., 2006b). Similarly, in the lateral and basolateral amygdala, NMDA receptor-independent LTP can occur following $1 \mathrm{~Hz}$ stimulation of the external capsule in vitro, a phenomenon mediated by several mechanisms, including GluR5, several neuromodulatory receptors (e.g., 5-HT4, D1), as well as PKA activation and protein synthesis (Li et al., 2001; Huang and Kandel, 2007).

Recent evidence has extended this work by demonstrating similar phenomena in the intact brain. In anesthetized rats, fEPSPs in area CA1 show significant, long lasting potentiation (> $5 \mathrm{~h}$ ) following alternating, single pulse stimulation (at $1 \mathrm{~Hz}$ ) of the medial septum (MS) and hippocampal (H) CA3 area (MS-H LTP; Habib and Dringenberg, 2009). In contrast to the forms of LFS-LTP noted in vitro, MS-H LTP requires NMDA receptor activation, similar to most types of classical, HFS-LTP. Consequently, it is possible that several types of LFS-LTP exist at 
hippocampal and other synapses, distinguished by differences in receptor and cellular signals required for their induction. At present, the intracellular mechanisms mediating MS-H LTP have not been elucidated. Consequently, the present experiments aimed to further characterize some of the critical signaling mechanisms mediating this novel form of plasticity in the intact brain preparation. Further, the relation between MS-H LTP and LTP induced by high-frequency (100 $\mathrm{Hz}$ ) stimulation was assessed by conducting occlusion experiments, delivering LFS either before or after HFS stimulation of CA1 afferents. The potential role of low frequency, oscillatory activity patterns in sleep-related memory consolidation has received considerable attention in the last several years (Buzsáki, 1998; Sirota and Buzsáki, 2005; Born et al., 2006). It is possible that the form of LTP characterized here could provide one of the mechanisms mediating the influence of endogenous slow oscillations on synaptic plasticity, thought to occur during sleep in the mammalian brain.

\subsection{Experimental Procedures}

\subsubsection{Subjects and surgical preparation}

All experiments were performed on male Long-Evans rats (320-450 g) housed in a colony room (12/12-h reverse light cycle) with food and water available ad libitum. The procedures were conducted in accordance with the guidelines of the Canadian Council on Animal Care and approved by the Queen's University Animal Care Committee. Each rat was used for only one experiment.

Experimental procedures were conducted under deep urethane anaesthesia $(1.5 \mathrm{~g} / \mathrm{kg}$, intraperitoneal [i.p.], administered in three $0.5 \mathrm{~g} / \mathrm{kg}$ doses every $20 \mathrm{~min}$ and supplemented with $0.2-0.6 \mathrm{~g} / \mathrm{kg}$ as required prior to the onset of data collection). Rats were placed in a stereotaxic apparatus and body temperature was maintained between 36 and $37^{\circ} \mathrm{C}$ by means of an electrical 
heating blanket. An incision was made to expose the skull surface and small skull holes were drilled above the CA3 region (AP -4.16, ML +3.0, V -4.0), the contralateral CA1 (stratum radiatum) area (AP -4.16, ML -3.0, $\mathrm{V}-3.0$ ), and at bregma point (AP 0.0, ML 0.0, V - 6.5) to

gain access to the MS. Skull holes for ground and reference electrodes (jewellery screws attached to miniature connectors) were made in the bone overlying the prefrontal cortex and cerebellum, respectively. An additional skull hole for a MS stimulation return electrode (jewellery screw) was drilled adjacent to bregma. All stereotaxic measurements were based on the anatomical work of Paxinos and Watson (1998). Final ventral placements of the CA3 stimulation and the CA1 recording electrode were adjusted to elicit maximal fEPSP amplitude and paired-pulse facilitation (100 ms interstimulus interval, ISI) in area CA1 in response to contralateral CA3 stimulation.

\subsubsection{Electrophysiology}

Stimulation of CA3 $(0.2 \mathrm{~ms}$ pulses every $30 \mathrm{~s}$, intensity adjusted to yield $50-60 \%$ of maximal fEPSP amplitude) was provided by a concentric bipolar electrode (Rhodes Medical Instruments Series 100, David Kopf, Tujunga, CA, USA) connected to a stimulus isolation unit providing a constant current output (PowerLab/16 s system with ML 180 Stimulus Isolator, AD Instruments, Toronto, Canada). MS stimulation was provided through a monopolar electrode (Teflon insulated stainless steel, $125-\mu \mathrm{m}$ tip diameter) delivering single, negative constant-current pulses (0.2 ms duration, $0.5 \mathrm{~mA})$.

All fEPSPs in stratum radiatum of CA1 were differentially recorded (Teflon insulated stainless steel wire, $125-\mu \mathrm{m}$ tip diameter) against a reference electrode placed in the bone overlaying the cerebellum. The CA1 signals were amplified, filtered $(0.3-1 \mathrm{kHz})$, digitized (10 $\mathrm{kHz}$ ), and stored for subsequent off-line analysis using the PowerLab system running Scope software (v. 4.0.2). 


\subsubsection{Data collection and pharmacological manipulation}

Prior to formal data collection, input-output curves were established for each animal by recording fEPSPs in CA1 in response to CA3 stimulation between 0.1 and $1.0 \mathrm{~mA}(0.1 \mathrm{~mA}$ increments). Based on these input-output curves, a stimulation intensity eliciting between $50-60 \%$ of the maximal fEPSP amplitude was chosen for the subsequent experiment. For each rat, 60 initial baseline fEPSPs (every $30 \mathrm{~s}$ ) were recorded. For all animals receiving local drug application in area CA1, a second baseline recording of 60 fEPSPs (every $30 \mathrm{~s}$ ) was taken after

drug application to verify that the drug did not alter baseline synaptic strength at CA3-CA1 synapses.

Following stable baseline recordings, the MS-CA3 stimulation protocol was delivered, consisting of 100 single pulses, alternating between MS and CA3 (50 pulses for each area, delivered at $0.5 \mathrm{~Hz}$, with an ISI of $1000 \mathrm{~ms}$ between successive, alternating MS and CA3 pulses). Typically, recordings of fEPSPs in CA1 (every $30 \mathrm{~s}$, as in baseline) in response to single pulse CA3 stimulation continued for four hours following the alternating stimulation protocol. In one group of rats, the MS-CA3 stimulation protocol was delivered twice, with the second delivery occurring $2 \mathrm{~h}$ after the initial MS-CA3 stimulation. Further, to verify that alternating co-activation of both MS and CA3 fibers is required to elicit LFS-induced potentiation, a group of control animals was studied that received only CA3 pulses during the induction protocol (100 pulses at 1 $\mathrm{Hz})$.

In order to assess the relation between MS-H LTP and LTP induced by HFS, occlusion experiments were conducted by delivering the MS-CA3 protocol either before or after HFS (100 $\mathrm{Hz}$ ) stimulation of CA3-CA1 fibers. One group of animals received initial HFS of CA3 (100 pulses at $100 \mathrm{~Hz}$ ), repeated at $10 \mathrm{~min}$ intervals until LTP saturation was reached (i.e., no further 
fEPSP amplitude enhancement in CA1 was seen), at which point the MS-CA3 stimulation protocol was delivered. Another group received the MS-CA3 stimulation protocol first, followed $2 \mathrm{~h}$ later by HFS (as above), which again was repeated (every $10 \mathrm{~min}$ ) until a saturation point was reached. In order to assess the effects of initial saturation of MS-H LTP on subsequent HFSinduced LTP, an additional group of animals received repeated episodes of MS-CA3 stimulation at $2 \mathrm{~h}$ intervals until fEPSP amplitude in CA1 could no longer be potentiated with this LFS. Following LFS-induced saturation, these animals received repeated episodes of HFS (every 10 min, same as above) until a saturation point was reached.

For experiments assessing the role of protein synthesis and PKA, a microdialysis probe (Mab. 2.14.2; 2-mm active polyethersulfane membrane length, 35-kDa cutoff; S.P.E. Ltd., North York, ON, Canada) was placed immediately adjacent to the recording electrode in CA1, with the probe tip extending about $1 \mathrm{~mm}$ below the electrode tip, permitting local drug application in CA1 using reverse microdialysis. The dialysis probe was connected to a Hamilton microsyringe driven by a microdialysis pump (CMA/102, CMA Microdialysis, Solna, Sweden) and continuously perfused (1.0 $\mu \mathrm{L} / \mathrm{min})$ with a mixture of artificial cerebrospinal fluid (aCSF) and dimethyl sulfoxide (DMSO; aCSF/DMSO ratio of 9:1; aCSF composition in mM: $118.3 \mathrm{NaCl}, 4.7 \mathrm{KCl}, 1.2$ MgSO4, 1.2 NaH2PO4, 2.5 CaCl2, 10.0 glucose, 22.1 NaHCO3). Reverse dialysis allows local drug application by means of concentration-driven diffusion of drug molecules through the dialysis probe membrane into the surrounding neural tissue, with estimated drug concentrations outside the membrane reaching approximately $10 \%$ of concentration contained in the perfusion medium (e.g. Oldford and Castro-Alamancos, 2003).

For these experiments, an initial baseline (30 $\mathrm{min})$ was obtained prior to drug application (i.e., during aCSF + DSMO application). Subsequently, either the transcription inhibitor 
anisomycin $(0.5$ and $1.0 \mathrm{mM})$ or the selective PKA inhibitor $\mathrm{H} 89(1.0 \mathrm{mM})$ was applied in CA1 and continued throughout the rest of the experiment. A second $(30 \mathrm{~min})$ baseline in the presence of the drug was obtained, starting at 10 min following the onset of drug application. After this baseline, the MS-CA3 alternating stimulation protocol was delivered and recordings continued for 4 hours, as described above. Control animals received continuous aCSF + DMSO during the course of the entire experiment. In addition, for both drugs and drug concentrations, further control experiments without the delivery of the MS-CA3 protocol were also performed to assess the effects of long-lasting drug application on baseline, non-potentiated synaptic strength of CA3-

\section{CA1 synapses.}

At the end of each experiment, rats were perfused through the heart with $10 \%$ formalin, their brains were extracted, and standard histological techniques were used to verify all electrode placements. Data obtained with inaccurate placements were excluded from the data analysis.

\section{Data Analysis}

All data are expressed as a mean \pm standard error of mean (S.E.M.). The maximal fEPSP amplitude was analyzed offline by the Scope software. Subsequently, amplitude data were averaged over 10-min intervals, and these averages were normalized by dividing all data for each rat by the average baseline (pre MS-CA3 alternating stimulation) amplitude of that animal. For statistical analyses, analyses of variance (ANOVA) and, where statistically appropriate, simple effects tests were computed using the software package CLR ANOVA (v.1.1, Clear Lake Research Inc., Houston, TX). 


\subsection{Results}

\subsubsection{Synaptic enhancement following alternating MS-CA3 single pulse stimulation (MS-H LTP)}

In urethane-anesthetized rats, single stimulation pulses applied to area CA3 produced positive-going fEPSPs (latency to peak about 10-11 ms) recorded in the stratum radiatum of the contralateral CA1 area (Fig. 7 insert). A group of control animals $(n=5)$ received only CA3 stimulation during the induction protocol (i.e., 100 single pulses to CA3 at $1 \mathrm{~Hz}$ for each induction episode). In these rats, fEPSP amplitude in area CA1 did not show significant changes during the course of the experiment (Fig. 7), with mean amplitude of $95 \%$ following the first induction protocol (values are averages over $30 \mathrm{~min}$ of recording between $1.5-2 \mathrm{~h}$ after protocol delivery), and $89 \%$ during the epoch occurring 1.5-2 h after the second protocol delivery. In contrast, delivery of the protocol consisting of alternating single pulses applied to the MS and CA3 $(n=7)$ produced a gradual, significant increase in fEPSP amplitude over the course of the experiment (Fig. 7; MS-CA3 stimulation consisted of a total of 50 pulses at $0.5 \mathrm{~Hz}$ for each area, starting with MS, $1000 \mathrm{~ms}$ ISI). Amplitude of fEPSPs averaged over the 1.5 to $2 \mathrm{~h}$ epochs after delivery of the first and second induction episode were $113 \%$ and $129 \%$ of baseline, respectively (Fig. 7). Thus, in accordance with previous data (Habib and Dringenberg, 2009), alternating coactivation of MS and CA3 fibers elicits LTP, while the same total number of pulses applied to only CA3 fails to elicit significant potentiation.

\subsubsection{MS-H LTP is dependent on protein synthesis and PKA activation}

Long-lasting synaptic potentiation generally requires activation of protein kinases and the subsequent synthesis of new proteins (Kandel and Squire, 2000; Miyamoto, 2006; Abraham and 


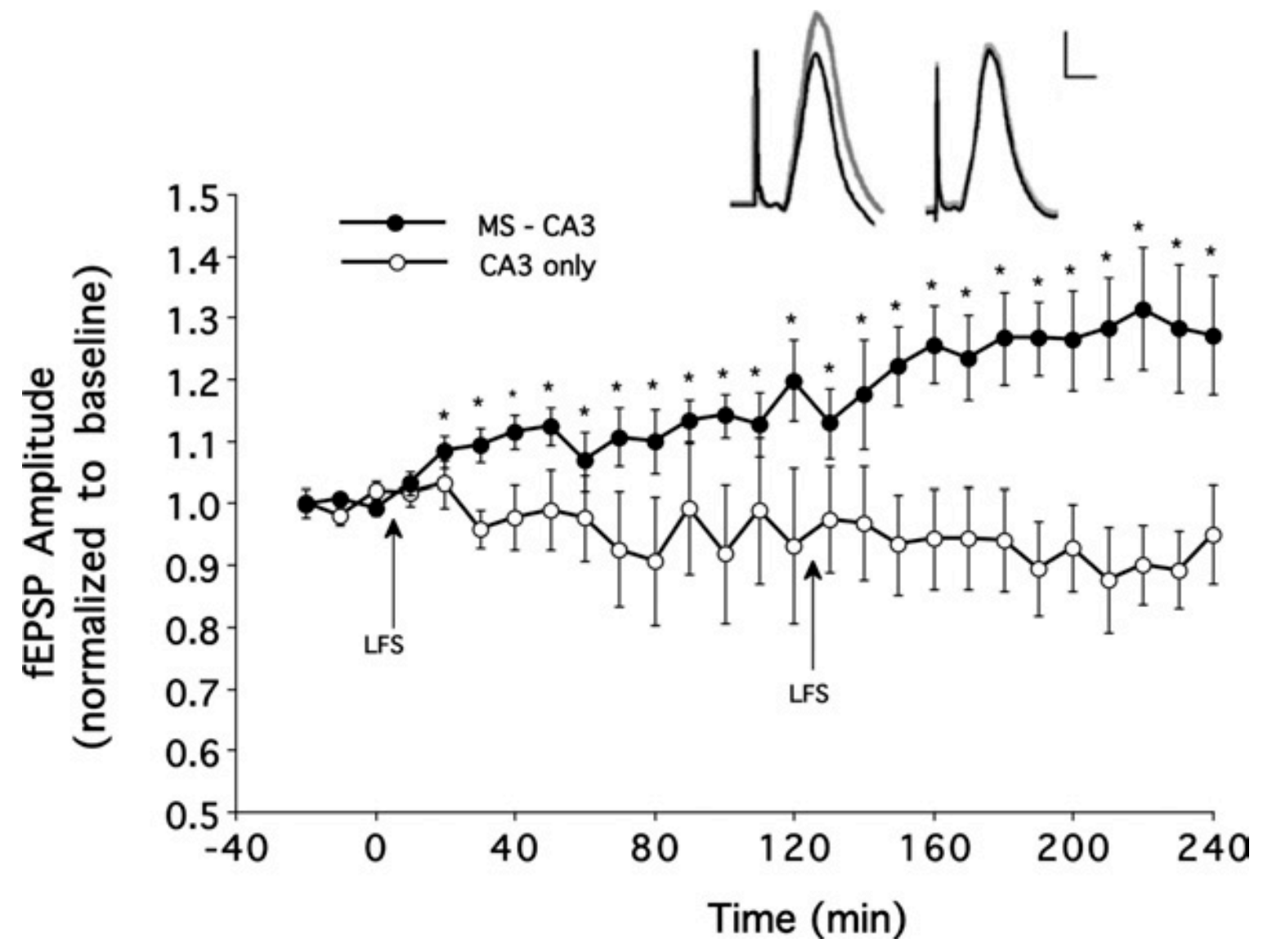


Figure 7. The effect of alternating stimulation of the medial septum (MS) and CA3 area on amplitude of CA1 field excitatory postsynaptic potentials (fEPSPs) in the stratum radiatum. Delivery of alternating, low frequency stimulation (LFS, at arrows) of the MS and CA3 (MSCA3; $n=7$; two induction episodes delivered $2 \mathrm{~h}$ apart) resulted in a significant enhancement of fEPSP amplitude. In contrast, single pulses applied to CA3 only (i.e., in the absence of MS stimulation) failed to induce synaptic enhancement $(n=5)$. Inserts depict typical fEPSPs in stratum radiatum during baseline (black) and at the end of the experiment (grey) in animals receiving either MS-CA3 (left) or only CA3 stimulation (right; each fEPSP shown is an average of 10 individual sweeps, calibration is $10 \mathrm{~ms}$ and $1 \mathrm{mV}$ ). Statistics: ANOVA comparing MS-CA3 and CA3 only groups, effect of group, $F_{1,10}=13.5, P=0.0042$, effect of time, $F_{26,260}=1.8, P=$ 0.01 ; group by time interaction, $F_{26,260}=5.2, P<0.0001$; simple effect of time significant for animals receiving MS-CA3 stimulation $\left(F_{26,156}=5.7, P<0.0001\right)$, but not for animals receiving only CA3 stimulation $\left(F_{26,104}=0.7, P=0.82\right) . *$ denote significant $(P<0.05 ;$ simple effects tests) group differences. 
Williams, 2008). Consequently, we assessed whether these mechanisms mediate LFS-induced plasticity studied under in vivo conditions. For these experiments, local drug application at the CA1 recording site was performed by means of reverse microdialysis. In the presence of continuous aCSF + DMSO application $(n=8)$, alternating MS-CA3 stimulation produced a gradual, significant increase of fEPSP amplitude over the $4 \mathrm{~h}$ recording period following protocol delivery (Fig. 8A; average fEPSP amplitude of $122 \%$ of baseline during the final $30 \mathrm{~min}$ of the experiment).

For the initial experiments assessing the effects of protein synthesis inhibition, anisomycin at a concentration of $1 \mathrm{mM}$ completed blocked MS-H LTP induced by alternating MS-CA3 stimulation ( $n=7$; data not shown). However, this concentration also appeared to suppress non-potentiated fEPSP amplitude ( $n=4$; data not shown). Consequently, these experiments were repeated with a lower anisomycin concentration $(0.5 \mathrm{mM}, n=6)$, which produced only a minor, non-significant change in non-potentiated fEPSPs (Fig. 8C; $n=5$, fEPSP amplitude of $88 \%$ of baseline during the last $30 \mathrm{~min}$ of the experiment). In the presence of this lower concentration of anisomycin, MS-CA3 stimulation $(n=6)$ failed to elicit synaptic potentiation and, in fact, resulted in a significant fEPSP depression (Fig 8A; average fEPSP amplitude of $49 \%$ of baseline during the final $30 \mathrm{~min}$ of the experiment). This suppressant effect was due to the delivery of the stimulation protocol, since the decrease in fEPSP amplitude in MSCA3 rats was significantly greater than that seen in control animals exposed to anisomycin without MS-CA3 stimulation (Fig. 8C; $n=5$ ).

Next, we probed the potential role of PKA in MS-H LTP by applying H89 (1 mM, $n=8)$, a selective inhibitor of cAMP-dependent PKA. In the presence of H89, delivery of the MS-CA3 protocol no longer induced significant levels of synaptic potentiation (Fig. 8B; final fEPSP 

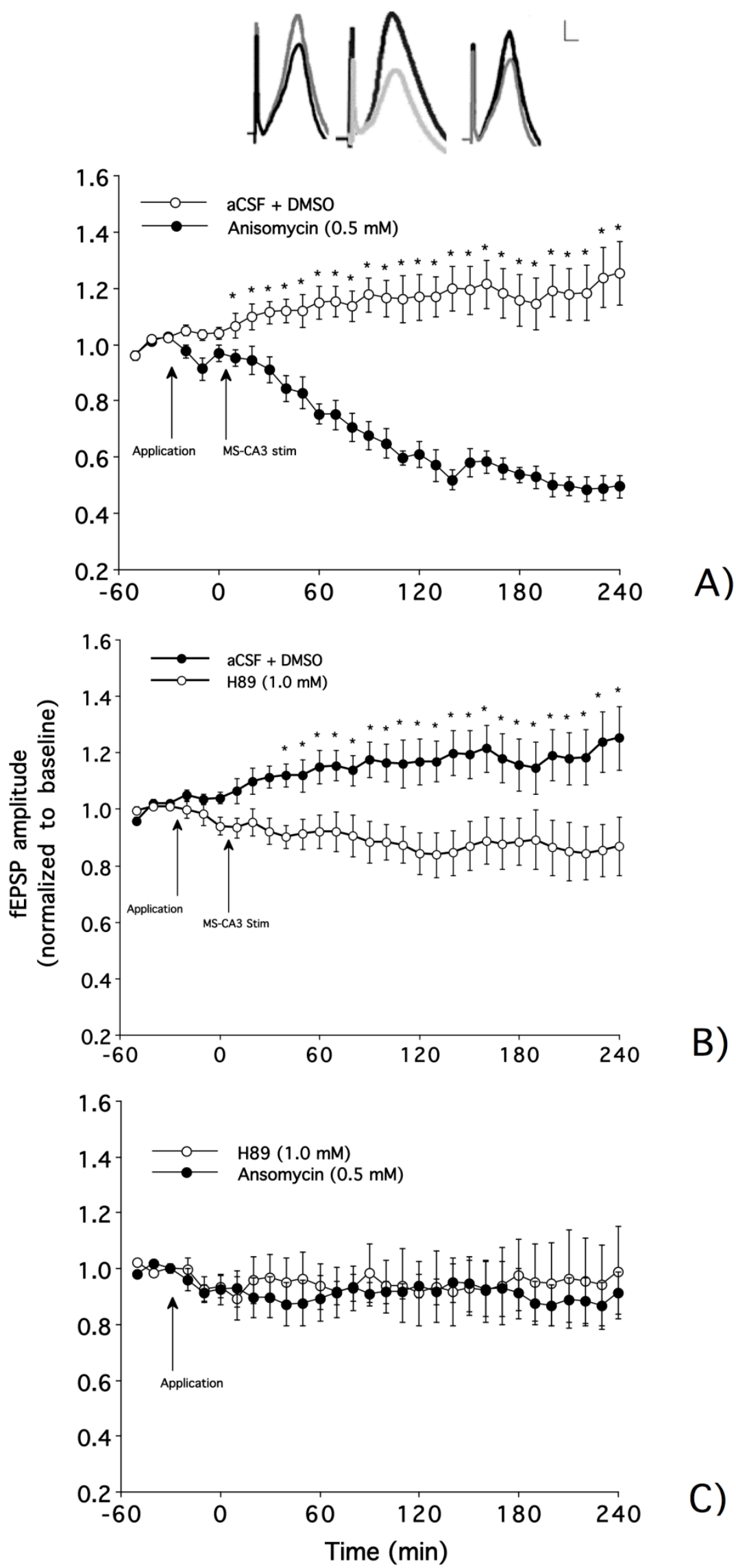
Figure 8. Local application of anisomycin or $\mathbf{H 8 9}$ prevents enhancement of field excitatory postsynaptic potential (fEPSP) amplitude following alternating medial septum (MS)-CA3 stimulation.

In the presence of artificial cerebrospinal fluid (aCSF) and dimethyl sulfoxide (DMSO; $n=8$, applied in CA1 by reverse microdialysis), alternating MS-CA3 stimulation (MS-CA3 Stim., at arrow, same parameters as in Fig. 7) resulted in a gradual increase in fEPSP amplitude over a $4 \mathrm{~h}$ recording period (A and B). In contrast, during local application of anisomycin $(\mathbf{A} ; 0.5 \mathrm{mM}, n=$ 6) or H89 (B; $1 \mathrm{mM}, n=8)$, MS-CA3 stimulation failed to produce this effect. Control experiments (C) assessing changes in fEPSP amplitude during continuous drug application in the absence of delivery of the MS-CA3 protocol revealed that fEPSP amplitude showed a small, but non-significant decline in the presence of anisomycin $(0.5 \mathrm{mM}, n=5)$ and $\mathrm{H} 89(1 \mathrm{mM}, n=4)$. Drugs were applied locally (at arrow) in CA1 after an initial 30 min baseline period recorded in the presence of aCSF + DMSO, and application continued throughout the experiment. Inserts depict fEPSPs during baseline (black) and at the end of the experiment (grey) for an animal receiving aCSF + DMSO (left), anisomycin (middle), or H89 (right). Note the blockade of synaptic potentiation in the rats receiving anisomycin or H89 (fEPSPs are averages of 10 individual sweeps, calibration is $10 \mathrm{~ms}$ and $0.5 \mathrm{mV}$ ). Statistics: ANOVA comparing anisomycin $(0.5 \mathrm{mM})$ and aCSF + DMSO, effect of group, $F_{1,12}=44.95, P<0.0001$, effect of time $F_{29,348}=$ 4.36, $P<0.0001$, group by time interaction, $F_{29,348}=16.62, P<0.0001 ; \mathrm{H} 89$ and aCSF + DMSO, effect of group, $F_{1,14}=8.63, P=0.01$, effect of time $F_{29,406}=0.32, P=0.99$, group by time interaction, $F_{29,406}=3.81, P<0.0001$; anisomycin stimulation vs. anisomycin no stimulation, effect of group, $F_{1,9}=16.95, P=0.01$, effect of time $F_{29,261}=13.75, P<0.0001$, group by time interaction $F_{29,261}=9.59, P<0.0001$; H89 stimulation vs. H89 no stimulation, 
effect of group, $F_{1,10}=0.21, P=0.65$, effect of time $F_{29,290}=1.17, P=0.25$, group by time interaction $F_{29,290}=0,77, P=0.79$. Simple effects of time significant $(P<0.05)$ for all groups receiving MS-CA3 stimulation (panels A and B), but not for groups without stimulation (panel C). * denote significant $(P<0.05$; simple effects tests $)$ group differences. 
amplitude of $85 \%$ of baseline). Application of $\mathrm{H} 89(n=4)$ in the absence of MS-CA3 stimulation did not exert significant effects on fEPSPs amplitude (Fig. 8C; final fEPSP amplitude of 96\% of baseline). The two groups receiving H89 with and without the MS-CA3 protocol did not differ significantly from one another, indicating that LFS was not responsible for the minor depressant effect observed in the MS-CA3 group. Collectively, these experiments demonstrate the importance of activation of PKA and protein synthesis in low frequency-induced MS-H LTP.

\subsubsection{Competition between LTP induced by LFS (1 Hz) and HFS $(100 \mathrm{~Hz})$}

It appears that the mechanisms of MS-H LTP described here and previously (dependence on NMDA receptors, PKA, and protein synthesis) match those involved in classic, HFS-induced LTP at CA1 synapses (Malenka and Nicoll, 1999; Gelinas et al., 2008; Habib and Dringenberg, 2010). Thus, we assessed the relation between MS-H LTP and HFS-induced LTP by occlusion experiments, delivering LFS either before or after HFS stimulation of CA1 afferents. Initially, we asked whether saturation of HFS-LTP interferes with the subsequent induction of LFS-induced MS-H LTP. Following baseline recordings, HFS (100 pulses at $100 \mathrm{~Hz}, n=7)$ was delivered to $\mathrm{CA} 3$, and this protocol was repeated every $10 \mathrm{~min}$ until a saturation point was reached, as indicated by a lack of further potentiation of CA1 fEPSP amplitude after the preceding $100 \mathrm{~Hz}$ train. An average of four (range of 3-6) HFS episodes was required to reach LTP saturation (Fig. 9A; fEPSP amplitude of $213 \%$ of baseline). Subsequent to saturation, the MS-CA3 protocol was delivered (same as above). Surprisingly, under these conditions, this protocol resulted in a gradual, significant decrease of fEPSP amplitude over the remainder of the experiment $(2 \mathrm{~h}$ post LFS; Fig. 9A; final fEPSP amplitude of $171 \%$ of baseline). In contrast, control animals $(n=6)$ that received only CA3 stimulation $(1 \mathrm{~Hz})$ after the initial HFS-induced LTP saturation displayed no significant change in fEPSP amplitude following LFS delivery (Fig. 9A, 209\% of baseline). 
A)

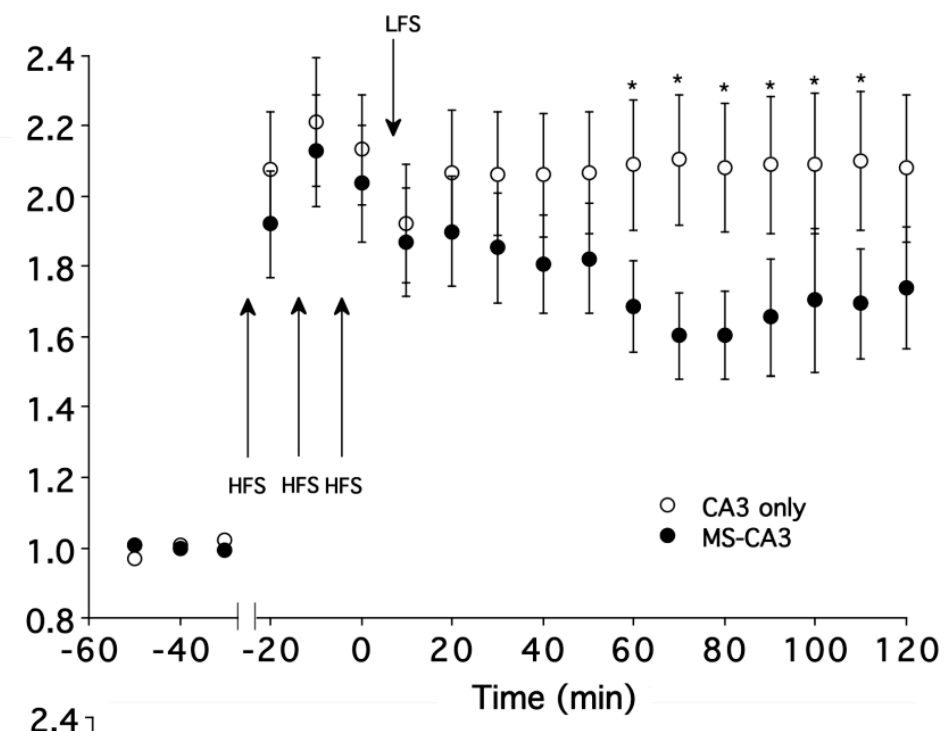

B)
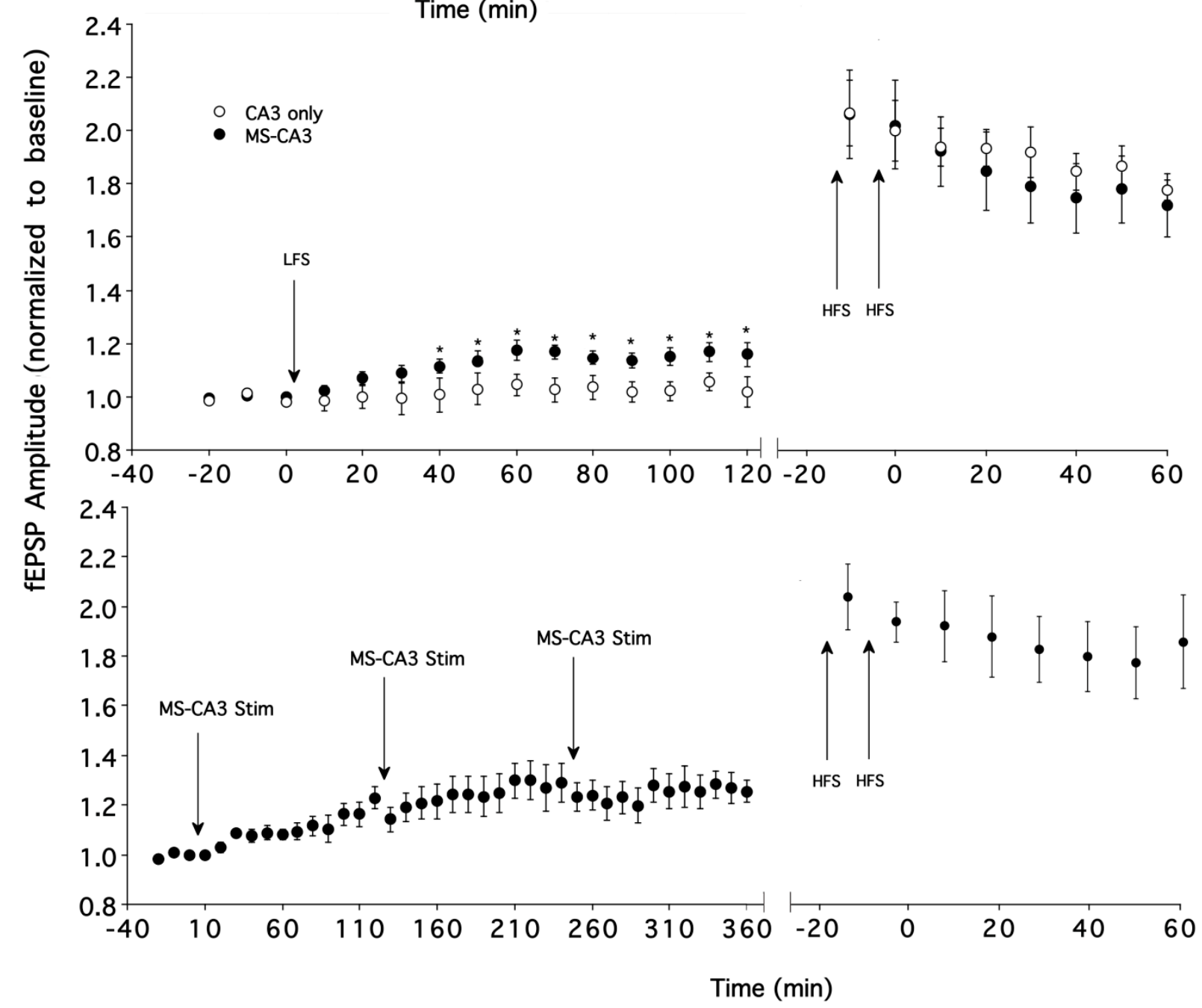
Figure 9. Competition between LTP induced by alternating medial septum (MS)-CA3 stimulation and high frequency stimulation (HFS) of CA3-CA1 synapses
A) Initial HFS (at arrows; 100 pulses at $100 \mathrm{~Hz}$, repeated at 10 min intervals) resulted in saturation of fEPSP amplitude in CA1 (note that separate animals required different numbers of HFS episodes to reach saturation; data shown are the last three 10 min epochs for each rat prior to LFS delivery). Following saturation, animals that received MS-CA3 stimulation (LFS, at arrow) showed a gradual, significant decrease of fEPSP amplitude $(n=7)$, while rats $(n=6)$ receiving CA3 only stimulation (at arrow) did not show a significant amplitude change relative to saturated LTP levels. Statistics: ANOVA comparing MS-CA3 and CA3 only: effect of group $F_{1,11}=1.5, P$ $=0.24$, effect of time, $F_{17,187}=45.6, P<0.0001$; group by time interaction, $F_{17,187}=2.5, P=$ 0.0012. * denote significant $(P<0.05$; simple effects tests $)$ group differences. B) Initial delivery of a single episode of alternating MS-CA3 stimulation (LFS, at arrow) resulted in a gradual, significant amplitude enhancement $(n=6)$, while stimulation of CA3 only showed no significant change in fEPSP amplitude ( $n=6)$. Subsequent delivery of HFS (at arrows; same as above) resulted in identical, maximal (i.e., saturated) levels of LTP in both groups of animals (note that the first data point shown for HFS is the final saturation point for all animals). Statistics: ANOVA comparing MS-CA3 and CA3 only for data prior to HFS, effect of group $F_{1,9}=6.65, P$ $=0.03$, effect of time, $F_{14,126}=5.84, P<0.0001$; group by time interaction, $F_{14,126}=1.94, P=$ 0.03; for data after HFS, effect of group $F_{1,9}=0.12, P=0.74$, effect of time, $F_{7,63}=15.79, P$ $<0.001$; group by time interaction, $F_{7,63}=0.92, P=0.50 . *$ denote significant $(P<0.05$; simple effects tests) group differences. C) Repeated delivery of the MS-CA3 stimulation protocol (at arrows) resulted in a gradual, significant amplitude enhancement over $6 \mathrm{~h}$ that reached saturation after the second LFS episode ( $n=5)$. Subsequent delivery of HFS (at arrows; same as above) 
resulted in similar, saturated levels of LTP as that seen in animals receiving only one episode of MS-CA3 stimulation (see panel 9B). Statistics: ANOVA comparing data obtained after HFS in rats that had received one and repeated episodes of MS-CA3 stimulation, effect of group $F_{1,9}=$ $0.16, P=0.70$, effect of time, $F_{7,63}=13.21, P<0.001 ;$ group by time interaction, $F_{7,63}=0.96, P$ $=0.47)$. 
Consequently, it appears that the effect of MS-CA3 stimulation to induce synaptic potentiation is blocked by the initial saturation of LTP following a standard, HFS protocol.

Subsequently, we carried out experiments using the reverse order of induction protocols, that is, first applying alternating low-frequency MS-CA3 stimulation, which was followed by repeated HFS $(100 \mathrm{~Hz})$ of CA3 until LTP saturation was reached. Consistent with results reported above, an initial, single episode of MS-CA3 stimulation increased fEPSP amplitude over a $2 \mathrm{~h}$ recording period (average fEPSP amplitude of $116 \%$ of baseline during the final 30 min of the experiment). Subsequent delivery of HFS (100 pulses at $100 \mathrm{~Hz}$, every $10 \mathrm{~min}$ ) to CA3 resulted in further fEPSP potentiation, which reached a maximum (i.e., saturation) of $202 \%$ of baseline (Fig 9B). An average of three (range 2-5) HFS bursts was required to reach saturation. In control rats that received initial low frequency CA3 stimulation (100 pulses at $1 \mathrm{~Hz}$ ), fEPSP amplitude remained stable over a $2 \mathrm{~h}$ recording period (Fig. 9B; average fEPSP of 103\%). Subsequent HFS of CA3 resulted in synaptic potentiation to a maximum of $202 \%$ of baseline (Fig. 9B), with an average of five HFS bursts (range 2-8) required to reach saturation.

Moreover, in an additional group of rats, the effects of repeated MS-CA3 stimulation on subsequent HFS-induced LTP were examined. Here, animals received repeated episodes of MSCA3 stimulation at $2 \mathrm{~h}$ intervals until fEPSP amplitude in CA1 could no longer be potentiated using this LFS protocol (3 induction episodes over 6 h; Fig 9C). Amplitude of fEPSPs averaged over 1.5 to $2 \mathrm{~h}$ after each of the first, second, and third induction episodes were $118 \%, 128 \%$, and $127 \%$ of baseline, respectively. Subsequent HFS of CA3 resulted in synaptic potentiation, which saturated at $200 \%$ of baseline (Fig. 9C), with an average of 2 HFS bursts (range 2-3) required to reach this saturation point. 
The fact that final, HFS-induced saturation points were similar in groups that did and did not receive MS-H LTP induction first (between 200\% and 202\%) indicates that LFS and HFS did not result in any detectable, additive effects on synaptic strength. Thus, it appears that MS-CA3 stimulation competes for the same mechanisms also engaged by HFS of afferent fibers to CA1 under the present, experimental conditions.

\subsection{Discussion}

A number of recent papers have demonstrated novel forms of long-lasting synaptic potentiation in amygdalar and hippocampal slice preparations following LFS of various afferent fibers (Mayford et al., 1995; Thomas et al., 1996; Li et al., 2001; Lanté et al., 2006a, b; Huang and Kandel, 2007; Habib and Dringenberg, 2009). Moreover, a LFS-induced, long-lasting form of NMDA receptor-dependent synaptic enhancement has recently been characterized in the CA1 area of the intact hippocampal formation (MS-H LTP; Habib and Dringenberg, 2009). Here, we provide a more comprehensive characterization of cellular mechanisms responsible for MS-H LTP elicited in CA1 of the urethane anesthetized rat preparation. Our experiments demonstrate a critical role of PKA activation and protein synthesis in this novel form of LFS-induced synaptic potentiation. Interestingly, similar mechanisms are known to mediate HFS-induced LTP in CA1 neurons, including NMDA receptor activation, elevation of intracellular $\mathrm{Ca}^{2+}$, activation of multiple kinases such as PKA, and further downstream changes resulting in mRNA transcription and subsequent protein synthesis (Lynch et al., 1983; Izquierdo and Medina, 1995; Lisman et al., 2002; Selcher et al., 2003; Abraham and Williams, 2008; Gelinas et al., 2008). Consistent with this overlap between HFS- and LFS-induced forms of plasticity, we find that initial LTP saturation using $100 \mathrm{~Hz}$ stimulation blocks MS-H LTP, while induction of MS-H LTP competes

with subsequent HFS-LTP. Collectively, these experiments suggest that highly distinct patterns of 
afferent activity (1 vs. $100 \mathrm{~Hz}$ ) can recruit similar mechanisms to elicit long-lasting increases in synaptic strength at CA3-CA1 synapses in vivo.

Initially, we show that alternating, single pulse stimulation of the MS and CA3 area (50 alternating stimulation pulses each, $1 \mathrm{~s} \mathrm{ISI}$ ) elicits synaptic potentiation of fEPSPs recorded in the contralateral CA1 area. In contrast, CA3 stimulation alone (100 pulses, $1 \mathrm{~Hz})$ did not result in significant changes in fEPSP amplitude. Similarly, previous work has shown that stimulation of the MS only (i.e., in the absence of CA3 pulses) also does not elicit fEPSP potentiation, observations that demonstrate the critical requirement for co-activation of MS and CA3 fibers for the induction of MS-H LTP (Habib and Dringenberg, 2009).

As mentioned above, the mechanisms of MS-H LTP described here exhibit surprising parallels to those involved in HFS-induced LTP in the CA1 field. Several, other forms of LFSinduced potentiation known to occur in the amygdala and hippocampus also appear to involve similar signalling mechanisms. Low frequency induced synaptic potentiation in CA1 pyramidal and basolateral amygdala neurons in vitro is blocked in the presence of PKA or protein synthesis inhibitors, confirming the critical role of these signalling pathways in both slice and intact preparations (Lanté et al., 2006b; Huang and Kandel, 2007). At present, the only, clear difference among various types of LFS-induced synaptic potentiation lies in the requirement for NMDA receptor activation in MS-H LTP (Habib and Dringenberg, 2009). All other forms of LFSinduced enhancement can readily be elicited under conditions of NMDA receptor blockade and appear to be mediated by either mGluR5 (in CA1; Lanté et al., 2006b), kainate-type ionotropic glutamate receptors (in lateral amygdala; Li et al., 2001), or co-activation of several neuromodulatory binding sites (5-HT4, dopamine D1, and 3 -adrenergic receptors in the basolateral amygdala; Huang and Kandel, 2007). 
To further assess the putative overlap between cellular mechanisms involved in MS-H LTP and HFS-induced LTP, we carried out additional experiments to examine potential competition and/or occlusion between these LTP induced by these distinct (1 vs. $100 \mathrm{~Hz})$ induction protocols. Initial HFS to saturate LTP blocked further MS-H LTP and, in fact, reversed the effect of alternating MS-CA3 stimulation from potentiation to synaptic depression. Similarly, initial induction of MS-H LTP reduced the amount of subsequent HFS-LTP, so that final, saturated levels of LTP were the same in rats that did not receive initial MS-CA3 stimulation. The complete absence of any additive or synergistic effects of HFS and LFS delivered to the same animal provides an important, further confirmation of the mechanistic similarity of synaptic potentiation elicited by these different stimulation protocols under the present, experimental conditions.

The experimental evidence presented here raises the intriguing and puzzling question as to how such distinct stimulation patterns can recruit apparently identical receptor and intracellular mechanisms. Typically, LFS (usually $1 \mathrm{~Hz}$ ) results in a moderate increase in intracellular $\mathrm{Ca}^{2+}$ levels, leading to a depression of synaptic efficacy, while greater influx of $\mathrm{Ca}^{2+}$ (as seen with various HFS protocols) elicits LTP (Teyler et al., 1994; Debanne, 1996; see also Massey and Bashir, 2007). The observation that alternating $(1 \mathrm{~Hz})$ stimulation of septal and commissural fibers resulted in synaptic enhancement suggests that intracellular $\mathrm{Ca}^{2+}$ levels were higher than those seen with standard $(1 \mathrm{~Hz})$ LTD induction protocols, even though they clearly must be below levels seen with HFS, which results in much greater levels of potentiation that those seen in MSH LTP. Certainly, it appears that low-frequency, co-activation of converging afferents (also see Popescu et al., 2007) to the CA1 area constitutes a more effective means of NMDA receptor activation $/ \mathrm{Ca}^{2}$ influx than protocols consisting of stimulation of only one set of afferent fibers 
(Dudek and Bear, 1992). At present, direct support for these assumptions is lacking, but the use of $\mathrm{Ca}^{2+}$ imaging techniques and direct recordings of NMDA receptor currents might allow for their assessment.

An important, unanswered question concerns the functional, behavioral significance of MS-H LTP characterized here and previously. Recent, preliminary experiments have shown that spatial (water maze) acquisition, a type of learning critically dependent on the integrity of the septohippocampal system (Frielingsdorf et al., 2006; Dashniani et al., 2009), results in the occlusion of MS-H LTP at $3 \mathrm{~h}$ but not $24 \mathrm{~h}$ after training (D. Habib and H.C. Dringenberg, unpublished observations). Similar occlusion/saturation approaches (Martin and Morris, 2002) have been used to confirm the role of LTP in hippocampal (e.g., inhibitory avoidance; Whitlock et al., 2006) and neocortex-dependent learning (e.g., motor learning, Rioult-Pedotti et al., 1998). If confirmed, these data would support a possible role of MS-H LTP in early stages of consolidation of spatial information encoding in hippocampal circuits.

In summary, the present set of experiments demonstrates the critical role of PKA and protein synthesis as mediators of a novel form of plasticity induced by LFS of MS and CA3 afferents to the CA1 area in vivo. Further, MS-H LTP is blocked by preceding saturation of HFSinduced LTP and there are no additive effects when initial MS-H LTP is followed by HFS-LTP, data that further confirm the similarity of receptor and intracellular signals mediating these two forms of plasticity. Intriguing questions arising from this work concern the mechanisms of effective NMDA receptor activation by low frequency inputs, as well as the integrative functions served by LFS-induced synaptic potentiation. Answers to these issues will provide important information regarding the cellular mechanisms and functional significance of low frequencyinduced synaptic enhancement in the mammalian brain. 


\section{Chapter 5. Influence of age and behavioural experience on the expression of MS-H LTP}

\subsection{Abstract}

The relation between long-term potentiation (LTP) and learning was, in part, established by earlier studies demonstrating a relation between memory loss and decay of LTP in aging, as well as more recent work on LTP-like synaptic enhancement following behavioral experiences. The majority of experimental work to date has used high frequency stimulation (HFS) protocols as a model to mimic synaptic potentiation thought to occur during memory formation after behavioral training. Interestingly, is it now well established that low frequency stimulation (LFS) patterns are also effective in the induction of LTP-like plasticity in the hippocampal formation and amygdala. For example, a series of previous reports have shown that alternating LFS $(1 \mathrm{~Hz})$ to the medial septum (MS) and CA3 hippocampal area (MS-CA3 stimulation) elicits a NMDA receptordependent long-lasting synaptic enhancement (MS-H LTP) in CA1 pyramidal cells. Here, the first set of experiments demonstrated that similar to LTP induced by HFS, low frequency-induced MS-H LTP is compromised in early aging, while similar levels of potentiation are expressed in the juvenile and adult hippocampus in vivo. In a separate set of experiments, the influence of water maze training on the subsequent expression of MS-H LTP was examined in adult rats. These data showed that alternating MS-CA3 stimulation $3 \mathrm{~h}$ after training on the hidden platform version of the Morris water maze task failed to elicit MS-H LTP, an occlusion that was not observed at 8 and $24 \mathrm{~h}$ after training. Further, this occlusion was not seen in yoked swim controls or animals trained on the visible platform version of the task. Collectively, these experiments are 
the first to characterize age-related changes in LFS-LTP in vivo and provide compelling evidence that MS-H LTP is involved in early stages of consolidation of spatial memory in local hippocampal circuits. 


\subsection{Introduction}

Modifications in synaptic strength such as long-term potentiation (LTP) are generally assumed to be the mechanisms by which memory traces are initially encoded, consolidated and stored in the central nervous system (Bliss and Lómo, 1973; Martin et al., 2000). Experimentally, LTP can be effectively induced by applying high-frequency stimulation (HFS; tetanic) to afferent fibers, resulting in long-lasting synaptic enhancement (Bliss and Lómo, 1973; Albensi et al., 2007; Caporale and Dan, 2008; Raymond, 2007). This experimental induction of HFS-induced LTP displays physiological properties that are known to govern learning and memory at the behavioural level (McNaughton, 1983; Bliss and Collingridge, 1993). Some of the initial evidence that established a relation between LTP and learning and memory functions came from correlational studies in the aged hippocampus (Eichenbaum, 1996; Hölscher, 1999; Martin et al., 2000). For example, aged rats often show deficits in the acquisition and/or retention of spatial memories, as well as in the induction and maintenance of hippocampal LTP induced by robust HFS protocols (e.g., 100-200 Hz). In fact, the decline in retention performance in aged rats has been found to correlate with an accelerated decay of LTP in aged rodents (Barnes, 1979; Tielen et al., 1983; Barnes and McNaughton, 1985; Rosenzweig et al., 1997; Bach et al., 1999). These data demonstrate a close relation between the ability to perform hippocampal-dependent memory tasks and deficits in synaptic potentiation.

Remarkably, there is now considerable evidence demonstrating an even more direct relation between learning (i.e., training) and plasticity in the neocortex and various subcortical areas (Kirkwood et al., 1996; Rogan et al., 1997; Rioult-Pedotti et al., 2000; Whitlock et al., 
2006; Hager and Dringenberg, in press). For example, rats trained on a complex reaching task involving only one of the two forelimbs (with the other limb serving as control) showed an enhancement in field potential amplitude in the primary motor cortex (M1), an effect seen only in the cortical hemisphere contralateral to a trained forelimb. Importantly, this facilitation of synaptic strength following training limited the subsequent induction of LTP induced by high frequency electrical stimulation (Rioult-Pedotti et al., 2000). Similarly, in freely moving rats, fear conditioning enhances field potentials in the lateral amygdala to levels comparable to those induced by HFS without training (Rogan et al., 1995; 1997). More recent findings reported an enhancement in field excitatory postsynaptic potential (fEPSP) amplitude in the CA1 area of the hippocampus in rats after inhibitory avoidance learning, a form of synaptic facilitation that occluded LTP subsequently induced by HFS patterns (Whitlock et al., 2006). Together, these data suggest that learning and memory-related functions result in an up-regulation of synaptic efficacy, mechanisms that appear to be similar to those engaged during the induction of HFSelicited LTP at cortical and sub-cortical synapses

As summarized above, the relation between LTP and learning was, in part, established by earlier, correlational studies of behaviour and LTP in aging, as well as more recent work on LTPlike synaptic enhancement following behavioral experiences. It is noteworthy to mention, however, that the experimental work to date has used only HFS protocols as a model to mimic synaptic potentiation thought to occur during memory formation after behavioral training. Interestingly, several recent reports have now confirmed that low frequency stimulation (LFS) patterns are also effective in the induction of long-lasting synaptic facilitation in the amygdala and hippocampal formation (Mayford et al., 1995; Thomas et al., 1996; Li et al., 1998; Lanté et al., 2006a, b; Huang and Kandel, 2007; Habib and Dringenberg, 2009, in press). For example, in 
urethane-anesthetised rats, fEPSPs in area CA1 of the hippocampus show significant, long lasting potentiation $(>5 \mathrm{~h})$ following alternating, single pulse stimulation (at $1 \mathrm{~Hz})$ of the medial septum (MS) and hippocampal (H) CA3 area (MS-H LTP; Habib and Dringenberg, 2009). Similar to most types of classical HFS-LTP, MS-H LTP requires NMDA receptor activation, protein synthesis and protein kinase A activity (Habib and Dringenberg, in press). Evidently, certain forms of LFS-LTP demonstrate mechanistic similarities to those mediating classical HFS-LTP. However, whether this type of synaptic potentiation shows age-related changes similar to those occurring for HFS-LTP is unknown. Similarly, the behavioural significance of MS-H LTP has never been investigated.

Here, in separate experiments, we address these two unanswered questions: First, what is the influence of age on the expression of MS-H LTP (Experiment 1)? These series of experiments showed that similar to LTP induced by HFS, low frequency-induced MS-H LTP is compromised in early aging, while similar levels of potentiation are expressed in the juvenile and adult hippocampus. These findings are the first to suggest that forms of LTP induced by highly distinctive low vs. high frequency patterns are equally compromised in the early aged brain. Next, we addressed the important question regarding the functional significance of MS-H LTP (Experiment 2). In these experiments, LFS was delivered exactly at 3, 8, or $24 \mathrm{~h}$ after the end of behavioural (water maze) training and results showed that MS-H LTP could not be induced (i.e., was occluded) $3 \mathrm{~h}$ after training, while it was intact at 8 and $24 \mathrm{~h}$ intervals. Further, this occlusion was not seen in swim controls or animals trained on the visible platform version of the task. Collectively, these experiments are the first to characterize age-related changes in LFS-LTP in vivo and provide compelling evidence that MS-H LTP is involved in early stages of consolidation of hippocampal-dependent learning tasks. 


\subsection{Materials and Methods}

\subsubsection{Subjects}

For Experiment 1 (age comparison), procedures were performed on 27-33 day (juvenile; 75-130g), 3-4 month (adult; 310-500g) and 14 month-old (early-aged; 800-1000g) male LongEvans rats. For Experiment 2 (water maze training), procedures were conducted in adult (310-450 g) rats. All animals were housed in a colony room (12/12-h reverse light cycle) with food and water available ad libitum. The procedures were conducted in accordance with the guidelines of the Canadian Council on Animal Care and approved by the Queen's University Animal Care Committee. Each rat was used for only one experiment.

\subsubsection{Experiment 1: Influence of age on expression of MS-H LTP}

\subsubsection{Surgical preparation}

All electrophysiological experiments were conducted under deep urethane anesthesia (1.5 $\mathrm{g} / \mathrm{kg}$, intraperitoneal [i.p.], administered in three $0.5 \mathrm{~g} / \mathrm{kg}$ doses every $20 \mathrm{~min}$ and supplemented with $0.2-0.6 \mathrm{~g} / \mathrm{kg}$ as required prior to the onset of data collection). Additionally, the local anesthetic Marcaine $(5 \mathrm{mg} / \mathrm{ml})$ was applied to the skin overlying the skull along the incision line (3-4 subcutaneous injections, approximately $0.03 \mathrm{ml}$ each) $15 \mathrm{~min}$ prior to incision.

Rats of the different ages specified above were placed in a stereotaxic apparatus and body temperature was maintained between 36 and $37^{\circ} \mathrm{C}$ by means of an electrical heating blanket. An incision was made to expose the skull surface and small skull holes were drilled above the CA3 region (AP -4.16, ML +3.0, $\mathrm{V}-4.0$ ), the contralateral CA1 area (AP -4.16, ML -3.0 V -3.0), and at bregma point (AP 0.0, ML 0.0, V - 6.5) to gain access to the MS. Note that, for 14 month-old rats, the medial-lateral coordinates of skull holes above CA1 and CA3 were adjusted slightly 
(CA1: ML -3.1; CA3: ML +3.1). Skull holes for ground and reference electrodes (jewellery screws attached to miniature connectors) were made in the bone overlying the prefrontal cortex and cerebellum, respectively. An additional skull hole for a septal stimulation return electrode (jewellery screw) was drilled adjacent to bregma. All stereotaxic measurements were based on the anatomical work of Paxinos and Watson (1998). Final ventral placements of the CA3 stimulation and the CA1 recording electrode were adjusted to elicit maximal amplitude field excitatory postsynaptic potentials (fEPSPs) and paired-pulse facilitation (100 ms interstimulus interval, ISI) in area CA1 in response to contralateral CA3 stimulation.

\subsubsection{Electrophysiology and Experimental Procedures}

Stimulation of CA3 $(0.2 \mathrm{~ms}$ pulses every $30 \mathrm{~s}$, intensity adjusted to yield $50-60 \%$ of maximal fEPSP amplitude) was provided by a concentric bipolar electrode (Rhodes Medical Instruments Series 100, David Kopf, Tujunga, CA, USA) connected to a stimulus isolation unit providing a constant current output (PowerLab/16 s system with ML 180 Stimulus Isolator, AD Instruments, Toronto, Canada). MS stimulation was provided through a monopolar electrode (Teflon insulated stainless steel, 125 - $\mu \mathrm{m}$ tip diameter) delivering single, negative constant-current pulses (0.2 ms duration, $0.5 \mathrm{~mA})$.

All fEPSPs were differentially recorded (Teflon insulated stainless steel wire, $125-\mu \mathrm{m}$ tip diameter) against a reference electrode screw placed in the bone overlaying the cerebellum. The CA1 signals were amplified, filtered $(0.3-1 \mathrm{kHz})$, digitized $(10 \mathrm{kHz})$, and stored for subsequent off-line analysis using the PowerLab system running Scope software (v. 4.0.2).

Prior to formal data collection, input-output curves were established for each animal by recording fEPSPs in CA1 in response to CA3 stimulation between 0.1 and $1.0 \mathrm{~mA}(0.1 \mathrm{~mA}$ increments). Based on these input-output curves, a stimulation intensity eliciting between $50-60 \%$ 
of the maximal fEPSP amplitude was chosen for the subsequent experiment. For each rat, 60 initial baseline fEPSPs (every $30 \mathrm{~s}$ ) were recorded. Subsequently, the MS-CA3 alternating stimulation protocol was delivered, consisting of 50 single pulses to both the MS and CA3 area, delivered at $0.5 \mathrm{~Hz}$, with the ISI of $1000 \mathrm{~ms}$ between successive MS and CA3 pulses). For all experiments, the MS-CA3 alternating stimulation was delivered twice, with the second delivery occurring $2 \mathrm{~h}$ after the initial MS-CA3 stimulation. Recordings of fEPSPs in CA1 (every $30 \mathrm{~s}$, as in baseline) in response to single pulse CA3 stimulation continued for $2 \mathrm{~h}$ after the delivery of each episode of the MS-CA3 protocol. Further, to verify that alternating co-activation of both MS and CA3 fibers is required to elicit LFS-induced potentiation, control animals that received only CA3 pulses during the induction protocol $(100$ pulses at $1 \mathrm{~Hz})$ were studied in all three age groups.

At the end of each experiment, rats were perfused through the heart with $10 \%$ formalin, their brains were extracted, and standard histological techniques were used to verify all electrode placements. Data obtained with inaccurate placements were excluded from the data analysis.

\subsubsection{Data Analysis}

All data are expressed as a mean \pm standard error of mean (S.E.M). The maximal fEPSP amplitude was analyzed offline by the Scope software. Subsequently, amplitude data were averaged over 10-min intervals, and these averages were normalized by dividing all data for each rat by the average baseline (pre-MS-CA3 alternating stimulation) amplitude of that animal. All data were analyzed using analyses of variance (ANOVA) and, where statistically appropriate, simple effects tests, all of which were computed using the software package CLR ANOVA (v.1.1, Clear Lake Research Inc., Houston, TX). A Tukey's post hoc analysis was used for individual comparisons of fEPSP amplitude in juvenile, adult and early-aged animals. 


\subsubsection{Experiment 2: Influence of behavioral experience (water maze training) on expression of MS-H LTP}

\subsubsection{Behavioral training}

The water maze consisted of a circular pool (1.8-m diameter) filled with water $\left(23-25^{\circ} \mathrm{C}\right)$ made opaque by adding nontoxic white paint. A circular escape platform $(30 \mathrm{~cm}$ in diameter) was submerged about $2 \mathrm{~cm}$ below the surface of the water, located away from the pool wall in the centre of one of the four pool quadrants.

Rats were trained by administering 12 training trials, divided into three blocks of four trials each, with a 5 min rest period between blocks. For each trial block, four different start positions from the four cardinal compass points were used in random order. A trial started with the animal being released into the pool facing the pool wall and was then allowed to swim freely, for a maximum of $60 \mathrm{~s}$, to locate the hidden escape platform. After successfully mounting the platform, the animal was allowed to remain on the platform for $15 \mathrm{~s}$ before commencement of the next trial. Animals that failed to locate the platform within $60 \mathrm{~s}$ were guided by hand and then placed on the platform for $15 \mathrm{~s}$ before commencement of the next trial. The submerged platform was kept in the same location for all 12 trials. The time to reach the escape platform was recorded for each trial by the experimenter using a digital stopwatch. An additional group of rats was trained on the visible platform version of the water maze (same training procedure as above, unless otherwise noted) using the same platform ( $2 \mathrm{~cm}$ below the water surface), the location of which was indicated by a clearly visible ensign [clear plastic cylinder $(\sim 10 \mathrm{~cm}$ in height $)$ with black stripes] placed on top of the platform. For visible platform training, the platform and ensign were randomly moved to a new pool quadrant for each trial, with the restriction that the same quadrant was used only once for each trial block. In a further group of yoked control animals, a rat was matched to each rat in the hidden platform group and swam for the same duration as that 
rat, but without a platform present in the pool. After the end of water maze training, each rat was placed under a heat lamp for about 20 min prior to being returned to the colony room.

\subsubsection{Electrophysiology}

Electrophysiological procedures were carried out so that the delivery of the MS-CA3 stimulation protocol occurred exactly at 3,8 , or $24 \mathrm{~h}$ after the end of behavioural training. Further, the first injection of urethane was given about $2 \mathrm{~h}$ prior to the time of delivery of the MSCA3 protocol.

The same electrophysiological procedures were used as described above for Experiment 1, with the following exceptions: (a) only one MS-CA3 stimulation protocol was delivered to each animal, with recording continuing for 2 hours following protocol delivery.

\subsubsection{Data Analysis}

All water maze and electrophysiological data was analyzed using analyses of variance (ANOVA) and, where statistically appropriate, simple effects tests were computed using the software package CLR ANOVA (v.1.1, Clear Lake Research Inc., Houston, TX).

\subsection{Results}

\subsubsection{Experiment 1}

5.4.1.1 MS-H LTP in the juvenile (27-33 days), adult (3-4 months) and early aged (14 months) hippocampus

In urethane anesthetised rats, single stimulation pulses applied to area CA3 produced positive-going fEPSPs (latency to peak about 10-11 ms) recorded in the stratum radiatum of the contralateral CA1 area in juvenile, adult and early aged rats (Fig. 10 insert). Delivery of the stimulation protocol consisting of alternating single pulses applied to the MS and CA3 produced a 
gradual, significant increase in fEPSP amplitude over the course of the experiment in juvenile ( $n$ $=6)$ and adult $(n=7)$ rats. (Fig. 10A; MS-CA3 stimulation consisted of a total of 50 pulses at 0.5 $\mathrm{Hz}$ for each area, starting with MS, $1000 \mathrm{~ms}$ ISI). In juvenile rats, amplitude of fEPSPs averaged over the 1.5 to $2 \mathrm{~h}$ epochs after delivery of the first and second induction episode were 117 and $122 \%$ of baseline, respectively (Fig. 10A). Similarly, delivery of the MS-CA3 stimulation in adult rats resulted in fEPSP amplitude values of 120 and 135\% of baseline after one and two induction episodes, respectively (values are averages over 30 min of recording between 1.5-2 h after protocol delivery; Fig. 10A). Conversely, the MS-CA3 stimulation protocol failed to elicit enhancement in fEPSP amplitude in early aged rats $(n=7)$, with an average fEPSP amplitude of 96 and $80 \%$ of baseline during the last $1.5-2 \mathrm{~h}$ epoch after one and two induction episodes, respectively.

Next, we asked whether the change in fEPSP amplitude seen in all three age groups requires co-activation of the MS and CA3, or whether similar effects are seen with CA3 stimulation only (Fig 10B-D; 100 single pulses to CA3 at $1 \mathrm{~Hz}$ for each induction episode). For both juveniles ( $n=5$, Fig. 10B) and adults ( $n=5$; Fig. 10C), no enhancement of fEPSP amplitude was apparent with only CA3 stimulation, with a mean amplitude of 100 and $92 \%$ of baseline in juvenile and adult rats, respectively, during the last $30 \mathrm{~min}$ of the experiment. Thus, it appears that co-activation of medial septal and hippocampal fibers is required for the effects described above. In early aged rats $(n=4$; Fig. $10 \mathrm{D})$ receiving only CA3 stimulation, fEPSP amplitude in area CA1 did not show significant changes during the course of the experiment, with fEPSP amplitude at $89 \%$ of baseline during the final 30 min of the experiment. 


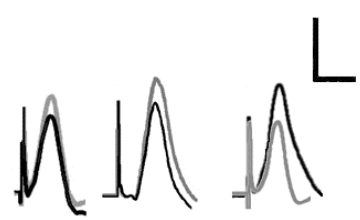

A. Age comparison
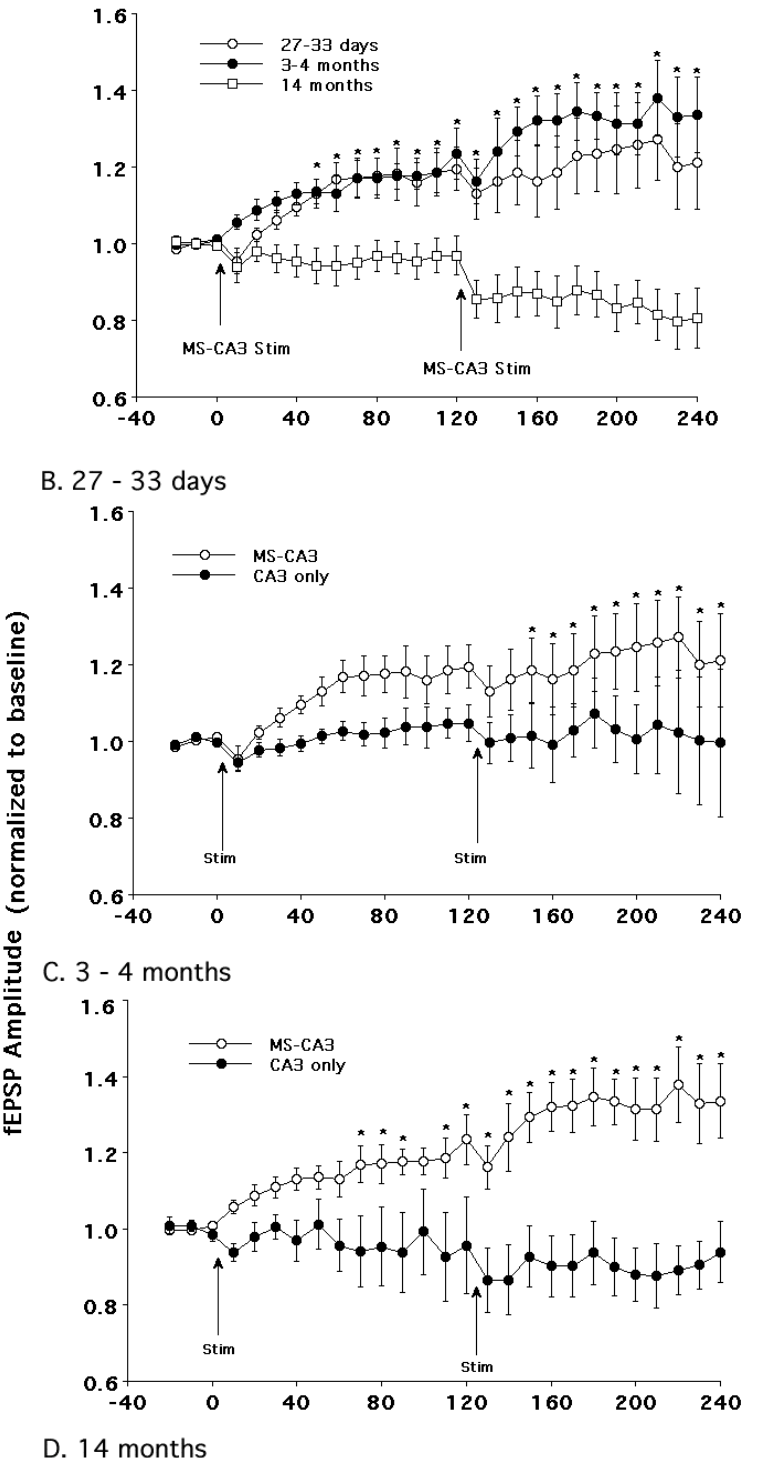

D. 14 months

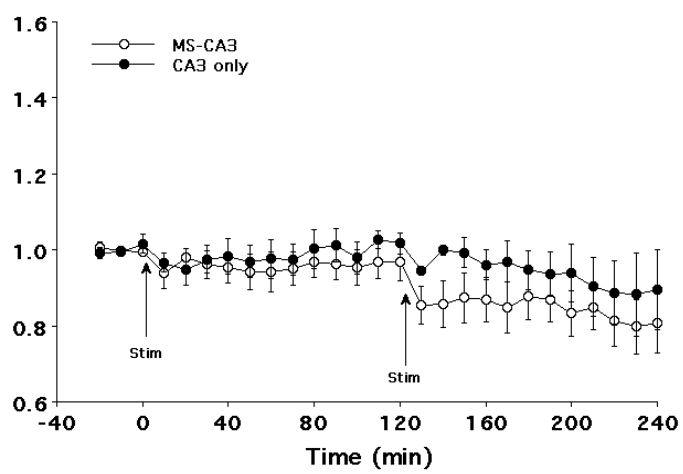


Figure 10. The effects of alternating medial septum (MS)-CA3 stimulation on amplitude of CA1 field excitatory postsynaptic potentials (fEPSPs) in 27-33 day (juvenile), 3-4 month (adult) and 14-month-old (early aged) rats.

A) Delivery of two episodes (2 $\mathrm{h}$ apart, at arrows) of the alternating MS-CA3 stimulation protocol (50 pulses to each MS and CA3, $1000 \mathrm{~ms}$ inter-stimulus interval) resulted in a gradual enhancement of fEPSP amplitude in juveniles $(n=6)$ and adults $(n=7)$ but not in early aged rats $(n$ $=7$; B-D) Comparisons of the effect of the MS-CA3 protocol to stimulation of only CA3 (at arrow; 100 CA3 pulses, $1 \mathrm{~Hz}$ ) for each age group $(\mathbf{B})$ juvenile, $\mathrm{n}=5$; C) adult, $\mathrm{n}=5$; D) early aged, $n=4$. Delivery of CA3 only stimulation failed to induce synaptic enhancement in all age groups. Inserts depict fEPSPs during baseline (black) and at the end of the experiment (grey) for a juvenile (left), adult (middle), and early aged (right) animal receiving MS-CA3 stimulation (fEPSPs are averages of 10 individual sweeps, calibration is $10 \mathrm{~ms}$ and $1.0 \mathrm{mV}$ ). Statistics : A) ANOVA comparing MS-CA3 stimulation in three age groups; effect of group $\mathrm{F}_{2,17}=10.8, P=$ 0.818 , effect of time, $\mathrm{F}_{26,442}=4.7, P<0.001$, group by time interaction, $\mathrm{F}_{52,442}=6.1, P<0.001$. Tukey's post hoc analysis showed 14-month rats differed significantly from both other age conditions $(P=0.008)$. B) ANOVA comparing MS-CA3 stimulation and CA3 only; juvenile rats, effect of group $\mathrm{F}_{1,10}=3.8, P=0.078$, effect of time, $\mathrm{F}_{26,260}=2.0, P=0.003$, group by time interaction, $\mathrm{F}_{26,260}=1.6, P=0.025 ; \mathrm{C}$ ) adult rats, effect of group $\mathrm{F}_{1,10}=13.5, P=0.004$, effect of time, $\mathrm{F}_{26,260}=1.8, P=0.010$, group by time interaction, $\left.\mathrm{F}_{26,260}=5.2, P<0.0001 ; \mathrm{D}\right)$ early aged rats, effect of group $\mathrm{F}_{1,9}=0.71, P=0.419$, effect of time, $\mathrm{F}_{26,234}=4.1, P<0.0001$, group by time interaction, $\mathrm{F}_{26,234}=0.7, P=0.77$. Simple effects of time significant for animals receiving MS-CA3 stimulation (juvenile, $\mathrm{F}_{26,234}=4.1, P<0.0001$; adult, $\mathrm{F}_{26,156}=7.7, P<0.001$; early 
aged; $\mathrm{F}_{26,156}=4.4, P<0.001$ ) but not for animals receiving $\mathrm{CA} 3$ only stimulation (juvenile, $\mathrm{F}$ $26,104=0.2, P=1.000 ;$ adult, $\mathrm{F}_{26,104}=0.7, P=0.818$; early aged; $\left.\mathrm{F}_{26,78}=1.3, P=0.139\right) .{ }^{*}$ denote significant $(P<0.05 ;$ simple effect tests $)$ group differences. 


\subsubsection{Experiment 2}

\subsubsection{Effects of water maze training on MS-H LTP}

\subsection{Behavioural Training}

The average escape latency for all rats trained on the hidden $(n=20)$ and visible $(n=7)$ platform version of the Morris water maze (MWM) is shown in Fig. 11. Both groups of rats showed significant decreases in escape latency over 12 trials, with animals trained on the visible platform showing overall faster escape latencies relative to rats trained on the hidden platform version of the task (Fig. 11).

\subsection{Electrophysiology}

Subsequent electrophysiological procedures were carried out so that the delivery of the MS-CA3 stimulation protocol occurred exactly at 3, 8, or $24 \mathrm{~h}$ after the end of behavioral training (Fig. 12). Alternating MS-CA3 stimulation delivered at $8 \mathrm{~h}(n=6)$ or $24 \mathrm{~h}(n=7)$ after the end of training on the hidden platform task resulted in an enhancement of fEPSP amplitude in CA1 to 119 and $117 \%$ of baseline, respectively (Fig. 12A; fEPSP amplitude averaged over last 30 min of the experiment). These values in enhancement are equivalent to those reported previously (see chapters 2 and 4) for delivery of one MS-CA3 stimulation protocol. Remarkably, however, rats that received the protocol $3 \mathrm{~h}$ after training failed to show the typical enhancement in fEPSP amplitude normally seen with MS-CA3 stimulation (average fEPSP amplitude $98 \%$ of baseline during last half hour of the experiment; Fig. 12A). 


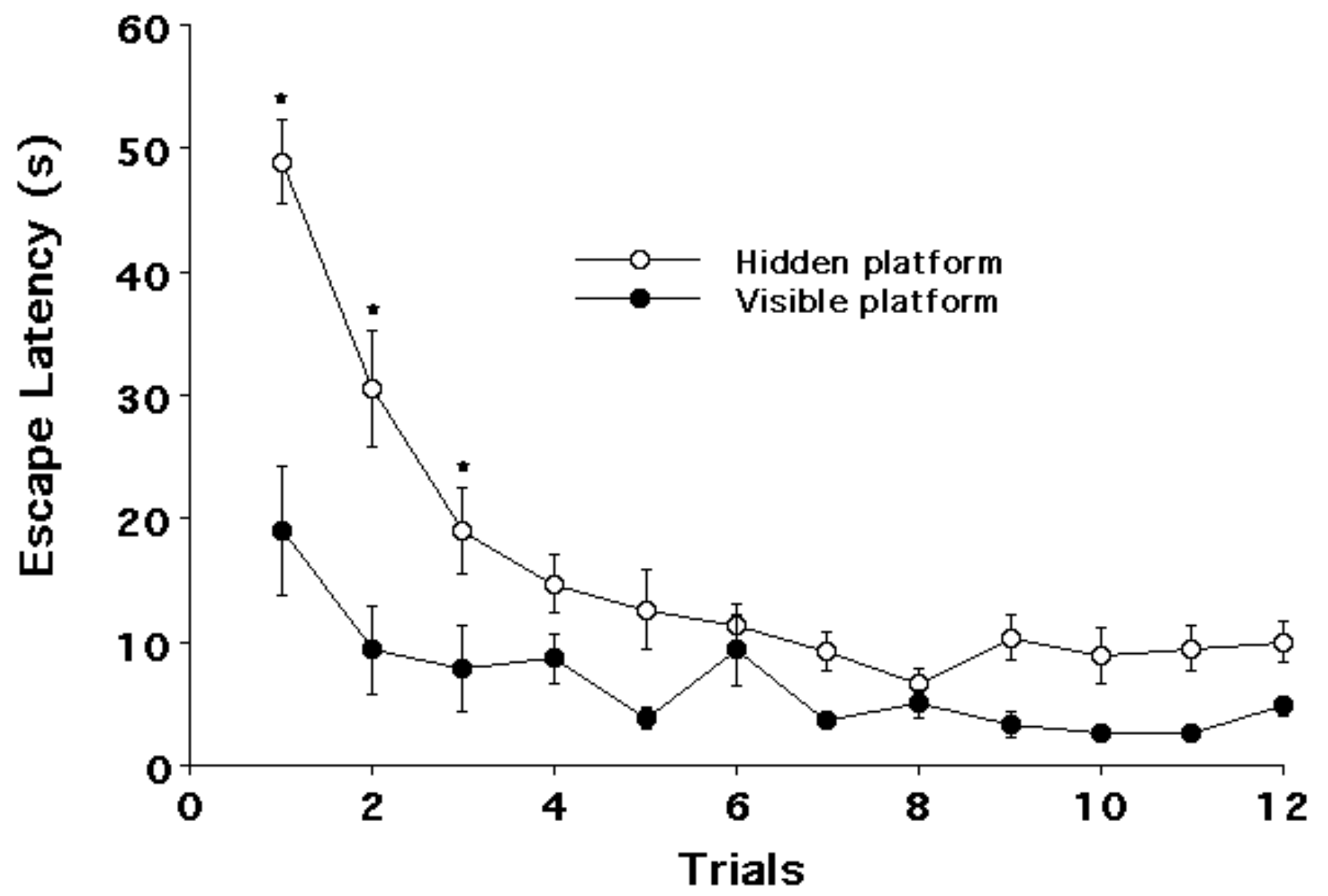

Figure 11. Average escape latency across 12 trails for rats trained on the hidden $(n=20)$ and visible platform $(n=7)$ version of the Morris water maze task.

Statistics: ANOVA: hidden vs. visible platform, effect of group $\mathrm{F}_{1,25}=16.7, P=0.0004$, effect of time, $\mathrm{F}_{11,275}=15.1, P<0.0001$, group by time interaction, $\mathrm{F}_{11,275}=2.3, P=0.008$. Simple effects of time significant for rats' escape latencies in hidden $\left(\mathrm{F}_{11,209}=23.2, P<0.0001\right)$ and visible platform $\left(\mathrm{F}_{11,66}=5.5, P<0.0001\right)$ version of the task. $*$ denote significant $(P<0.05$; simple effect tests) group differences. 


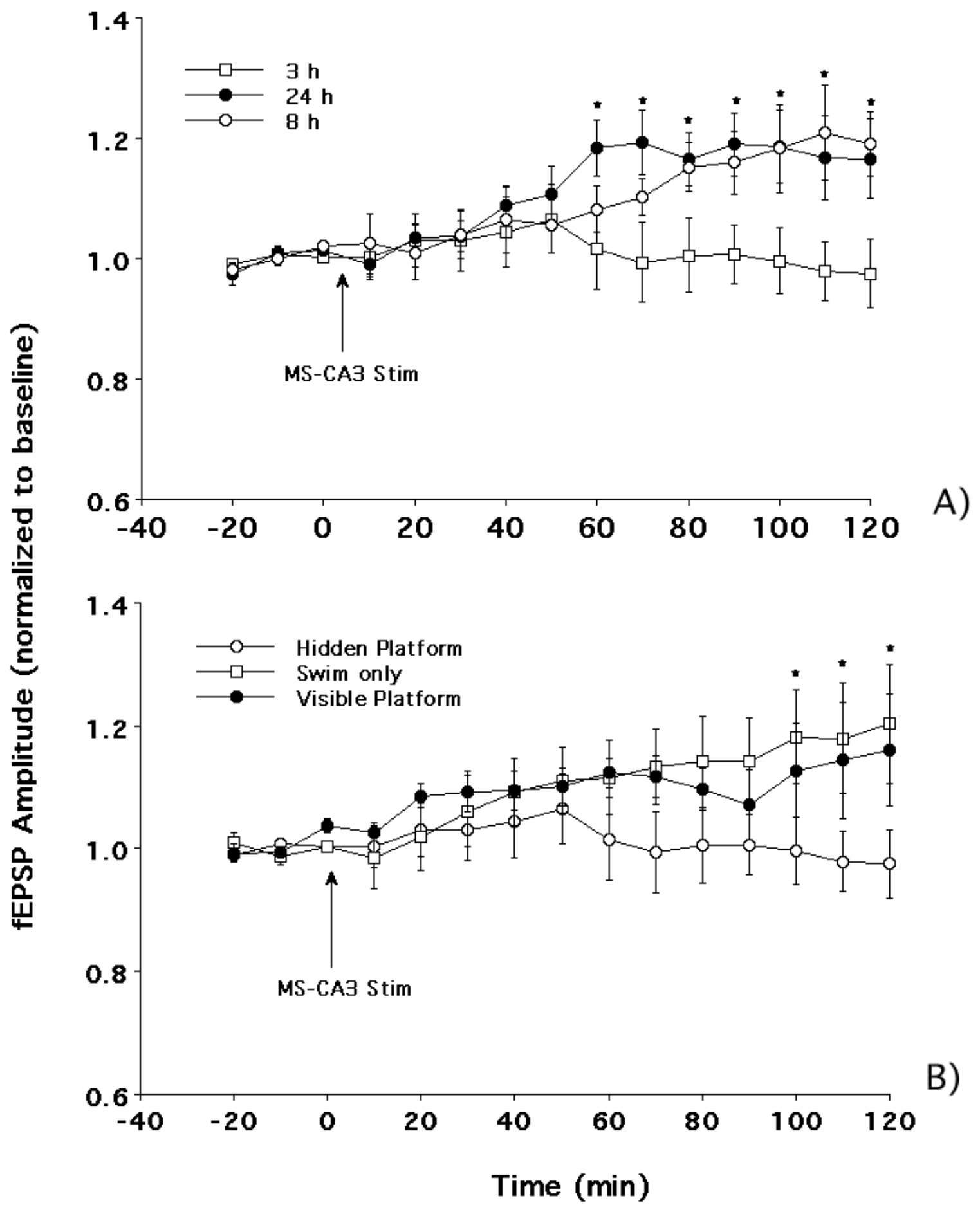


Figure 12. Effects of water maze training on MS-H LTP in the urethane-anesthetized preparation.

A) Alternating MS and CA3 stimulation (at arrow; 50 pulses each, $1000 \mathrm{~ms}$ inter-stimulus interval, $0.5 \mathrm{~Hz})$ delivered either $8 \mathrm{~h}(n=6)$ or $24 \mathrm{~h}(n=7)$ after the end of training on the hidden platform task enhanced fEPSP amplitude in CA1, while the MS-CA3 stimulation protocol $3 \mathrm{~h}$ $(n=7)$ after training failed to show this enhancement of fEPSP amplitude. Statistics: ANOVA comparing rats tested either 3,8 , or 24 h post training; effect of group $F_{2,17}=2.0, P=0.157$, effect of time, $F_{14,238}=6.5, P<0.0001$, group by time interaction, $F_{28,238}=2.6, P<0.0001 ; 3 \mathrm{vs}$. $24 \mathrm{~h}$, post training, effect of group $F_{1,12}=3.2, P=0.095$, effect of time, $\mathrm{F}_{14,168}=3.1, P<0.0001$, group by time interaction, $F_{14,168}=4.3, P<0.001 ; 3$ vs. 8 h, effect of group $\mathrm{F}_{1,11}=2.3, P=$ 0.156, effect of time, $\mathrm{F}_{14,154}=1.9, P=0.024$, group by time interaction, $\mathrm{F}_{14,154}=3.2, P<0.001$; 24 vs. 8 h, effect of group $\mathrm{F}_{1,11}=0.1, P=0.718$, effect of time, $\mathrm{F}_{14,154}=9.9, P<0.0001$, group by time interaction, $\mathrm{F}_{14,154}=0.71, P=0.760$. Simple effects of time significant for rats receiving MS-CA3 stimulation at $8 \mathrm{~h}\left(\mathrm{~F}_{14,70}=3.7, P<0.0001\right)$ and $24 \mathrm{~h}\left(\mathrm{~F}_{14,84}=7.4, P<0.0001\right)$, but not at $3 \mathrm{~h}\left(\mathrm{~F}_{14,84}=0.6, P=0.845\right)$ after training. B $)$ The effect of MS-CA3 stimulation delivered $3 \mathrm{~h}$ after training in the hidden platform version (same $3 \mathrm{~h}$ group as in panel $\mathbf{A}$ ), the visible platform version of the task $(n=7)$, and swim only controls $(n=7$, yoked control rats that swam for the same time per trial as each rat in the hidden platform group). While rats trained on the hidden platform task showed no enhancement of fEPSP amplitude (see above), animals trained on the visible platform version task and swim controls both showed fEPSP enhancement following alternating MS-CA3 stimulation (at arrow). Statistics: ANOVA comparing fEPSP amplitude in rats after hidden platform training, visible platform training, and swim controls; effect of group $\mathrm{F}_{2,18}=1.2$, $P=0.297$, effect of time, $\mathrm{F}_{14,252}=3.6, P<0.001$, group by time interaction, $\mathrm{F}_{28,252}=1.5, P$ 
$=0.046$; visible vs. hidden platform: effect of group $\mathrm{F}_{1,12}=2.2, P=0.11$, effect of time, $\mathrm{F}_{14,168}=$ $1.3, P=0.212$, group by time interaction, $\mathrm{F}_{14,168}=1.4, P=0.143$; hidden vs. swim only; effect of group $\mathrm{F}_{1,12}=1.7, P=0.211$, effect of time, $\mathrm{F}_{14,168}=2.0, P=0.014$, group by time interaction, $\mathrm{F}$ $14,168=2.8, P=0.001$. Simple effects of time significant for rats receiving MS-CA3 stimulation 3 $\mathrm{h}$ after training on visible platform $\left(\mathrm{F}_{14,84}=1.9, P=0.039\right)$ and swim only controls $\left(\mathrm{F}_{14,84}=3.6\right.$, $P<0.0001) . *$ denote significant $(P<0.05$; simple effect tests) group differences. 
Subsequently, we assessed the effects of alternating MS-CA3 stimulation $3 \mathrm{~h}$ after training on the visible platform task and in yoked control rats that swam for the same time per trial as each rat in the hidden platform group (swim only, Fig. 12B). In contrast to the occlusion effect noted $3 \mathrm{~h}$ after hidden platform training (see above), swim only controls showed a clear enhancement of fEPSP amplitude over a two-hour recording period following MS-CA3 stimulation (Fig. 12B; average fEPSP amplitude of $119 \%$ of baseline in the last 30 min of the recording period). Thus, it appears that stress and other factors associated with swimming in the maze do not account for the occlusion of MS-H LTP seen in rats trained in the hidden platform version of the task. Rats that received the stimulation protocol $3 \mathrm{~h}$ after training in the visible platform version of the task also showed an enhancement of fEPSP amplitude following MS-CA3 stimulation (115\% of baseline). However, statistically, this group of animals did not differ from either the hidden platform or the swim only groups (see caption for Fig. 12).

The experimental evidence summarized above suggests that MS-H LTP is occluded $3 \mathrm{~h}$ after hidden platform training, but not in the other experimental conditions ( 8 and $24 \mathrm{~h}$ after hidden platform, swim controls and visible platform training). These data suggest that hidden platform training results in the induction of an endogenous form of MS-H LTP during the earlierto-intermediate stages of memory consolidation (around $3 \mathrm{~h}$ ). Given this hypothesis, we analyzed whether baseline fEPSP amplitude shows differences between rats that display occluded versus intact MS-H LTP following delivery of the stimulation protocol. For this analysis, all experimental groups expressing MS-H LTP ( 8 and $24 \mathrm{~h}$ after hidden platform, swim controls, visible platform training) were pooled and their input/output curves (collected prior to the baseline recordings for the LTP experiment) compared to subjects showing MS-H LTP occlusion ( $3 \mathrm{~h}$ after hidden platform training). All animals showed increasing average fEPSP amplitude in 
CA1 with increasing stimulation intensity applied to the CA3 area (range of $0.1-1.0 \mathrm{~mA}$; Fig 13). Further, there was no significant difference in fEPSP amplitude between rats that subsequently showed synaptic enhancement in response to MS-CA3 stimulation and those in which enhanced fEPSP amplitude was occluded (note, however, that there appeared to be a trend toward larger fEPSP amplitude in occluded rats at the lower intensities of the input-output curve). 


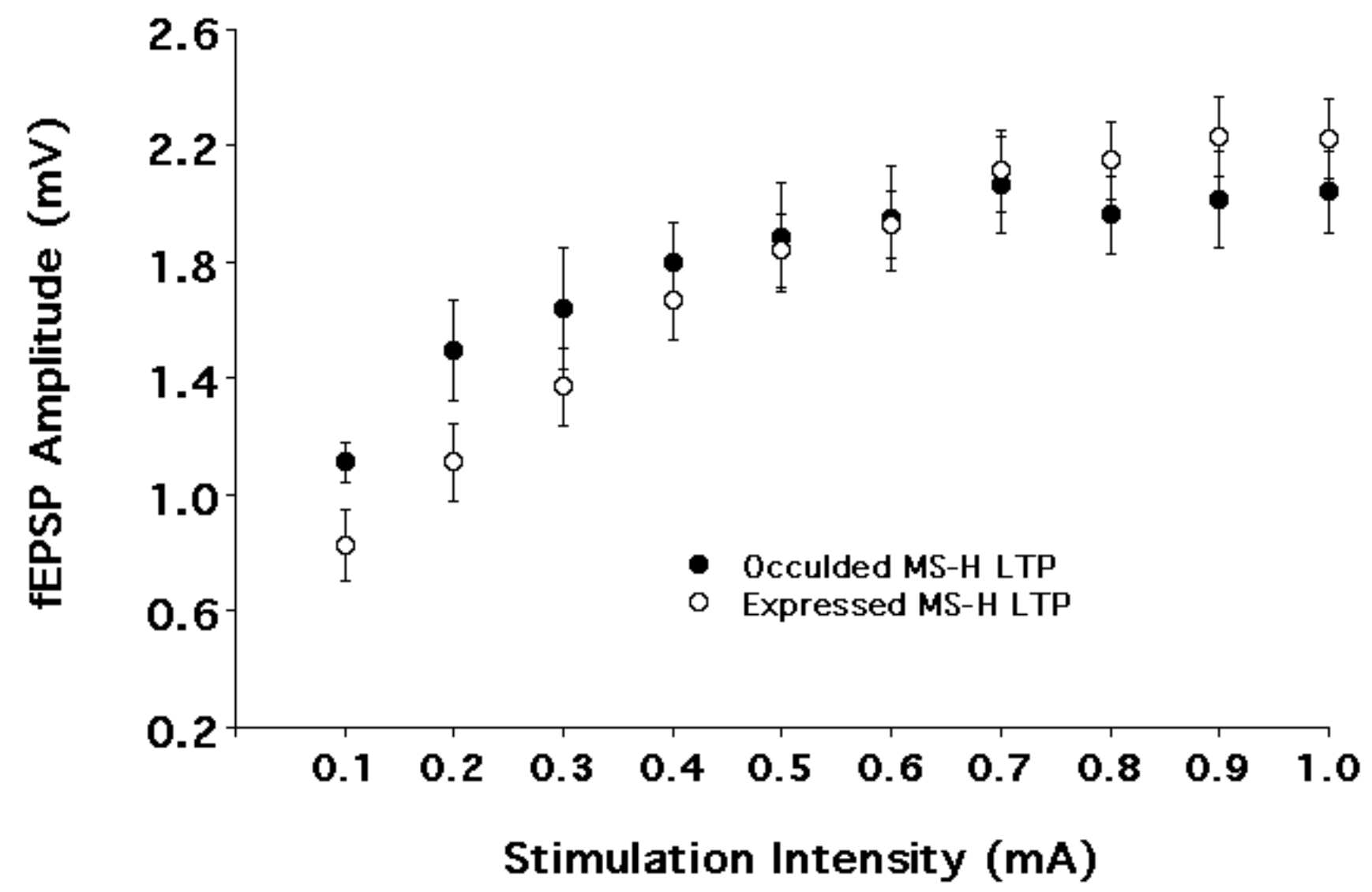

Figure 13. Input/output curves of rats exposed to water maze training.

Average fEPSP amplitude in CA1 in response to single pulse stimulation to CA3 at various stimulation intensities prior to acquisition of baseline recordings. This baseline input/output analysis was compared between all animals that subsequently showed enhanced fEPSP amplitude in response to MS-CA3 stimulation (i.e., MS-H LTP; $n=20$ ) and those in which MS-H LTP was occluded $(n=7)$. Average fEPSP amplitude in CA1 increased similarly in both groups with increasing stimulation intensity. There was a significant main effect of stimulation intensity $\left(\mathrm{F}_{9,288}\right.$ $=30.6, \mathrm{P}<0.001)$, but no other significant main or interaction effects $(\mathrm{P}$ 's $>0.05)$. 


\subsection{Discussion}

The majority of studies to date that have examined age-related changes in LTP and LTPlike synaptic enhancement have used various HFS protocols (e.g., 100-200 Hz) for the induction of LTP in the neocortex and hippocampal formation. Previously, we characterized a novel form of long-lasting synaptic enhancement induced by alternating LFS $(1 \mathrm{~Hz})$ to the MS and CA3 area of the hippocampus (MS-H LTP; Habib and Dringenberg, 2009; in press). However, age-related changes and the functional significance of MS-H LTP have never been examined. Here, we demonstrate that similar to LTP induced by HFS, low frequency-induced MS-H LTP is compromised in early aging, while similar levels of potentiation are expressed in the juvenile and adult hippocampus in vivo. Further, in adult rats, we show that MS-H LTP is occluded $3 \mathrm{~h}$ after training on the hidden platform version of the MWM, an effect not seen at 8 and $24 \mathrm{~h}$ after training.

To the best of our knowledge, the data demonstrating a loss of MS-H LTP in early aged rats are the first to characterize age-related changes in LTP induced by LFS in the intact brain preparation. The fact that MS-H LTP was compromised in 14 month-old rats, but remained intact in juvenile and adults, suggests that this form of LFS-LTP exhibits similarities to the agedependent changes of HFS-LTP known to occur in the hippocampus. For example, a series of reports have shown that LTP induced by HFS at CA3-CA1 synapses develops as early as postnatal day 16 and is expressed at maximal levels of potentiation by postnatal day 25 in vitro (Lauri et al., 2006; Abrahamsson et al., 2008). Here in juvenile rats (25-27 days), MS-CA3 stimulation induced gradual levels of enhancement in fEPSP amplitude that did not differ from 
adults and thus, exhibits full maturation around the same time as HFS-LTP. Additional evidence from a study that examined LFS-LTP following 1-Hz stimulation to local CA1 pyramidal cells in vitro showed that this form of potentiation is absent in pre-weaning rats (17-21 days), but fully developed at 25-days of age (Lanté et al., 2006b). These data suggest that some forms of LFSLTP in the hippocampus may be expressed at later stages of development relative to LTP induced by HFS that appears to mature by 16 days of age (Abrahamsson et al., 2008).

It is well established that aged animals show intact hippocampal LTP induction when robust, HFS protocols are used, but often display deficits in LTP maintenance (Barnes, 1979; Barnes and McNaughton, 1980; for review see Rosenzweig and Barnes., 2003). For example, earlier studies showed that multiple HFS induction episodes, delivered for several days, showed that aged animals displayed a greater rate of decay in LTP maintenance relative to younger adults (Barnes, 1979; Barnes and McNaughton, 1980). In contrast to HFS protocols, the LFS pattern used here was not effective in the induction of MS-H LTP in early aged rats. These findings are perhaps not surprising, given that the MS-CA3 stimulation is a weaker form of induction protocol than those typically used to elicit HFS-LTP in the aged hippocampus. Whether increasing the intensity and/or number of stimulation pulses to the MS or CA3 areas during LFS delivery would induce synaptic potentiation in CA1 is unknown.

The fact that stronger (i.e., HFS) and weaker (e.g., LFS of CA3 and MS) induction protocols differ in their ability of reveal age-related changes in hippocampal synaptic plasticity is consistent with previous work, which has used various induction protocols to induce LTP in rats of different ages. As previously mentioned, robust high intensity protocols are able to induce LTP in the aged hippocampus, however lower-intensity, "peri-threshold" stimulation protocols consistently result in age-related deficits in LTP induction (Deupree et al., 1993; Moore et al., 
1993; Rosenzweig et al., 1997). For example, delivery of HFS to Schaffer-collateral axons at lower intensities elicits greater levels of synaptic potentiation in CA1 in young animals relative to 24-month old-rats in vitro (Rosenzweig et al., 1997). These data also demonstrated an impaired temporal summation of multiple excitatory postsynaptic potentials in CA1 pyramidal cells during HFS, suggesting that postsynaptic cells in the aged hippocampus are less able to respond to multiple, high frequency inputs (Rosenzweig et al., 1997). An age-related decline in HFS-LTP has also been demonstrated by an increased threshold for LTP induction at CA3-CA1 and perforant path-dentate gyrus synapses (Rosenzweig et al., 1997; Bach et al., 1999; Barnes et al., 2000). Thus, "peri-threshold" HFS and LFS protocols like the one used here are both able to detect more subtle age-related deficits in hippocampal plasticity mechanisms.

It is well known that a reduction in the number and binding affinity of NMDA receptors in the hippocampus contributes to age-related decline in the induction of classical HFS-LTP (Magnusson, 1995; Shankar et al., 1998; Billard and Rouaud, 2007). Previous work has demonstrated that, similar to HFS-LTP, MS-H LTP requires NMDA receptor activation, protein kinase A activity, and subsequent protein synthesis (Malenka and Nicoll, 1999; Habib and Dringenberg, 2009). Thus, it is probable that changes to the NMDA receptor itself and/or other steps of the signaling pathways downstream from the NMDA receptor may contribute to the deficit of MS-H LTP seen here. At present, direct support for this assumption is lacking, but the use of receptor binding assays might allow for its assessment.

In contrast to the age-dependent deficits in MS-H LTP and classic LTP induced by HFS, Huang and Kandel (2006) characterized an age-dependent increase in long-lasting synaptic enhancement elicited by brief paired-pulse stimulation ( 2 pulses with an interpulse stimulus of 30 ms, pulse pairs repeated at $1 \mathrm{~Hz}$ ) of local CA1 pyramidal cells in vitro. Interestingly, this form of 
LTP was enhanced in the aged (24 month) hippocampus, providing a rare example of plasticity facilitation with increasing age.

The majority of protocols used to induce various types of LFS-LTP involve a stimulation frequency of $\sim 1 \mathrm{~Hz}$, a pattern of activity that is similar to endogenous cortical and hippocampal oscillations during slow wave sleep and has been suggested to promote sleep-related memory consolidation (Steriade et al., 1993; Buzsáki, 1998; Li et al., 2001; Sirota and Buzsáki, 2005; Born et al., 2006; Lante' et al. 2006a,b; Wolansky et al., 2006; Huang and Kandel, 2007; Schall et al., 2008; Habib and Dringenberg, 2009; in press). Thus, we have previously raised the question whether LFS-LTP may provide one of the mechanisms mediating the influence of endogenous slow oscillations on synaptic plasticity during sleep in the mammalian brain (Buzsáki, 1989; Sipas and Wilson, 1998; Habib and Dringenberg, 2010). Interestingly, aged human subjects and rodents show an overall reduction in slow wave sleep and cortical slow oscillations, changes in sleep patterns that are thought to contribute to deficits in age-related memory consolidation (Van Gool and Mirmiran, 1983; Hornung et al., 2005). Thus, the overall reduction in slow wave sleep (and related $\sim 1 \mathrm{~Hz}$ activity) and the deficient induction mechanisms mediating slow wave activity-related synaptic potentiation may both play a role in the impairments in sleep-related memory consolidation thought to occur with aging.

While we have previously raised the possibility that LFS-LTP may play a role in memory consolidation processes, the functional significance of MS-H LTP has never been examined. Thus, in the second set of experiments, we studied the influence of behavioural water maze training, a form of learning known to be dependent on hippocampal circuitry (Morris, 1981; Morris et al., 1986), on the subsequent expression of MS-H LTP. Electrophysiological procedures were carried out so that the delivery of the MS-CA3 stimulation protocol occurred exactly at 3,8, 
or $24 \mathrm{~h}$ after the end of behavioural training. Alternating MS-CA3 stimulation delivered at $8 \mathrm{~h}$ or $24 \mathrm{~h}$ after the end of training on the hidden platform task resulted in an enhancement of fEPSP amplitude in CA1 to levels equivalent to those seen in the absence of training (Habib and Dringenberg, 2009; in press). Remarkably, however, rats that received the protocol $3 \mathrm{~h}$ after training failed to show the typical enhancement in fEPSP amplitude normally seen with MS-CA3 stimulation. Conversely, yoked control rats that swam for the same time per trial as each rat in the hidden platform group expressed MS-H LTP, suggesting that stress and other factors associated with swimming in the maze do not account for the occlusion of MS-H LTP seen in rats 3 hours after hidden platform training.

These results are the first to demonstrate an occlusion of LTP at hippocampal synapses elicited by LFS patterns in vivo. Similar to our observations, several recent investigations have demonstrated that learning/training experiences can occlude or minimize subsequent induction of LTP elicited by HFS in subcortical and neocortical areas (hippocampus; Whitlock et al., 2006; motor cortex; Rioult-Pedotti et al., 2000; lateral amygdala; Rogan et al., 1997). Further, behavioural training has been shown to enhance baseline changes in synaptic responses in the motor cortex and hippocampus, a training-induced synaptic facilitation that was not observed in the current experiments (Whitlock et al., 2006; Rioult-Pedotti et al., 2000). Here, baseline fEPSP amplitude did not differ significantly between rats that displayed occluded versus intact MS-H LTP. This apparent discrepancy might, at least in part, be related to different recording methodologies. For example, the enhancement in baseline fEPSP amplitude in the hippocampus following inhibitory avoidance learning was detected using a multielectrode recording array, allowing the simultaneous measurement of synaptic strength in a larger part of the CA1 field (Whitlock et al., 2006). Perhaps the use of only one recording electrode in the current experiment 
was insufficient to detect changes at different locations within the diffuse dendrites of the CA1 pyramidal cells. Nevertheless, there appeared to be a trend toward larger baseline fEPSP amplitude at lower stimulation (0.1- $0.5 \mathrm{~mA})$ intensities in rats that displayed occlusion relative to those with intact MS-H LTP. The use of more sophisticated multi-electrode arrays might increase the probability of detecting changes in training-induced strength at widely distributed synapses throughout the hippocampal network.

In order to determine whether the training-induced occlusion seen here, was specific to the hidden platform version of the task, we delivered the LFS protocol in rats 3 hours after training on a visible platform in the MWM. Rats trained on this task, showed an enhancement of fEPSP amplitude following MS-CA3 stimulation, however, statistically, this group of animals did not differ from either the hidden platform groups (showing occlusion) or the swim only animals, which showed intact MS-H LTP. This lack of a significant difference from both of these groups may be attributed to the fact that levels of potentiation in the visible platform group were highly heterogeneous, with three to four animals in this group demonstrating an occlusion and intact MS-H LTP, respectively. A possible explanation for this variability might be that at least some rats trained to swim to the visible platform actually used spatial, hippocampal-dependent strategies to locate the platform. These findings coincide with previous reports demonstrating residual impairments in performance on the visible platform version of the MWM task in hippocampal-lesioned subjects (Morris, 1981; Czurkó et al., 1997; Cassel et al., 1998; de Bruin et al., 2001). Further, in series of elegant experiments, McDonald and White $(1993 ; 1994)$ showed that animals can learn to acquire the visible platform using two different learning strategies. Animals can accomplish the task by either learning the location of the visible platform and its relation to fixed distal cues around the maze (place strategy; hippocampal-dependent learning) or 
through acquisition of a simple stimulus (platform)-response (escape) learning to approach the visible platform (cue strategy; striatal-dependent learning). They further demonstrated that individual animals typically prefer one or the other of these strategies during training. Thus, it is not surprising that some animals trained on the visible platform expressed occlusion in MS-H LTP, since they may have resorted to hippocampal-dependent, spatial strategies during learning (McDonald and White, 1994).

The fact that rats show occlusion at $3 \mathrm{~h}$ after hidden platform training suggests that this form of learning induced an endogenous form of MS-H LTP during memory consolidation in local hippocampal circuits. At the neuronal level, earlier stages of consolidation involve local changes in cellular events (up to several hours) that ultimately translate to a 'systems' level reorganization of synaptic connectivity between multiple brain structures (involving days, weeks or years; Martin and Clarke, 2007). The occlusion effect found here has clear, temporal limits, since MS-H LTP recovers and is expressed at 8 and $24 \mathrm{~h}$ after training, suggesting that this form of synaptic enhancement may mediate earlier cellular changes involved in memory consolidation. It is thought that NMDA receptor activity is one of the cellular mechanisms that contributes to early stabilization of memory after an initial encoding event (for review see Lynch, 2004; Abraham and Williams, 2008). For example, bilateral hippocampal infusions of a NMDA receptor antagonist administered immediately after inhibitory avoidance training blocks retention measured $24 \mathrm{~h}$ after learning (Bevilaqua et al., 2005; Roesler et al., 2005), findings that have been extended to training on the hidden platform version of the MWM task (McDonald et al., 2005). The fact that MS-H LTP relies on NMDA receptor activity for its induction reinforces the notion that this form of synaptic potentiation may promote mechanisms that mediate early stages of consolidation of hippocampal-dependent learning experiences (Habib and Dringenberg, 2009). 
Future studies will examine the effects of NMDA receptor antagonists, administered immediately after training, on the occlusion of MS-H LTP on the hidden platform version of the MWM task. The main objective of this experiment will be to determine whether NMDA receptor blockade reverses the occlusion effects characterized here, which would provide further understanding of the mechanistic changes thought to contribute to hippocampal-dependent memory consolidation. Further, the fact that MS-H LTP is impaired in early aging suggests an age-related deficit in mechanisms of consolidation. Consequently, future work testing this hypothesis will examine whether the deficit in MS-H LTP translates into consolidation impairments in early-aged rats. In this proposed experiment, it would be of interest to determine whether early aged animals perform poorly on a memory retention test (i.e., probe trial) 3 hours after training in the hidden platform task.

The new and exciting field of LFS-LTP has raised several important questions regarding its function, mechanisms, and its relation to well-known classical forms of HFS-LTP. Here, in two separate experiments, we characterized age-related changes of a novel form of LFS-induced synaptic potentiation (MS-H LTP) in vivo and secondly, investigated its functional significance in early stages of hippocampal-dependent consolidation in adult animals. Collectively, these data provide further understanding of the function and characteristics of this specific type of LFS-LTP and provide a new perspective on the study of synaptic plasticity in relation to learning and memory functions in the mammalian forebrain. 


\section{Chapter 6. General Discussion}

\subsection{Summary of main findings}

Long-term potentiation (LTP) and long-term depression (LTD) are two highly intensely studied forms of synaptic plasticity which are thought to play a functional role in learning and memory processes. It is generally assumed that the direction of synaptic change, either up- or down regulation of synaptic strength, is determined by specific patterns of afferent inputs, with high- and low frequency stimulation inducing LTP and LTD, respectively (Massey and Bashir, 2007; Raymond, 2007). In the current thesis, I challenge this dogma of "high frequency stimulation (HFS)-LTP and low frequency stimulation (LFS)-LTD” by providing evidence demonstrating LFS-induced synaptic potentiation in the rodent hippocampus.

My first set of experiments shows that alternating, single pulse stimulation $(1 \mathrm{~Hz})$ of the MS and CA3 hippocampal $(\mathrm{H})$ commissural fibres results in synaptic enhancement of field excitatory potentials (fEPSPs) recorded in area CA1 of urethane-anesthetised rats (MS-H-LTP). This MS-H LTP is long lasting ( $>5 \mathrm{~h})$, requires a specific inter-stimulus interval ( $1 \mathrm{~s}$ between successive MS and CA3 stimulation pulses), saturates with repeated stimulation episodes and depends on NMDA, but not cholinergic receptor activation (Chapter 2). These reports are the first to document long-lasting synaptic enhancement in response to low frequency activity patterns in vivo and reinforce other LFS-LTP phenomena characterized in hippocampal and amygdalar circuits in vitro (Lante' et al., 2006a,b; Huang and Kandel, 2007). In a review paper (Chapter 3), I raise the question whether LFS protocols provide a means to more accurately mimic some endogenous, oscillatory activity patterns $(\sim 1 \mathrm{~Hz})$ present in hippocampal and neocortical circuits 
during sleep, which have been proposed to play a role in sleep-related memory consolidation. Moreover, I compare the well-known synaptic and cellular mechanisms underlying classical, HFS-LTP to those mediating MS-H LTP, as well as several other, recently discovered types of LFS-LTP in the hippocampus and amygdala in vitro. Subsequently, I characterize the cellular mechanisms of MS-H LTP and their similarity to classical HFS-LTP by means of drug application through reverse microdialysis at the CA1 recording site and show that MS-H LTP depends on protein kinase A and protein synthesis (Chapter 4). This surprising similarity between mechanisms mediating classical HFS-LTP and MS-H LTP is further supported by occlusion experiments whereby LFS and HFS episodes, delivered to the same animal, compete for the available synaptic potentiation of CA3-CA1 synapses. Lastly, for my final study (Chapter 5), I carried out two separate experiments assessing 1) age-related changes of MS-H LTP and 2) the effects of water maze training on this form of LTP in adult rats. Here, I initially demonstrate that, similar to LTP induced by HFS, low frequency-induced MS-H LTP is compromised in early aging, while similar levels of potentiation are expressed in the juvenile and adult hippocampus. In the second set of experiments, alternating MS-CA3 stimulation was delivered exactly at 3, 8, or $24 \mathrm{~h}$ after the end of behavioural (water maze) training and results show that MS-H LTP is occluded $3 \mathrm{~h}$ after training, while it remains intact at 8 and $24 \mathrm{~h}$ intervals. These experiments are consistent with a possible role of MS-H LTP in early stages of consolidation of spatial reference memory in hippocampal circuits.

For a more detailed discussion of the main findings of the current thesis, the reader is encouraged to refer to the corresponding chapters indicted above. For the purpose of this general discussion, I will highlight important questions regarding the future and the direction of study of 
MS-H LTP in the mammalian brain. Specifically, I will address four main questions of interest and give a brief overview of methodologies that may be used in the proposed experiments.

\subsection{The future of LFS-induced MS-H LTP}

\subsubsection{Can MS-H LTP be expressed in freely moving animals?}

As mentioned throughout this thesis, the majority of experimental protocols used the slice preparation to study forms of LFS-LTP in the amygdala and hippocampus (e.g., Li et al., 2001; Lante'et al. 2006a,b; Huang and Kandel, 2007), while fewer studies have shown LFS-LTP phenomena in urethane-anesthetized animals (Habib and Dringenberg, 2009; in press). The study of LTP in slices has some advantages over the anaesthetized preparation, such as direct visualization of brain structures allowing for more accurate electrode placements, and precise control over experimental conditions such as temperature, $\mathrm{pH}$ and drug concentrations (Hölscher, 1997). However, the slice preparation eliminates modulatory processes known to influence synaptic plasticity, such as serotonergic afference from the raphe nuclei, cholinergic innervation from the basal forebrain, and modulation by hormones and neurotrophic factors (Hölscher, 1997). Given the fact that the anesthetised preparation allows for the influence of these endogenous mechanisms, it can be considered a more valid model for the study of various forms of synaptic plasticity in the nervous system. Nevertheless, the strong, inhibitory effects exerted by anaesthetics might disrupt endogenous brain activities and, consequently, alter plasticity phenomena in the brain (Riedel et al., 1994). Thus, one might argue that the intact, nonanesthetized nervous system of freely behaving animals provides the most optimal environment for the study of behavior-related forms of synaptic plasticity. Interestingly, a series of experiments in the hippocampus has characterized HFS-induced LTP in freely moving rats, some 
of which show a synaptic enhancement lasting for up to at least one year (e.g., Stäubli and Scafidi, 1999; Abraham et al., 2002; Leung et al., 2003; Dringenberg et al., 2008).

The question of whether or not MS-H LTP or other forms of LFS-LTP can be expressed in freely moving rats has never been examined. Future experiments will address this main question by comparing the effects characterized under urethane to MS-H LTP in freely moving animals using the same MS-CA3 alternating stimulation protocol described throughout the thesis. The use of a freely moving preparation will allow for a more detailed examination of the longlasting nature of MS-H LTP, as well as its functional significance in learning and memory processes.

\subsubsection{Do activity patterns similar to the MS-CA3 induction protocol occur endogenously in the rat brain?}

A prominent feature of hippocampal activity is the prevalence of several, distinct oscillatory patterns that are tightly linked to specific behavioural states and exert important influences over the expression of synaptic plasticity (Buzsáki, 2002; Hyman et al., 2003; Axmacher et al., 2006). The importance of developing LTP induction protocols that mimic some of these endogenous hippocampal activity patterns was addressed shortly after the discovery of LTP in the hippocampal formation (see; Bliss and Lómo, 1973; Larson et al., 1986; Stäubli and Lynch, 1987; Albensi et al., 2007). For example, theta-burst stimulation (TBS), a commonly used and highly effective LTP induction protocol, mimics theta $(3-12 \mathrm{~Hz})$ activity present in the hippocampus during specific types of behavior (walking, postural adjustments, head movements, REM sleep, “Type 1 behavior’; Vanderwolf, 1969, 1988; Buzsáki, 2002). Another highly regular, slow oscillatory pattern of $1 \mathrm{~Hz}$ has recently been characterized in the hippocampus of sleeping and anesthetized rats (Wolansky et al. 2006; Schall et al., 2008), activity that parallels 
the slow, neocortical oscillations present in slow wave sleep (Steriade et al., 1993). It has been suggested that this phasic relation of synchronous slow oscillatory patterns between the hippocampus and neocortex facilitates mechanisms of sleep-related memory consolidation (see Sipas and Wilson, 1998; Wolansky et al., 2006). Interestingly, these endogenous activity patterns occur at frequencies overlapping with those used to induce MS-H LTP and other forms of LFSLTP phenomena. In fact, an interstimulus interval of one second between MS and CA3 stimulation is critical in the induction of MS-H LTP at CA1 pyramidal cells (see chapter 2). However, whether there is an endogenous, phasic relation of activity between the MS and CA3 area that mimics this electrical $1 \mathrm{~Hz}$ stimulation pattern is unknown.

Consequently, future experiments could involve simultaneous recordings of EEG activity in the MS and CA3 area by means of chronically implanted recording electrodes in freely moving rats. These simultaneous patterns of EEG activity will be recorded and analyzed in the rat during the following behavioural states: awake-immobility, walking, slow-wave sleep and REM sleep. Since EEG activity patterns in the hippocampus are expressed at higher frequencies during walking and REM sleep (Vanderwolf, 1969, 1988; Buzsáki, 2002; Leung et al., 2003), it is expected that any potential, phasically related slow rhythmic activity in the MS and CA3 would only be present during periods of slow-wave sleep.

Previously, I have shown that MS-H LTP is occluded $3 \mathrm{~h}$ after training on the hidden platform version of the Morris water maze (MWM) task (chapter 5), which suggests that this type of learning induced an endogenous form of MS-H LTP during memory consolidation in local hippocampal circuits. Any evidence of simultaneous patterns of endogenous slow oscillatory activity $(\sim 1 \mathrm{~Hz})$ in the hippocampus and MS during specific behavioural states would raise the question of whether this phasic relation is present during the time window of up to 3 hours after 
hidden platform training in the MWM (i.e., during occlusion of MS-H LTP). To address this question, future EEG experiments (same as above) will be carried out in separate groups of rats immediately after undergoing the following experimental conditions: 1) training on the hidden platform of the MWM task 2) visible platform training and 3) swim only conditions. Here, it would be of interest to compare the relation of EEG patterns between the MS and CA3 area in all three groups of animals. Moreover, given the fact that MS-H LTP is no longer occluded at 8 and $24 \mathrm{~h}$ after hidden platform training, it would also be of interest to see how simultaneous MS and CA3 endogenous rhythmic activity changes in rats trained on the hidden platform task at various intervals following training.

\subsubsection{Do alternating LFS patterns of $1 \mathrm{~Hz}$ effectively induce LFS-LTP in areas outside the hippocampus?}

It is evident that alternating LFS applied to the MS and CA3 area is effective in the induction of long-lasting synaptic enhancement at CA1 pyramidal cells. Whether repeated, low frequency $(1 \mathrm{~Hz})$ co-activation of separate afferent systems to areas outside the hippocampus is effective in the induction of LTP-like phenomena has never been examined in vivo. However, there are some types of HFS-LTP that require the integration of separate synaptic inputs for their induction (Popescu et al. 2007; in press). For example, Popescu et al. (2007) characterized a form of long-lasting heterosynaptic facilitation ( $>30 \mathrm{~min}$ ) induced by pairing of corticostriatal and amygdala glutamatergic inputs onto single, medium spiny neurons in the striatum in vitro. This corticostriatal synaptic enhancement is NMDA receptor-dependent and is elicited by coactivation of separate afferent fiber systems, mechanisms of induction that overlap with those required for MS-H LTP (Popescu et al., 2007; in press). Given this apparent similarity, it would 
be of interest to examine whether the alternating LFS protocol used to elicit MS-H LTP in the hippocampus is similarly effective in the induction of LFS-LTP at corticostriatal synapses.

To address this question, future studies will be carried out in urethane-anesthetised rats using the electrode placements employed in vitro by Popescu et al. (2007; in press) and the stimulation parameters (alternating $1 \mathrm{~Hz}$ stimulation) used to induce MS-H LTP. Electrode placements will consist of three stimulation electrodes, one in the basolateral amygdala (BLA) and two in the temporal cortex, and one recording electrode will be used to measure field excitatory postsynaptic potential (fEPSP) amplitude in the ventral striatum. Recordings of striatal fEPSP amplitude will be examined before (baseline) and up to four hours after delivery of alternating LFS to the BLA and the temporal cortex (a total of 50 pulses at $0.5 \mathrm{~Hz}$ for each, starting with BLA and then temporal cortex stimulation, 1,000 ms interstimulus interval).

The study of LFS-LTP induced by co-activation of heterosynaptic afferent fibers in distinct areas outside the hippocampus may provide a means to examine synaptic mechanisms of consolidation of hippocampal-independent learning tasks in the mammalian brain. Any evidence of a form of "striatal LFS-LTP" elicited by alternating LFS to the BLA and temporal cortex (see above) would raise the following interesting question:

\subsubsection{Do forms of LFS-LTP mediate early consolidation of striatal-dependent learning tasks?}

As previously mentioned, hippocampal-dependent training on the hidden platform version of the MWM task occludes MS-H LTP (chapter 5), suggesting that certain forms of LFSLTP promote mechanisms of consolidation of hippocampal-dependent spatial memory. Whether this notion holds true for non-hippocampal dependent learning tasks is unknown. It appears that the activation of the BLA (via local drug infusions) immediately after training on the visible 
platform of the MWM improves retention 24 hours after training. Evidently, the amygdala facilitates training on the visible platform, a task known to dependent on the striatum and, thus presumably facilitates striatal plasticity (Packard, 1994). Based on these findings, and other similar work (McDonald and White, 1993) highlighting the importance of the striatum in cue response learning, the following proposed experiment will examine the functional significance of “striatal LFS-LTP” elicited by LFS of the BLA and temporal cortex following water maze training. Specifically, these experiments will compare the already characterized occlusion effects of MS-H LTP to occlusion of striatal LFS-LTP following training on either the hidden or visible platform version of MWM task. An additional group of "swim only" animals will be allowed to swim for the same time per trial as rats trained on the hidden and visible platform and will control for effects of stress that may influence the expression of both forms of LTP under investigation. In accordance with previous experimental methods (chapter 5), water maze training on both the hidden and visible platform will consist of 3 blocks of 4 trials ( 5 min inter-block period) and all subsequent electrophysiological procedures will be carried out in the intact urethane preparation. All animals trained on the hidden and visible platform will receive either low frequency "BLA-temporal stimulation" or MS-CA3 stimulation. Electrode placements in rats receiving the "BLA-temporal stimulation" will consist of two stimulation electrodes (one in the following areas: BLA and temporal cortex) and one recording electrode in the ventral striatum. In this group of animals the "BLA-temporal cortex" stimulation protocol will be delivered in individual animals at exactly 3 hours after either hidden or visible platform training. For the induction of "striatal LFS-LTP" alternating LFS will be delivered to the BLA and the temporal cortex (a total of 50 pulses at $0.5 \mathrm{~Hz}$ for each, starting with BLA and then temporal cortex stimulation, 1,000 ms interstimulus interval). The same MS-CA3 stimulation previously 
described will be used to induce MS-H LTP in separate group of rats at exactly 3 hours after either hidden or visible platform training. Recordings of striatal or CA1 fEPSP amplitude will be examined before and up to four hours after delivery of the corresponding alternating LFS protocols.

Given the fact that there is a dissociation between the roles of the hippocampus and striatum in place versus cue strategy learning, respectively (McDonald and White, 1993; 1994; Packard et al., 1994), one would expect to see occlusion of MS-H LTP in rats trained on the hidden platform, accompanied by intact "striatal LFS-LTP" in animals receiving the "BLAtemporal cortex stimulation" after hidden platform training. Conversely, rats trained on the visible platform version of the task should predominately show intact MS-H LTP, accompanied by an occlusion of striatal LFS-LTP in animals receiving "BLA-temporal cortex stimulation" 3 hours after visible platform training. However, it is evident that animals can accomplish the visible platform task by using either hippocampal-dependent place or striatal-dependent cue strategies during learning. Thus, some rats may show an occlusion of MS-H LTP after visible platform training, an occlusion that, in theory, should co-exist with intact striatal LFS-LTP.

There is compelling evidence to suggest that LFS-LTP induced by alternating $1 \mathrm{~Hz}$ stimulation patterns promotes mechanisms of hippocampal-dependent consolidation in the mammalian brain (chapter 5). Perhaps the occlusion of a form of "striatal LFS-LTP" induced by these alternating LFS patterns may provide a mechanism by which the BLA modulates plasticity at corticostriatal synapses thought to occur during consolidation of striatal-dependent learning tasks (e.g., motor and cue-response learning; see Lovinger, 2010; Pennartz et al., 2009). Future work in the emerging field of LFS-LTP at cortical and subcortical synapses may provide an 
additional avenue for the study of cellular mechanisms mediating synaptic plasticity thought to occur during memory consolidation.

\subsubsection{Conclusions}

The current thesis characterizes a novel form of LFS-induced hippocampal plasticity (MS-H LTP) in the urethane intact brain preparation. These series of experiments utilize cellular, electrophysiological and behavioural measures to provide a detailed analysis of MS-H LTP and its role in early consolidation of hippocampal-dependent learning tasks. These experiments, together with related work demonstrating LFS-LTP in various subcortical areas, make us question whether the "HFS-LTP and LFS-LTD" dogma fully captures the entire spectrum of synaptic changes in the mammalian brain. Based on the findings documented and discussed here, I now ask you, the reader: is low-frequency-induced synaptic potentiation a paradigm shift in the field of memory-related plasticity mechanisms? 


\section{References}

Abraham WC, Williams JM. 2008. LTP maintenance and its protein synthesis-dependence.

Neurobiol Learn Mem 89:260-268.

Abraham WC, Logan B, Greenwood JM, Dragunow M. 2002. Induction and experience-dependent consolidation of stable long-term potentiation lasting months in the hippocampus. J Neurosci 22:9626-9634.

Abrahamsson T, Gustafsson B, Hanse E. 2008. AMPA silencing is a prerequisite for developmental long-term potentiation in the hippocampal CA1 region. J Neurophysiol 100:2605-2614.

Ahmed T, Frey JU. 2005. Plasticity-specific phosphorylation of CaMKII, MAP-kinases and CREB during late-LTP in rat hippocampal slices in vitro. Neuropharmacology 49:477-492.

Albensi, BC, Oliver DR, Toupin J, Oderdo G. 2007. Electrical stimulation protocols for hippocampal synaptic plasticity and neuronal hyper-excitability: are they effective or relevant? Exp Neurol 204:1-13.

Amaral DG, Kurz J. 1985. An analysis of the origins of the cholinergic and noncholinergic septal projections to the hippocampal formation of the rat. J Comp Neurol 240:37-59.

Auerbach JM, Segal M. 1994. A novel cholinergic induction of long-term potentiation in rat hippocampus. J Neurophysiol 72: 2034-2040.

Axmacher N, Mormann F, Fernández G, Elger CE, Fell J. 2006. Memory formation by neuronal synchronization. Brain Res Rev 52:170-182.

Bach ME, Barad M, Son H, Zhuo M, Lu YF, Shih R, Mansuy I, Hawkins RD, Kandel ER. 1999. Age-related defects in spatial memory are correlated with defects in the late phase of hippocampal long-term potentiation in vitro and are attenuated by drugs that enhance the cAMP signalling pathway. Proc Natl Acad Sci USA 96:5280-5285. 
Barnes CA, Rao G, Houston FP. 2000. LTP induction threshold change in old rats at the perforant path-granule cell synapse. Neurbiol Aging 21:613-620.

Barnes CA, McNaughton BL. 1985. An age comparison of the rates of acquisition and forgetting of spatial information in relation to long-term enhancement of hippocampal synapses. Behav Neurosci 99:1040-1048.

Barnes CA, McNaughton BL. 1980. Spatial memory and hippocampal synaptic plasticity in middleaged and senescent rats. In: Stein D. (Ed.). Psychobiology of Aging: Problems and Perspectives. Elsevier, New York, pp. 253-272.

Barnes CA. 1979. Memory deficits associated with senescence: A neurophysiological and behavioral study in the rat. J Comp Physiol Psychol 93:74-104.

Bayazitov IT, Richardson RJ, Fricke RG, Zakharenko SS. 2007. Slow presynaptic and fast postsynaptic components of compound long-term potentiation. J Neurosci 27:11510-11521.

Bekkers JM. Stevens CF. 1990. Presynaptic mechanism for long-term potentiation in the hippocampus. Nature 346:724-729.

Bennett MR. 2000. The concept of long term potentiation of transmission at synapses. Prog Neurobiol 60:109-137.

Bergado JA, Almaguer W. 2002. Aging and synaptic plasticity: a review. Neural Plast 9: 217-232.

Bevilaqua LR, Medina JH, Izquierdo I, Cammarota M. 2005. Memory consolidation induces Nmethyl-D-aspartic acid-receptor- and Ca2+/calmodulin-dependent protein kinase II-dependent modifications in alpha-amino-3-hydroxy-5-methylisoxazole-4-propionic acid receptor properties. Neuroscience 136:397-403.

Bi GQ, Rubin J. 2005. Timing in synaptic plasticity: from detection to integration. Trends Neurosci 28:222-228. 
Billard JM, Rouaud E. Deficit of NMDA receptor activation in CA1 hippocampal area of aged rats is rescued by D-cycloserine. Eur J Neurosci 25:2260-2268.

Blair HT, Schafe GE, Bauer EP, Rodrigues SM, LeDoux JE. 2001. Synaptic plasticity in the lateral amygdala: a cellular hypothesis of fear conditioning. Learn Mem 8:229-242.

Bland BH, Declerck S, Jackson J, Glasgow S, Oddie S. 2007. Septohippocampal properties of Nmethyl-D-aspartate-induced theta-band oscillation and synchrony. Synapse 61:185-197.

Bliss TVP, Collingridge GL.1993. A synaptic model of memory: long-term potentiation in the hippocampus. Nature 361:31-39.

Bliss TVP, Lomo T. 1973. Long-lasting potentiation of synaptic transmission in the dentate area of the anaesthetized rabbit following stimulation of the perforant path. J Physiol 232:331-356.

Böhme GA, Bon C, Stutzmann JM, Doble A, Blanchard JC. 1991. Possible involvement of nitric oxide in long-term potentiation. Eur J Pharmcol 199:379-381.

Born J, Rasch B, Gais S. 2006. Sleep to remember. Neuroscientist 12:410-424.

Bramham CR, Srebro B. 1987. Induction of long-term depression and potentiation by low- and highfrequency stimulation in the dentate area of the anesthetized rat: magnitude, time course and EEG. Brain Res 405:100-107.

Bredt DS, Nicoll RA. 2003. AMPA receptor trafficking at excitatory synapses. Neuron 40:361-379.

Bresnahan EL, Wiser PR, Muth NJ, Ingram DK. 1992. Delayed matching-to-sample performance by rats in a new avoidance-motivated maze: response to scopolamine and fimbria-fornix lesions. Physiol Behav 51:735-746.

Buzsáki G. 2002. Theta oscillations in the hippocampus. Neuron 33:325-340.

Buzsáki G. 1998. Memory consolidation during sleep: a neurophysiological perspective. J Sleep Res 7:17-23. 
Buzsáki G. 1989. Two-stage model of memory trace formation: a role for "noisy" brain states. Neuroscience 31:551-570.

Buzsáki G, Leung LW, Vanderwolf CH. 1983. Cellular bases of hippocampal EEG in the behaving rat. Brain Res 287:139-171.

Caporale N, Dan Y. 2008. Spike timing-dependent plasticity: A Hebbian learning rule. Annu Rev Neurosci 31:25-46.

Cassel JC, Cassel S, Galani R, Kelche C, Will B, Jarrard L. 1998. Fimbria-fornix vs. selective hippocampal lesions in rats: effects on locomotor activity and spatial learning and memory. $69: 22-45$.

Christie BR, Kerr DS, Abraham WC. 1994. Flip side of synaptic plasticity: long-term depression mechanisms in the hippocampus. Hippocampus 4:127-135.

Christie BR, Abraham WC. 1992. NMDA-dependent heterosynaptic long-term depression in the dentate gyrus of anaesthetized rats. Synapse 10:1-6.

Colom LV, Castaneda MT, Reyna T, Hernandez S, Garrido-Sanabria E. 2005. Characterization of medial septal glutamatergic neurons and their projection to the hippocampus. Synapse 58:151164.

Czurkó A, Czéh B, Seress L, Nadel L, Bures J. 1997. Severe spatial navigation deficit in the Morris water maze after single high dose of neonatal x-ray irradiation in the rat. Proc Natl Acad Sci U S A $94: 2766-2771$.

Dan Y, Poo MM. 2004. Spike timing-dependent plasticity of neural circuits. Neuron 44:23-30.

Danik M, Puma C, Quirion R, Williams S. 2003. Widely expressed transcripts for chemokine receptor CXCR1 in identified glutamatergic, gamma-aminobutyric acidergic, and cholinergic neurons and 
astrocytes of the rat brain: a single-cell reverse transcription-multiplex polymerase chain reaction study. J Neurosci Res 74:286-295.

Dashniani MG, Beseliia GV, Maglakelidze GA, Burdzhanadze MA, Chkhikvishvili NT. 2009.Effects of the selective lesions of cholinergic septohippocampal neurons on different forms of memory and learning process. Georgian Med News 166:81-85.

de Bruin JP, Moita MP, de Brabander HM, Joosten RN. 2001. Place and response learning of rats in a Morris water maze: differential effects of fimbria fornix and medial prefrontal cortex lesions. $75: 164-178$.

Debanne D.1996. Associative synaptic plasticity in hippocampus and visual cortex: cellular mechanisms and functional implications. Rev Neurosci 7:29-46.

Deupree DL, Bradley J, Turner DA. 1993. Age-related alterations in potentiation in the CA1 region in F344 rats. Neurobiol Aging 14:249-258.

Dringenberg HC, Oliveira D, Habib D. 2008. Predator (cat hair)-induced enhancement of hippocampal long-term potentiation in rats: involvement of acetylcholine. Learn Mem 15:112116.

Dudar JD. 1977. The role of the septal nuclei in the release of acetyl-choline from the rabbit cerebral cortex and dorsal hippocampus and the effect of atropine. Brain Res 129:237-246.

Dudek SM, Bear MF (1992) Homosynaptic long-term depression in area CA1 of hippocampus and effects of N-methyl-D-aspartate receptor blockade. Proc Natl Acad Sci U S A 89:4363-4367.

Dunnett SB, Gage FH, Björklund A, Stenevi U, Low WC, Iversen SD. 1982. Hippocampal deafferentation: transplant-derived reinnervation and functional recovery. Scand J Psychol 1:104111. 
Dutar P, Bassant MH, Senut MC, Lamour Y. 1995. The septohippocampal pathway: structure and function of a central cholinergic system. Physiol Rev 75:393-427.

Eichenbaum H. 2006. Remembering: functional organization of the declarative memory system. Curr Biol 22:643-645.

Eichenbaum H. 1996. Learning from LTP: a comment on recent attempts to identify cellular and molecular mechanisms of memory. Learn and Mem 3:61-73.

Emptage NJ, Reid CA, Fine A, Bliss TV. 2003. Optical quantal analysis reveals a presynaptic component of LTP at hippocampal Schaffer-associational synapses. Neuron 38: 797-804.

Fisher R, Johnston D. 1990. Differential modulation of single voltage-gated calcium channels by cholinergic and adrenergic agonists in adult hippocampal neurons. J Neurophysiol 64:1291-1302.

Fischer W, Chen KS, Gage FH, Björklund A. 1992. Progressive decline in spatial learning and integrity of forebrain cholinergic neurons in rats during aging. Neurobiol Aging 13: 9-23.

Fischer W, Gage FH, Björklund A. 1989. Degenerative Changes in Forebrain Cholinergic Nuclei Correlate with Cognitive Impairments in Aged Rats. Eur J Neurosci 1:34-45.

Foster TC, McNaughton BL. 1991. Long-term enhancement of CA1 synaptic transmission is due to increased quantal size, not quantal content. Hippocampus 1:79-91.

Freund TF, Antal M. 1988. GABA-containing neurons in the septum control inhibitory interneurons in the hippocampus. Nature 336:170-173.

Frick KM, Baxter MG, Markowska AL, Olton DS, Price DL. 1995. Age-related spatial reference and working memory deficits assessed in the water maze. Neurobiol Aging 16: 149-160.

Frielingsdorf H, Thal LJ, Pizzo DP. 2006. The septohippocampal cholinergic system and spatial working memory in the Morris water maze. Behav Brain Res 168:37-46. 
from CA1 pyramidal cells in the guinea pig hippocampal slice. Neurosci Res Commun 6:149155.

Fujii S, Ji Z, Morita N, Sumikawa K. 1999. Acute and chronic nicotine exposure differentially facilitate the induction of LTP. Brain Res 846:137-143.

Fukunaga K, Muller D, Miyamoto E. 1995. Increased phosphorylation of Ca2+/calmodulindependent protein kinase II and its endogenous substrates in the induction of long-term potentiation. J Biol Chem 270:6119-6124.

Gage FH, Björklund A. 1986. Cholinergic septal grafts into the hippocampal formation improve spatial learning and memory in aged rats by an atropine-sensitive mechanism. J Neurosci 6: 28372847.

Gage FH, Björklund A, Stenevi U. 1984. Cells of origin of the ventral cholinergic septohippocampal pathway undergoing compensatory collateral sprouting following fimbria-fornix transection. Neurosci Lett 44: 211-216.

Gelinas JN, Banko JL, Peters MM, Klann E, Weeber EJ, Nguyen PV. 2008. Activation of exchange protein activated by cyclic-AMP enhances long-lasting synaptic potentiation in the hippocampus. Learn Mem 15:403-411.

Gray JA, McNaughton N. 1983. Comparison between the behavioural effects of septal and hippocampal lesions: a review. Neurosci Biobehav Rev 7:119-188.

Habib D, Dringenberg HC. Surprising similarity between mechanisms mediating low (1 Hz)-and high $(100 \mathrm{~Hz})$-induced long-lasting synaptic potentiation in CA1 of the intact hippocampus. Neuroscience, in press.

Habib D, Dringenberg HC. 2010. Low-frequency-induced synaptic potentiation: A paradigm shift in the field of memory-related plasticity mechanisms? Hippocampus 20: 29-35 
Habib D, Dringenberg HC. 2009. Alternating low frequency stimulation of medial septal and commissural fibers induces NMDA-dependent, long-lasting potentiation of hippocampal synapses in urethane- anesthetized rats. Hippocampus 19: 299-307.

Hager AM, Dringenberg HC. Training-induced plasticity in the visual cortex following visual discrimination learning. Learn Mem, in press.

Hasselmo ME, Giocomo LM. 2006. Cholinergic modulation of cortical function. J Mol Neurosci $30: 133-135$.

Hasselmo ME. 1999. Neuromodulation: acetylcholine and memory consolidation. Trends Cogn Sci 3: 351-359.

He Y, Janssen WG, Morrison JH. 1998. Synaptic coexistence of AMPA and NMDA receptors in the rat hippocampus: a postembedding immunogold study. J Neurosci Res 54:444-449.

Hebb, DO. 1949. The Organization of Behavior. New York: John Wiley.

Heynen AJ, Bear MF. 2001. Long-term potentiation of thalamocortical transmission in the adult visual cortex in vivo. J Neurosci 21:9801-9813.

Hoffman KL, Battaglia FP, Harris K, MacLean JN, Marshall L, Mehta MR. 2007. The upshot of up states in the neocortex: from slow oscillations to memory formation. J Neurosci 27:11838-11841.

Hölscher, C. 1999. Synaptic plasticity and learning and memory: LTP and beyond. J Neurosci Res $58: 62-75$.

Hölscher C. 1997. Long-term potentiation: a good model for learning and memory? Prog Neuropsychopharmacol Biol Psychiatry 21:47-68.

Hornung OP, Danker-Hopfe H, Heuser I. 2005. Age-related changes in sleep and memory: commonalities and interrelationships. Exp Gerontol 40:279-285. 
Houser CR, Crawford GD, Barber RP, Salvaterra PM, Vaughn JE. 1983. Organization and morphological characteristics of cholinergic neurons: an immunocytochemical study with a

Huang YY, Kandel ER. 2007. Low-frequency stimulation induces a pathway-specific late phase of LTP in the amygdala that is mediated by PKA and dependent on protein synthesis. Learn Mem 14:497-503.

Huang YY, Kandel ER. 2006. Age-related enhancement of a protein synthesis-dependent late phase of LTP induced by low frequency paired-pulse stimulation in hippocampus. Learn Mem 13:298306monoclonal antibody to choline acetyltransferase. Brain Res 266:97-119.

Huang YY, Martin KC, Kandel ER. 2000. Both protein kinase A and mitogen-activated protein kinase are required in the amygdala for the macromolecular synthesis-dependent late phase of long-term potentiation. J Neurosci 20:6317-6325.

Huang YY, Kandel ER. 1998. Postsynaptic induction and PKA-dependent expression of LTP in the lateral amygdala. Neuron 21:169-178.

Huber R, Ghilardi MF, Massimini M, Tononi G. 2004. Local sleep and learning. Nature 430:78-81.

Huerta PT, Lisman JE. 1993. Heightened synaptic plasticity of hippocampal CA1 neurons during a cholinergically induced rhythmic state. Nature 364:723-725.

Hyman JM, Wyble BP, Goyal V, Rossi CA, Hasselmo ME. 2003. Stimulation in hippocampal region CA1 in behaving rats yields long-term potentiation when delivered to the peak of theta and longterm depression when delivered to the trough. J Neurosci 23:11725-11731.

Impey S, Obrietan K, Wong ST, Poser S, Yano S, Wayman G, Deloulme JC, Chan G, Storm DR. 1998. Cross talk between ERK and PKA is required for Ca2+ stimulation of CREB-dependent transcription and ERK nuclear translocation. Neuron 21:869-883. 
Izquierdo I, Medina JH. 1995. Correlation between the pharmacology of long-term potentiation and the pharmacology of memory. Neurobiol Learn Mem. 63:19-32.

Jaffe D, Johnston D. 1990. Induction of long-term potentiation at hippocampal mossy-fiber synapses follows a Hebbian rule. J Neurophysiol 64:948-960.

Jarrard LE. 1993. On the role of the hippocampus in learning and memory in the rat. Behav Neural Biol 60:9-26.

Jay TM, Burette F, Laroche S. 1996. Plasticity of the hippocampal-prefrontal cortex synapses. J Physiol Paris 90:361-366.

Ji D, Lape R, Dani JA. 2001. Timing and location of nicotinic activity enhances or depresses hippocampal synaptic plasticity. Neuron 31:131-141.

Kandel ER, Squire LR. 2000. Neuroscience: Breaking down scientific barriers to the study of brain and mind. Science 290:1113-1120.

Katz B, Miledi R. 1968. The role of calcium in neuromuscular facilitation. J Physiol 195:481-492.

Kelsey JE, Landry BA. 1988. Medial septal lesions disrupt spatial mapping ability in rats. Behav Neurosci 102:289-293.

Kirkwood A, Rioult MG, Bear MF. 1996. Experience-dependent modification of synaptic plasticity in visual cortex. Nature 381:526-528.

Kleschevnikov AM, Routtenberg A. 2001. PKC activation rescues LTP from NMDA receptor blockade. Hippocampus 11:168-175.

Köhler C, Chan-Palay V, Wu JY. 1984. Septal neurons containing glutamic acid decarboxylase immunoreactivity project to the hippocampal region in the rat brain. Anat Embryol (Berl) $169: 41-44$.

Krnjević K, Ropert N, Casullo J. 1988. Septohippocampal disinhibition. Brain Res 438:182-192. 
Krnjević K, Ropert N. 1982. Electrophysiological and pharmacological characteristics of facilitation of hippocampal population spikes by stimulation of the medial septum. Neuroscience 7:21652183.

Kudrimoti HS, Barnes CA, McNaughton BL. 1999. Reactivation of hippocampal cell assemblies: effects of behavioral state, experience, and EEG dynamics. J Neurosci 19:4090-4101.

Lanté F, Cavalier M, Cohen-Solal C, Guiramand J, Vignes M. 2006b. Developmental switch from LTD to LTP in low frequency-induced plasticity. Hippocampus 16:981-989.

Lanté F, de Jésus Ferreira MC, Guiramand J, Récasens M, Vignes M. 2006a. Low-frequency stimulation induces a new form of LTP, metabrotropic glutamate (mGlu5) receptor-and PKAdependent, in the CA1 area of the rat hippocampus. Hippocampus 16:345-360.

Larson J, Lynch G. 1988. Role of N-methyl-D-aspartate receptors in the induction of synaptic potentiation by burst stimulation patterned after the hippocampal theta-rhythm. Brain Res 441:111-118.

Larson J, Wong D, Lynch G. 1986. Pattered stimulation at the theta frequency is optimal for the induction of hippocampal long-term potentiation. Brain Res 368:347-350.

Lauri SE, Vesikansa A, Segerstråle M, Collingridge GL, Isaac JT, Taira T. 2006. Functional maturation of CA1 synapses involves activity-dependent loss of tonic kainate receptor-mediated inhibition of glutamate release. Neuron 50:415-429.

Léránth C, Frotscher M. 1987. Cholinergic innervation of hippocampal GAD- and somatostatinimmunoreactive commissural neurons. J Comp Neurol 261:33-47.

Leung LS, Shen B, Rajakumar N, Ma J. 2003. Cholinergic activity enhances hippocampal long-term potentiation in CA1 during walking in rats. J Neurosci 23:9297-9304. 
Li H, Chen A, Xing G, Wei M-L, Rogawski MA. 2001. Kainate receptor-mediated heterosynaptic facilitation in the amygdala. Nat Neurosci 4:612-620.

Li H, Weiss S. R, Chuang D. M, Post RM, Rogawski MA. 1998. Bidirectional synaptic plasticity in the rat basolateral amygdala: characterization of an activity-dependent switch sensitive to the presynaptic metabotropic glutamate receptor antagonist 2S- $\alpha$ ethylglutamic acid. J Neurosci. 18:1662-1670.

Lisman J, Schulman H, Cline H. 2002. The molecular basis of CaMKII function in synaptic and behavioural memory. Nat Rev Neurosci 3:175-190.

Lissin DV, Gomperts SN, Carroll RC, Christine CW, Kalman D, Kitamura M, Hardy S, Nicoll RA, Malenka RC, von Zastrow M. 1998. Activity differentially regulates the surface expression of synaptic AMPA and NMDA glutamate receptors. Proc Natl Acad Sci USA 95:7097-7102.

Lovinger DM. 2010. Neurotransmitter roles in synaptic modulation, plasticity and learning in the dorsal striatum. Neuropharmacology 58:951-961.

Low WC, Lewis PR, Bunch ST, Dunnett SB, Thomas SR, Iversen SD, Björklund A, Stenevi U. 1982. Function recovery following neural transplantation of embryonic septal nuclei in adult rats with septohippocampal lesions. Nature 300:260-262.

Luscher C, Nicoll RA, Malenka RL, Muller D. 2000. Synaptic plasticity and dynamic modulation of the postsynaptic membrane. Nature Neurosci 3:545-550.

Lynch G, Larson J, Kelso S, Barrionuevo G, Schottler F. 1983. Intracellular injections of EGTA block induction of hippocampal long-term potentiation. Nature 305:719-721.

Lynch MA. 2004. Long-term potentiation and memory. Physiol Rev 84:87-136.

Magnusson KR. 1995. Differential effects of aging on binding sites of the activated NMDA receptor complex in mice. Mech Ageing Dev 84:227-243. 
Magnusson KR. 1998. Aging of glutamate receptors: correlations between binding and spatial memory performance in mice. Mech Ageing Dev 104:227-248.

Malenka RC, Bear MF. 2004. LTP and LTD: an embarrassment of riches. Neuron 44:5-21.

Malenka RC, Nicoll RA. 1999. Long-term-potentiation, a decade of progress? Science 285: 18701874.

Malenka RC, Kauer JA, Zucker RS, Nicoll RA. 1988. Postsynaptic calcium is sufficient for potentiation of hippocampal synaptic transmission. Science 242:81-84.

Malinow R, Tsien RW. 1990. Presynaptic enhancement shown by whole-cell recordings of long-term potentiation in hippocampal slices. Nature 346:177-180.

Manseau F, Danik M, Williams S. 2005. A functional glutamatergic neurone network in the medial septum and diagonal band area. J Physiol 566:865-884.

Markevich V, Scorsa AM, Dawe GS, Stephenson JD. 1997. Cholinergic facilitation and inhibition of long-term potentiation of CA1 in the urethane-anaesthetized rats. Brain Res 754:95-102.

Markram H, Segal M. 1990. Long-lasting facilitation of excitatory postsynaptic potentials in the rat hippocampus by acetylcholine. J Physiol 427:381-393.

Marshall L, Helgadóttir H, Mölle M, Born J. 2006. Boosting slow oscillations during sleep potentiates memory. Nature 444:610-613.

Martin SJ, Clark RE. 2007. The rodent hippocampus and spatial memory: from synapses to systems. Cell Mol Life Sci 64:401-431.

Martin SJ, Morris, RG. 2002. New life in an old idea: the synaptic plasticity and memory hypothesis revisited. Hippocampus 12: 609-636.

Martin SJ, Grimwood PD, Morris RGM. 2000. Synaptic plasticity and memory: An evaluation of the hypothesis. Annu Rev Neurosci 23:649-711. 
Maruki K, Izaki Y, Nomura M, Yamauchi T. 2001. Differences in paired-pulse facilitation and longterm potentiation between dorsal and ventral CA1 regions in anesthetized rats. Hippocampus 11:655-661.

Massey PV, Bashir ZI. 2007. Long-term depression: multiple forms and implications for brain function. Trends Neurosci 30:176-184.

Matsuyama S, Matsumoto A, Enomoto T, Nishizaki T. 2000. Activation of nicotinic acetylcholine receptors induces long-term potentiation in vivo in the intact mouse dentate gyrus. Eur J Neurosci $12: 3741-3747$.

Mayford M, Wang J, Kandel ER, O’Dell TJ. 1995. CaMKII regulates the frequency-response function of hippocampal synapses for the production of both LTD and LTP. Cell 81: 891-904.

McDonald RJ, Hong NS, Craig LA, Holahan MR, Louis M, Muller RU. 2005. NMDA-receptor blockade by CPP impairs post-training consolidation of a rapidly acquired spatial representation in rat hippocampus. Eur J Neurosci 22:1201-1213.

McDonald RJ, White NM. 1994. Parallel information processing in the water maze: Evidence for independent memory systems involving dorsal striatum and hippocampus. Behav Neural Biol $61: 260-270$.

McDonald RJ, White NM. 1993. A triple dissociation of memory systems: hippocampus, amygdala, and dorsal striatum. Behav Neurosci 107:3-22.

McGaugh JL. 2000. Memory-a century of consolidation. Science 287:248-251.

McNaughton BL. 1983. Activity-dependent modulation of hippocampal synaptic efficacy: Some implications for memory processes. In Neurobiology of the hippocampus, ed. W Siefert, p. 233252. London: Academic. 
Medina JH, Izquierdo I. 1995. Retrograde messengers, long-term potentiation and memory. Brain Res Brain Res Rev 21:185-194.

Milner TA, Amaral DG. 1984. Evidence for a ventral septal projection to the hippocampal formation of the rat. Exp Brain Res 55:579-585.

Miyamoto E. 2006. Molecular mechanism of neuronal plasticity: induction and maintenance of longterm potentiation in the hippocampus. J Pharmacol Sci 100:433-442.

Moore CI, Browning MD, Rose GM. 1993. Hippocampal plasticity induced by primed burst, but not long-term potentiation, stimulation is impaired in area CA1 of aged Fischer 344 rats. Hippocampus 3:57-66.

Morris RG. 1986. Allocentric spatial learning by hippocampectomised rats: a further test of the "spatial mapping" and "working memory" theories of hippocampal function. Q J Exp Pschol B $38: 365-395$.

Morris RG, Garrud P, Rawlins JN, O'Keefe J. 1982. Place navigation impaired in rats with hippocampal lesions. Nature 297:681-683.

Morris, RG. 1981. Spatial localization does not require the presence of local cues. Learn Motiv $12: 239-261$.

Nicoll RA, Schmitz D. 2005. Synaptic plasticity at hippocampal mossy fibre synapses. Nat Rev Neurosci 6:863-876.

Nicoll RA, Malenka RC. 1999. Expression mechanisms underlying NMDA receptor-dependent longterm potentiation. Ann N Y Acad Sci 868:515-525.

Nilsson OG, Gage FH. 1993. Anticholinergic sensitivity in the aging rat septohippocampal system as assessed in a spatial memory task. Neurobiol Aging 14:487-497. 
Okulski P, Hess G, Kaczmarek L. 2002. Anisomycin treatment paradigm affects duration of longterm potentiation in slices of the amygdala. Neurosci 114:1-5.

Oldford E, Castro-Alamancos MA. 2003. Input-specific effects of acetylcholine on sensory and intracortical evoked responses in the 'barrel cortex' in vivo. Neuroscience 117:769-778.

Ovsepian SV. 2006. Enhancement of the synchronized firing of CA1 pyramidal cells by medial septum preconditioning: time-dependent involvement of muscarinic cholinoceptors and GABAB receptors. Neurosci Lett 393:1-6.

Ovsepian SV, Anwyl R, Rowan MJ. 2004. Endogenous acetylcholine lowers the threshold for longterm potentiation induction in the CA1 area through muscarinic receptor activation: in vivo study. Eur J Neurosci 20:1267-1275.

Packard MG, Cahill L, McGaugh JL. 1994. Amygdala modulation of hippocampal-dependent and caudate nucleus-dependent memory processes. Proc Natl Acad Sci U S A 91:8477-8481.

Paxinos G, Watson C. 1998. The Rat Brain in Stereotaxic Coordinates, $4^{\text {th }}$ Edn. Academic Press, San Diego.

Peigneux P, Laureys S, Fuchs S, Collette F, Perrin F, Reggers J, Phillips C, Degueldre C, Del Fiore G, Aerts J, Luxen A, Maquet P. 2004. Are spatial memories strengthened in the human hippocampus during slow wave sleep? Neuron 44:535-545.

Pennartz CM, Berke JD, Graybiel AM, Ito R, Lansink CS, van der Meer M, Redish AD, Smith KS, Voorn P. 2009. Corticostriatal Interactions during Learning, Memory Processing, and Decision Making. J Neurosci 29:12831-12838.

Popescu AT, Saghyan AA, Nagy FZ, Pare D.Facilitation of corticostriatal plasticity by the amygdala requires $\mathrm{Ca}^{2+}$-induced $\mathrm{Ca}^{2+}$ release in the ventral striatum. J Neurophysiol, in press. 
Popescu AT, Saghyan AA, Paré, D. 2007. NMDA-dependent facilitation of corticostriatal plasticity by the amygdala. Proc Natl Acad Sci U S A 104:341-346.

potentiation in the amygdala. Nature 390: 552-553.

Raymond CR. 2007. LTP forms 1, 2 and 3: different mechanisms for the "long" in long-term potentiation. Trends Neurosci 30:167-175.

Riedel G, Platt B, Micheau J. 2003. Glutamate receptor function in learning and memory. Behav Brain Res 140:1-47.

Riedel G, Seidenbechera T, Reymanna KG. 1994. LTP in hippocampal CA1 of urethane-narcotized rats requires stronger tetanization parameters. Physiol Behav 55:1141-1146.

Riekkinen P Jr, Sirviö J, Aaltonen M, Riekkinen P. 1990. Effects of concurrent manipulations of nicotinic and muscarinic receptors on spatial and passive avoidance learning. Pharmacol Biochem Behav 37:405-410.

Rioult-Pedotti MS, Friedman D, Hess G, Donoghue JP. 1998. Strengthening of horizontal cortical connections following skill learning. Nat Neurosci 1:230-234.

Roberson ED, English JD, Adams JP, Selcher JC, Kondratick C, Sweatt JD. 1999. The mitogenactivated protein kinase cascade couples PKA and PKC to cAMP response element binding protein phosphorylation in area CA1 of hippocampus. J Neurosci 19:4337-4348.

Roesler R, Reolon GK, Luft T, Martins MR, Schröder N, Vianna MR, Quevedo J. 2005. NMDA receptors mediate consolidation of contextual memory in the hippocampus after context preexposure. Neurochem Res 30:1407-1411.

Rogan MT, Staubli UV, LeDoux JE. 1997. Fear conditioning induces associative long-term in the amygdala. Nature 390: 604-607. 
Rogan MT, LeDoux JE. 1995. LTP is accompanied by commensurate enhancement of auditoryevoked responses in a fear conditioning circuit. Neuron 15:127-136.

Rosenzweig ES, Barnes CA. 2003. Impact of aging on hippocampal function: Plasticity, network dynamics, and cognition. Prog in Neurobiol 69:143-179.

Rosenzweig ES, Rao G, McNaughton BL, Barnes CA. 1997. Role of temporal summation in agerelated LTP-induction deficits. Hippocampus 7: 549-558.

Schall KP, Kerber J, Dickson CT. 2008. Rhythmic constraints on hippocampal processing: state and phase-related fluctuations of synaptic excitability during theta and the slow oscillation. $\mathrm{J}$ Neurophysiol 99:888-899.

Schulz PE. 1997. Long-term potentiation involves increases in the probability of neurotransmitter release. Proc Natl Acad Sci U S A 94:5888-5893.

Schulz PE, Fitzgibbons JC. 1997. Differing mechanisms of expression for short- and long-term potentiation. J Neurophysiol 78:321-334.

Schulz PE, Cook EP, Johnston D. 1995. Using paired-pulse facilitation to probe the mechanisms for long-term potentiation (LTP). J Physiol Paris 89:3-9.

Schulz PE, Cook EP, Johnston D. 1994. Changes in paired-pulse facilitation suggest presynaptic involvement in long-term potentiation. J Neurosci 14:5325-5337.

Segal M, Greenberger V, Pearl E. 1989. Septal transplants ameliorate spatial deficits and restore cholinergic functions in rats with a damaged septo-hippocampal connection. Brain Res 500:139148.

Selcher JC, Weeber EJ, Christian J, Nekrasova T, Landreth GE, Sweatt JD. 2003. A role for ERK MAP kinase in physiologic temporal integration in hippocampal area CA1. Learn Mem 10:26-39. 
Shankar S, Teyler TJ, Robbins N. 1998. Aging differentially alters forms of long-term potentiation in rat hippocampal area CA1. J Neurophysiol 79:334-341.

Shen J, Barnes CA, McNaughton BL, Skaggs WE, Weaver KL. 1997. The effect of aging on experience-dependent plasticity of hippocampal place cells. J Neurosci 17:6769-6782.

Siapas AG, Wilson MA. 1998. Coordinated interactions between hippocampal ripples and cortical spindles during slow-wave sleep. Neuron 21:1123-1128.

Sirota A, Buzsáki G. 2005. Interaction between neocortical and hippocampal networks via slow oscillations. Thalamus Relat Syst 3:245-259.

Sotty F, Danik M, Manseau F, Laplante F, Quirion R, Williams S. 2003. Distinct electrophysiological properties of glutamatergic, cholinergic and GABAergic rat septohippocampal neurons: novel implications for hippocampal rhythmicity. J Physiol 551:927-943.

Speechley WJ, Hogsden JL, Dringenberg HC. 2007. Continuous white noise exposure during and after auditory critical period differentially alters bidirectional thalamocortical plasticity in rat auditory cortex in vivo. Eur J Neurosci 26:2576-2584.

Squire LR. 1992. Memory and the hippocampus: a synthesis from findings with rats, monkeys, and humans. Psychol Rev 99:195-231.

Stäubli U, Scafidi J. 1999. Time-dependent reversal of long-term potentiation in area CA1 of the freely moving rat induced by theta pulse stimulation. J Neurosci 19:8712-8719.

Stäubli U, Lynch G. 1987. Stable hippocampal long-term potentiation elicited by 'theta' pattern stimulation. Brain Res 435:227-234.

Steriade M, Contreras D, Curró Dossi R, Nuñez A. 1993. The slow $(<1 \mathrm{~Hz})$ oscillation in reticular thalamic and thalamocortical neurons: scenario of sleep rhythm generation in interacting thalamic and neocortical networks. J Neurosci 13:3284-3299. 
Straube T, Frey JU. 2003. Involvement of beta-adrenergic receptors in protein synthesis-dependent late long-term potentiation (LTP) in the dentate gyrus of freely moving rats: the critical role of the LTP induction strength. Neuroscience 119:473-479.

Sutherland RJ, Whishaw IQ, Kolb B. 1983. A behavioural analysis of spatial localization following electrolytic, kainate- or colchicine-induced damage to the hippocampal formation in the rat. Behav Brain Res 7:133-153.

Teyler TJ, Cavus I, Coussens C, DiScenna P, Grover L, Lee YP, Little Z. 1994. Multideterminant role of calcium in hippocampal synaptic plasticity. Hippocampus 4: 623-634.

Thiels E, Barrionuevo G, Berger TW. 1994. Excitatory stimulation during postsynaptic inhibition induces long-term depression in hippocampus in vivo. J Neurophysiol 72: 3009-3016.

Thomas MJ, Moody TD, Makhinson M, O’Dell TJ. 1996. Activity dependent beta-adrenergic modulation of low frequency stimulation induced LTP in the hippocampal CA1 region. Neuron $17: 475-482$.

Tielen AM, Mollevanger WJ, Lopes da Silva FH, Hollander CF. 1983. Neuronal plasticity in hippocampal slices of extremely old rats. In: Gispen WH., Traber J. (Eds), Aging of the Brain. Elsevier, Amsterdam, pp. 73-84.

Tóth K, Borhegyi Z, Freund TF. 1993. Postsynaptic targets of GABAergic hippocampal neurons in the medial septum-diagonal band of broca complex. J Neuroci 13:3712-3724.

Van Gool WA, Mirmiran M. 1983. Age-related changes in the sleep pattern of male adult rats. Brain Res 279:384-398.

Vanderwolf CH. 1988. Cerebral activity and behavior: control by central cholinergic and serotonergic systems. Int Rev Neurobiol 30:225-340. 
Vanderwolf CH. 1969. Hippocampal electrical activity and voluntary movement in the rat.

Electroencephalogr Clin Neurophysiol 26:407-418.

Voronin LL, Kuhnt U. 1990. Long-term potentiation affects facilitation ratio of EPSPs recorded from CA1 pyramidal cells in the guinea-pig hippocampal slice. Neurosci Res Commun 6:149-155.

Wainer BH, Bolam JP, Freund TF, Henderson Z, Totterdell S, Smith AD. 1984. Cholinergic synapses in the rat brain: a correlated light and electron microscopic immunohistochemical study employing a monoclonal antibody against choline acetyltransferase. Brain Res 308:69-76.

Wasling P, Hanse E, Gustafsson B. 2002. Long-term depression in the developing hippocampus: low induction threshold and synapse nonspecificity. J Neurosci 22:1823-1830.

Whitlock JR, Heynen AJ, Shuler MG, Bear MF. 2006. Learning induces long-term potentiation in the hippocampus. Science 13:1093-1097.

Williams JH, Errington ML, Lynch MA, Bliss TV. 1989. Arachidonic acid induces a long-term activity-dependent enhancement of synaptic transmission in the hippocampus. Nature 341:739742.

Wolansky T, Clement EA, Peters SR, Palczak MA, Dickson CT. 2006. Hippocampal slow oscillation: a novel EEG state and its coordination with ongoing neocortical activity. J Neurosci $26: 6213-6229$.

Yao H, Dan Y. 2005. Synaptic learning rules, cortical circuits, and visual function. Neuroscientist 11:206-216. 